\title{
THE LOW DENSITY LIMIT IN FINITE TEMPERATURE CASE
}

\author{
L. ACCARDI AND Y. G. LU*
}

\section{§ 1. Introduction}

The low density limit in the Boson Fock case has been investigated in [1] where also the physical meaning and their motivations have been explained (cf. also [0]). From these papers one knows how the number processes can be obtained from a quantum Hamiltonian model via a certain limit procedure.

But the physically more meaningful case is the one in which the initial state of the reservoir is a finite temperature rather than Fock state. The present paper is devoted to do this case.

In the present paper, our physical model and essential assumptions are same as ones of [1] (except the Fock state). We consider a "System + Reservoir" model described by a system Hilbert space $H_{0}$ and a (one particle) reservoir Hilbert space $H_{1}$; the system Hamiltonian $H_{s}$; the (one particle) reservoir Hamiltonian $\Delta$, and the associated one particle reservoir evolution

$$
S_{t}^{0}:=\exp (-i t \Delta)
$$

which is a one parameter unitary group on $B\left(H_{1}\right)$.

Let be given, on the CCR $C^{*}$-algebra over $H_{1}$ (cf. [11]), a gauge invariant quasi-free state $\varphi^{(z)}$ with fugacity $z^{2}$, i.e. for each $f \in H_{1}$,

$$
\varphi^{(z)}(W(f))=\exp \left(-\frac{1}{2}\left\langle f,\left(1+z^{2} e^{-\frac{1}{2} H \beta}\right)\left(1-z^{2} e^{-\frac{1}{2} H \beta}\right)^{-1} f\right\rangle\right)
$$

where $H$ is a self-adjoint operator which, will be supposed in this paper, commutes with $\Delta$ and $W(f)$ is the Weyl operator with test function $f$. Moreover up to GNS-construction one can write the left hand side of (1.1) as

$$
\left\langle\Phi_{z}, W(f) \Phi_{z}\right\rangle
$$

Received March 29, 1991.

* On leave of absence from Beijing Normal University 
where the Weyl operators act on a Hilbert space $\mathscr{H}_{z}$ and $\Phi_{z}$ is a cyclic vector for the Weyl algebra. For any Hilbert space $\mathscr{H}$, we shall denote $\Gamma(\mathscr{H})$ the Fock space over $\mathscr{H}$.

As in [1], we consider the situation in which the interaction between the System and Reservoir is of the form

$$
V:=i\left(D \otimes A^{+}\left(g_{0}\right) A\left(g_{1}\right)-D^{+} \otimes A\left(g_{1}\right)^{+} A\left(g_{0}\right)\right)
$$

and the time evolution in the interaction picture is defined by

$$
U_{t}:=e^{i t H \mathrm{fr}} \cdot e^{-i t H \mathrm{tot}}
$$

where

$$
H_{\mathrm{fr}}:=H_{0} \otimes 1+1 \otimes d \boldsymbol{\Gamma}(-\Delta)
$$

is called the free Hamiltonian and

$$
H_{\text {tot }}:=H_{\mathrm{fr}}+V
$$

the total Hamiltonian.

A simple computation shows that the time evolution $U(t)$ is the solution of the following ordinary differential equation

$$
\frac{d}{d t} U_{t}=i V(t) \cdot U_{t}
$$

and where

$$
V(t):=e^{i t H \mathrm{fr}} V e^{-i t H \mathrm{fr}}
$$

Now, as in [1], let us suppose that

1) $g_{0}$ and $g_{1}$ have disjoint spectrum sets, i.e.

$$
\left\langle g_{0}, S_{t}^{0} \quad g_{1}\right\rangle=0, \quad \forall t \in \mathbf{R}
$$

2) the rotating wave approximation:

$$
e^{i t H_{0}} D e^{-i t H_{0}}=e^{-i t\left(\omega_{0}-\omega_{1}\right)} D, \quad \text { for some } \omega_{0} \neq \omega_{1} .
$$

These two conditions have rather good physical explanations and under them, the same arguments as in [1] (or [0]) imply that (1.6) can be rewritten as

$$
V(t)=i\left(D \otimes A^{+}\left(S_{t} g_{0}\right) A\left(S_{t} g_{0}\right)-D^{+} \otimes A\left(S_{t} g_{1}\right)^{+} A\left(S_{t} g_{0}\right)\right)
$$

where $S_{t}$ is a unitary group satisfying

$$
S_{t} g_{0}=S_{t}^{0} e^{-i t \omega_{0}} g_{0} \quad S_{t} g_{1}=S_{t}^{0} e^{i t \omega_{1}} g_{1} .
$$


In this paper we shall directly start our research from (1.9) and in certain sense forget the motivation of this assumption, i.e. starting from (1.9), we investigate, purely mathematically, the low density limit. Of course one of physically meaningful case of our study is the one with the conditions (1.7) and (1.8). Moreover, we shall suppose that the unitary group $\left\{S_{t}\right\}_{t \in \mathbf{R}}$ commutes with $\Delta$; there exists a nonzero subset $K$ of $H_{1}$ such that $g_{0}, g_{1} \in K$,

$$
\int_{\mathbf{R}}\left|\left\langle f, S_{t} g\right\rangle\right| d t<\infty, \forall f, g \in K
$$

and for each $f, g \in K$, the series

$$
\sum_{n=1}^{\infty} z^{n} \cdot \int_{\mathbf{R}}\left|\left\langle f, S_{t} \exp (-\beta n H) g\right\rangle\right| d t
$$

has a positive convergence radius.

With the condition (1.11), one can define a Hilbert space $\{K,(\cdot \mid \cdot)\}$ in same way as [1], where, for each $f, g \in K$,

$$
(f \mid g):=\int_{\mathbf{R}}\left\langle f, S_{t} g\right\rangle d t
$$

Inspired by [1], in this paper we shall investigate the limit

$$
\lim _{z \rightarrow 0}\left\langle u \otimes W\left(z \int_{S / z^{2}}^{T / z^{2}} S_{u} f d u\right) \Phi_{z}, U_{t / z^{2 v}} \otimes W\left(z \int_{S^{\prime} / z^{2}}^{T^{\prime} / z^{2}} S_{u} f^{\prime} d u\right) \Phi_{z}\right\rangle
$$

It will be proved that the limit (1.13) exists and is equal to

$$
\left\langle u \otimes W\left(\chi_{[S, T]} \otimes f\right) \Psi, U(t) v \otimes W\left(\chi_{\left[S^{\prime}, T^{\prime}\right]} \otimes f^{\prime}\right) \Psi\right\rangle
$$

where the scalar product in (1.14) is meant in a Fock quantum Brownian motion in the sense of [1] (cf. also [0]) and $U(t)$ is a Markovian cocycle satisfying a quantum stochastical differential equation (q.s.d.e.). More precisely, the main result in the present paper is the following:

TheOREm (1.1). For each $f, f^{\prime}, g_{0}, g_{1} \in K, u, v \in H_{0}, D \in B\left(H_{0}\right), S, T, S^{\prime}$, $T^{\prime} \in \mathbf{R}, t \geq 0$, if $t, g_{0}, g_{1}$ satisfy the condition

$$
t<\frac{1}{16\|D\|} ;\|g\|_{s}^{2}:=\left(\max _{g, g^{\prime} \in\left\{g, g_{1}, e^{\left.-\frac{1}{2} \beta H_{g 0}, e^{-\frac{1}{2} \beta H} g_{1}\right\}}\right.} \int_{-\infty}^{\infty}\left|\left\langle g, S_{t} g^{\prime}\right\rangle\right| d t\right)^{2}<\frac{1}{16\|D\|}
$$

then the low density limit (1.13) exists and is equal to (1.14) where, $U(t)$ is the solution of q.s.d.e.

$$
\begin{gathered}
U(t)=1+\sum_{\varepsilon \in\{0,1\}} \int_{0}^{t}\left[D_{1}(\varepsilon) \otimes d N_{s}\left(g_{\varepsilon}, g_{1-\varepsilon}\right)\right. \\
\left.+D_{2}(\varepsilon) \otimes d N_{s}\left(g_{\varepsilon}, g_{\varepsilon}\right)+\left(D_{3}(\varepsilon)+D_{\varepsilon}\left\langle g_{1-\varepsilon}, e^{-\frac{1}{2} \beta H} g_{\varepsilon}\right\rangle\right) \otimes 1 d s\right] U(s)
\end{gathered}
$$


on $H_{0} \otimes \Gamma\left(L^{2}(\mathbf{R}) \otimes(K,(\cdot \mid \cdot))\right)$ and

(i) $\Psi$ is the vacuum of $\Gamma\left(L^{2}(\mathbf{R}) \otimes(K,(\cdot \mid \cdot))\right)$;

(ii) $N_{s}\left(g, g^{\prime}\right):=N\left(\chi_{[0, s]} \otimes|g\rangle\left\langle g^{\prime}\right|\right)$;

(iii) For any operator $T$ on $L^{2}(\mathbf{R}) \otimes K, N(T)$ denotes the number operator generated by $T$ characterized by the condition

$$
e^{i t N(T)} W(\xi) \Psi=W\left(e^{i t T} \xi\right) \Psi, \forall \xi \in L^{2}(\mathbf{R}) \otimes K
$$

for $T$ self-adjoint, and extended by complex linearity to arbitrary $T$.

(iv) $D_{1}(\varepsilon) D_{2}(\varepsilon)$ and $D_{3}(\varepsilon)$ are given by (6.80), (6.81) and (6.65) respectively.

Remark. Since in the Hilbert space $\Gamma\left(L^{2}(\mathbf{R}) \otimes K\right)$, the set $\{W(\chi \otimes f) \Psi$; $\left.\chi \in L^{2}(\mathbf{R}), f \in K\right\}$ is a total subset, we know that the linear functions

$$
\left\langle u \otimes W\left(\chi_{[S, T]} \otimes f\right) \Psi, v \otimes W\left(\chi_{\left[S^{\prime}, T^{\prime}\right]} \otimes f^{\prime}\right) \Psi\right\rangle
$$

can separate (bounded) operators. Therefore the Theorem (1.1) gives a stochastic process as the low density limit of the time evolution of the original Hamiltonian model.

\section{§2. Preliminaries}

In this section, some preparations will be done for the following sections.

Lemma (2.1). For each $f, f^{\prime} \in K, S, S^{\prime}, T, T^{\prime} \in \mathbf{R}$,

$$
\begin{aligned}
& \lim _{z \rightarrow 0}\left\langle z \int_{S / z^{2}}^{T / z^{2}} S_{u} f d u, z \int_{S^{\prime} / z^{2}}^{T^{\prime} / z^{2}} S_{u} f^{\prime} d u\right\rangle
\end{aligned}
$$

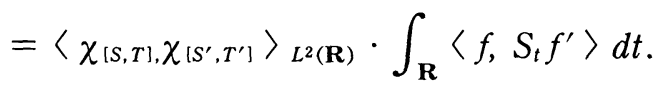

One can find the proof in [1].

Lemma (2.2). For each $n \in \mathbf{N},\left\{f_{k}\right\}_{k=1}^{n} \subset K,\left\{S_{k}, T_{k}\right\}_{k=1}^{n} \subset \mathbf{R},\left\{x_{k}\right\}_{k=1}^{n} \subset \mathbf{R}$,

$$
\begin{aligned}
\lim _{z \rightarrow 0}\left\langle\Phi_{z}, W\left(x_{1} z \int_{S_{1} / z^{2}}^{T_{1 / z^{2}}} S_{u} f_{1} d u\right) \cdots W\left(x_{n} z \int_{S_{n} / z^{2}}^{T_{n} / z^{2}} S_{u} f_{n} d u\right) \Phi_{z}\right\rangle \\
=\left\langle\Psi, W\left(x_{1} \chi_{\left[S_{1}, T_{1}\right]} \otimes f_{1}\right) \cdots W\left(x_{n} \chi_{\left[S_{n}, T n\right]} \otimes f_{n}\right) \Psi\right\rangle
\end{aligned}
$$

and the convergence is uniform for $\left\{x_{k}, S_{k}, T_{k}\right\}_{k=1}^{n}$ in a bounded set of $\mathbf{R}$, where, $\Psi$ is the vaccum of $\Gamma\left(L^{2}(\mathbf{R}) \otimes(K,(\cdot \mid \cdot))\right)$.

One can find the proof in [1]. 
Denote $H_{1}^{\iota}$ the conjugate Hilbert space of $H_{1}$, i.e.

$$
\begin{gathered}
\iota: H_{1} \longrightarrow H_{1}, \iota(\lambda f):=\bar{\lambda}_{\iota}(f) \\
\langle\iota(f), \iota(g)\rangle_{\iota}:=\langle g, f\rangle
\end{gathered}
$$

then, $H_{1}^{\iota}$ is a Hilbert space. It is well known that up to a unitary isomorphism

$$
\begin{gathered}
\Gamma\left(H_{1}\right)=\Gamma\left(H_{1}\right) \otimes \Gamma\left(H_{1}\right) \\
W(f)=W\left(Q_{+} f\right) \otimes W\left(Q_{-} f\right)
\end{gathered}
$$

for each $f \in H_{1}$ and

$$
\Phi_{z}=\Phi \otimes \Phi^{\iota}
$$

where $\Phi, \Phi^{\iota}$ are the vaccum vectors in $\Gamma\left(H_{1}\right)$ and $\Gamma\left(H_{1}^{\iota}\right)$ respectively and

$$
Q_{+}:=\sqrt{\frac{Q+1}{2}}=\sqrt{\frac{1}{2} \cdot \frac{2}{1-z^{2} \exp \left(-\frac{1}{2} H \beta\right)}}=\frac{1}{\sqrt{1-z^{2} \exp \left(-\frac{1}{2} H \beta\right)}}
$$

and

$$
\begin{aligned}
Q_{-}:= & \iota \sqrt{\frac{Q-1}{2}}=\iota \sqrt{\frac{1}{2} \cdot \frac{2 \exp \left(-\frac{1}{2} H \beta\right)}{1-z^{2} \exp \left(-\frac{1}{2} H \beta\right)}} \\
& =z \iota \sqrt{\frac{\exp \left(-\frac{1}{2} H \beta\right)}{1-z^{2} \exp \left(-\frac{1}{2} H \beta\right)}}=: z Q^{-} .
\end{aligned}
$$

Moreover, we have

$$
A(f)=A\left(Q_{+} f\right) \otimes 1+1 \otimes A^{+}\left(Q_{-} f\right) .
$$

So up to a unitary isomorphism:

$$
\begin{aligned}
& A^{+}(f) A(g)=\left(A^{+}\left(Q_{+} f\right) \otimes 1+1 \otimes A\left(Q_{-} f\right)\right) . \\
& \cdot\left(A\left(Q_{+} g\right) \otimes 1+1 \otimes A^{+}\left(Q_{-} g\right)\right) \\
&= A^{+}\left(Q_{+} f\right) A\left(Q_{+} g\right) \otimes 1+z\left(A^{+}\left(Q_{+} f\right) \otimes A^{+}\left(Q^{-} g\right)\right. \\
&\left.+A\left(Q_{+} g\right) \otimes A\left(Q^{-} f\right)\right)+z^{2} 1 \otimes A\left(Q^{-} f\right) A^{+}\left(Q^{-} g\right) .
\end{aligned}
$$

By the CCR, the last term of the right hand side of (2.11) is equal to

$$
z^{2} 1 \otimes\left[A^{+}\left(Q^{-} g\right) A\left(Q^{-} f\right)+\left\langle Q^{-} f, Q^{-} g\right\rangle_{\iota}\right]
$$

therefore the right hand side of (2.11) can be rewritten as 
(2.11a)

$$
\begin{aligned}
& \quad A^{+}\left(Q_{+} f\right) A\left(Q_{+} g\right) \otimes 1 \\
& \quad+z\left(A^{+}\left(Q_{+} f\right) \otimes A^{+}\left(Q^{-} g\right)+A\left(Q_{+} g\right) \otimes A\left(Q_{-} f\right)\right)+ \\
& \quad+z^{2}\left(1 \otimes A^{+}\left(Q^{-} g\right) A\left(Q^{-} f\right)+\left\langle Q^{-} f, Q^{-} g\right\rangle_{\iota}\right) \\
& =: A_{0}(f, g)+z\left(A_{-1}(f, g)+A_{1}(f, g)\right)+z^{2} A_{2}(f, g)+z^{2} A_{-2}(f, g) .
\end{aligned}
$$

Our starting point of this paper is, as in [1], the iterative solution of (1.5):

$$
U_{t}=\sum_{n=0}^{\infty}(-i)^{n} \int_{0}^{t} d t_{1} \cdots \int_{0}^{t_{n-1}} d t_{n} V\left(t_{1}\right) \cdots V\left(t_{n}\right)
$$

Now, we want to show what is the difference between the Fock and non-Fock cases.

In (2.11a), the term $A_{0}(f, g)$ is similar to that in the Fock case and the term $z^{2} A_{ \pm 2}(f, g)$ will, roughly speaking, not play any important role. In the term $z\left(A_{-1}(f, g)+A_{1}(f, g)\right)$, if there exists some $A\left(Q^{-} g\right)$ acting on $W\left(z \int_{S / z^{2}}^{T / z^{2}}\right.$ $\left.Q_{-} S_{u} f d u\right)$ or $W\left(z \int_{S^{\prime} / z^{2}}^{T^{\prime} / Q^{2}} Q_{-} S_{u} f^{\prime} d u\right)$, since $Q_{-}=z Q^{-}$and $Q^{-}$tends to $c$ 。 $\exp \left(-\frac{1}{4} \beta H\right)$ as $z \rightarrow 0$, we shall get so "many" $z$ that the term will go to zero. But the new situation is not the same as that in the Fock case: there it is not possible that all the operators are "used" to produce scalar products, but here is possible. For example, in the case of $n=2$, we can get the following term

$$
A\left(S_{t_{1}} Q+g_{0}\right) \otimes A\left(Q-S_{t_{1}} g_{1}\right) \cdot A^{+}\left(S_{t_{2}} Q+g_{0}\right) \otimes A^{+}\left(Q-S_{t_{2}} g_{1}\right) .
$$

From it one can get the following term.

$$
\left\langle Q+g_{0}, S_{t_{2}-t_{2}} Q+g_{0}\right\rangle \cdot\left\langle\mathrm{Q}-g_{1}, Q-S_{t_{2}-t_{2}} g_{1}\right\rangle_{\iota}
$$

So in the low density limit, the following term will be obtained

$$
\begin{gathered}
\lim _{z \rightarrow 0} \int_{0}^{t / z^{2}} d t_{1} \int_{0}^{t_{1}} d t_{2}\left\langle Q+g_{0}, S_{t_{2}-t_{1}} Q+g_{0}\right\rangle \cdot\left\langle Q-g_{1}, Q-S_{t_{2}-t_{1}} g_{1}\right\rangle_{\iota} \\
\lim _{z \rightarrow 0} \int_{0}^{t / z^{2}} d t_{1} \int_{0}^{t_{1}} d t_{2} z^{2}\left\langle Q+g_{0}, S_{t_{2}-t_{1}} Q+g_{0}\right\rangle \cdot\left\langle Q^{-} g_{1}, Q^{-} S_{t_{2}-t_{1}} g_{1}\right\rangle_{\iota} \\
\lim _{z \rightarrow 0} \int_{0}^{t} d t_{1} \int_{-t_{1} / z^{2}}^{0} d t_{2}\left\langle Q+g_{0}, S_{t_{2}} Q+g_{0}\right\rangle \cdot\left\langle Q^{-} g_{1}, Q^{-} S_{t_{2}} g_{1}\right\rangle_{\iota} \\
=\int_{0}^{t} d t_{1} \int_{-\infty}^{0} d u\left\langle g_{0}, S_{u} g_{0}\right\rangle \cdot \overline{\left\langle e^{-\frac{1}{4} \beta H} g_{1}, S_{u} e^{-\frac{1}{4} \beta H} g_{1}\right\rangle}
\end{gathered}
$$

In the case of $n \geq 2$, one gets similar terms. 


\section{§ 3. The collective terms and negligible terms}

The section is devoted to distinguish the collective terms from the negligible terms. First of all by (1.20) and (1.7), for each $n \in \mathbf{N}, \varepsilon \in\{0,1\}^{n}$ up to a unitary isomorphism,

$$
\begin{gathered}
=\left(A^{+}\left(S_{t_{1}} Q_{+} g_{\varepsilon(1)}\right) A\left(S_{t 1} Q_{+} g_{1-\varepsilon(1)}\right) \otimes 1+z\left(A^{+}\left(S_{t_{1}} Q_{+} g_{\varepsilon(1)}\right) \otimes A^{+}\left(Q^{-} S_{t 1} g_{1-\varepsilon(1)}\right)\right.\right. \\
\left.+A\left(S_{t 1} Q_{+} g_{1-\varepsilon(1)}\right) \otimes A\left(Q^{-} S_{t 1} g_{\varepsilon(1)}\right)\right) \\
\left.+z^{2} 1 \otimes\left(A^{+}\left(S_{t_{1}} Q^{-} g_{1-\varepsilon(1)}\right) A\left(Q^{-} S_{t_{1}} g_{\varepsilon(1)}\right)+\left\langle Q^{-} g_{\varepsilon(1)}, Q^{-} g_{1-\varepsilon(1)}\right\rangle_{\iota}\right)\right) \cdots \cdots \\
\left(A^{+}\left(S_{t n} Q+g_{\varepsilon(n)}\right) A\left(S_{t n} Q_{+} g_{1-\varepsilon(n)}\right) \otimes 1+z\left(A^{+}\left(S_{t_{n}} Q_{+} g_{\varepsilon(n)}\right) \otimes A^{+}\left(Q^{-} S_{t n} g_{1-\varepsilon(n)}\right)\right.\right. \\
\left.\quad+A\left(S_{t n} Q_{+} g_{1-\varepsilon(n)}\right) \otimes A\left(Q^{-} S_{t n} g_{\varepsilon(n)}\right)\right) \\
\left.+z^{2} 1 \otimes\left(A^{+}\left(Q^{-} S_{t n} g_{1-\varepsilon(n)}\right) A\left(Q^{-} S_{t n} g_{\varepsilon(n)}\right)+\left\langle Q^{-} g_{\varepsilon(n)}, Q^{-} g_{1-\varepsilon(n)}\right\rangle_{\iota}\right)\right) .
\end{gathered}
$$

Expanding the right hand side of (3.1) and using the notations in the right hand side of $(1.20 \mathrm{a})$, one can write (3.1) to

$$
\sum_{\sigma \in\{0, \pm 1, \pm 2\}^{n}} A_{\sigma(1)}\left(S_{t 1} g_{\varepsilon(1)}, S_{t 1} g_{1-\varepsilon(1)}\right) \cdots A_{\sigma(n)}\left(S_{t n} g_{\varepsilon(1)}, S_{t n} g_{1-\varepsilon(n)}\right) z^{\Sigma_{k=1}^{n}|\sigma(k)|} .
$$

In order to write (3.2) to a more clear form, let us label $A_{1}$ by $1 \leq i_{1}<\cdots<i_{k}$ $\leq n ; A_{-1}$ by $1 \leq i_{1}^{\prime}<\cdots<i_{k^{\prime}}^{\prime} \leq n ; A_{2}$ by $1 \leq i_{1}^{\prime \prime}<\cdots<i_{k^{\prime \prime}}^{\prime \prime} \leq n$ and $A_{-2}$ by $1 \leq i_{1}^{\prime \prime \prime}<\cdots<i_{k^{\prime \prime \prime}}^{\prime \prime \prime} \leq n$, then the terms $A_{0}$ are naturally labeled by the indices set $\left.\{1, \ldots, n\} \backslash\left(i_{h}\right\}_{h=1}^{k} \cup\left(i_{h}^{\prime}\right\}_{h=1}^{k^{\prime}} \cup\left(i_{h}^{\prime \prime}\right\}_{h=1}^{k^{\prime \prime}} \cup\left(i_{h}^{\prime \prime \prime}\right\}_{h=1}^{k^{\prime \prime \prime}}\right)$. Thus (3.2) is equal to the following expression

$$
\begin{aligned}
& \sum_{k=0}^{n} \sum_{1 \leq i_{1}<\cdots<i_{k} \leq n} \sum_{k^{\prime}=0}^{n-k} \sum_{1 \leq i_{1}<\cdots<i_{k^{\prime}} \leq n} \sum_{k^{\prime \prime}=0}^{n-k-k^{\prime}} \sum_{1 \leq i^{\prime \prime}<\cdots<i_{k^{\prime \prime}}^{\prime \prime} \leq n} \\
& \left\{i_{h}\right\}_{h=1}^{k} \cap\left\{i_{h}^{\prime}\right\}_{h=1}^{k^{\prime}=\varnothing} \quad\left\{i_{h}^{\prime}\right\}_{h=1}^{k^{\prime \prime}} \cap\left(\left\{i_{h}\right\}_{h=1}^{k} \cup\left(i^{\prime}\right\}_{h=1}^{k^{\prime}}\right)=\varnothing \\
& \sum_{k^{\prime \prime \prime}=0}^{n-k-k^{\prime}-k^{\prime \prime}} \sum_{1 \leq i_{1}^{\prime \prime \prime}<\cdots<i_{k^{\prime \prime \prime}}^{\prime \prime \prime} \leq n} \\
& \left\{i i_{h}^{\prime \prime \prime}\right\}_{h=1}^{k^{\prime \prime \prime}} \cap\left(\{i h\}_{h=1}^{k} \cup\left\{i_{h}^{\prime}\right\}_{h=1}^{k^{\prime}} \cup\left\{i_{h}^{\prime \prime}\right\}_{h=1}^{\prime \prime}\right)=\varnothing \\
& z^{k+k^{\prime}+2\left(k^{\prime \prime}+k^{\prime \prime \prime}\right)} A_{0}\left(S_{t_{1}} g_{\varepsilon(1)}, S_{t_{1}} g_{1-\varepsilon(1)}\right) \cdots A_{1}\left(S_{t_{1}} g_{\varepsilon\left(i_{1}\right)}, S_{t_{1}} g_{1-\varepsilon\left(i_{1}\right)}\right) \cdots \\
& A_{-1}\left(S_{t_{t_{1}}} g_{\varepsilon\left(i^{\prime}\right)}, S_{t_{i_{1}}} g_{1-\varepsilon\left(i_{1}^{\prime}\right)}\right) \cdots A_{2}\left(S_{t_{i^{\prime}}} g_{\varepsilon\left(i_{1}^{\prime}\right)}, S_{t_{i^{\prime}}} g_{1-\varepsilon\left(i_{1}^{\prime}\right)}\right) \cdots
\end{aligned}
$$




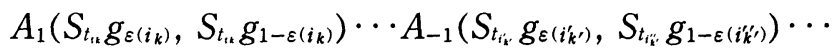

$$
\begin{aligned}
& A_{2}\left(S_{t_{t^{\prime \prime}}} g_{\varepsilon\left(i^{\prime \prime \prime}\right)}, S_{t_{t^{\prime \prime},}} g_{1-\varepsilon\left(i^{\prime \prime \prime} k^{\prime \prime}\right)}\right) \cdots A_{0}\left(S_{t_{n}} g_{\varepsilon(n)} S_{t_{n}} g_{1-\varepsilon(n)}\right) \\
& \prod_{h=1}^{k^{\prime \prime \prime}}\left\langle Q^{-} g_{\varepsilon\left(i_{h}^{\prime \prime \prime}\right)}, Q^{-} g_{1-\varepsilon\left(i_{h}^{\prime \prime \prime}\right)}\right\rangle_{c} \\
& =\sum_{k=0}^{n} \sum_{1 \leq i_{1}<\cdots<i_{k} \leq n} \sum_{k^{\prime}=0}^{n-k} \sum_{1 \leq i_{1}^{\prime}<\cdots<i^{\prime} k^{\prime} \leq n} \sum_{k^{\prime \prime}=0}^{n-k-k^{\prime}} \sum_{1 \leq i_{1}^{\prime \prime}<\cdots<i_{k^{\prime \prime}}^{\prime \prime} \leq n} \\
& \left\{i h_{h=1}^{k} \cap\left\{i_{h}^{\prime}\right\}_{h=1}^{k^{\prime}=\emptyset} \quad\left\{i^{\prime} h_{h=1}^{\prime}\right\}^{\prime \prime} \cap\left(\{i h\}_{h=1}^{k} \bigcup\left\langle i_{h}^{\prime}\right\}_{h=1}^{k^{\prime}}\right)=\emptyset\right. \\
& \sum_{k^{\prime \prime \prime}=0}^{n-k-k^{\prime}-k^{\prime \prime}} \sum_{1 \leq i 1^{\prime \prime}<\cdots<i^{\prime \prime \prime \prime \prime} \leq n}
\end{aligned}
$$

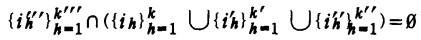

$$
\begin{aligned}
& z^{k+k^{\prime}+2\left(k^{\prime \prime}+k^{\prime \prime \prime}\right)} A^{+}\left(S_{t_{1}} Q+g_{\varepsilon(1)}\right) A\left(S_{t_{1}} Q+g_{1-\varepsilon(1)}\right) \cdots A\left(S_{t_{11}} Q+g_{1-\varepsilon\left(i_{1}\right)}\right) \\
& \cdots A^{+}\left(S_{t_{t_{i}}} Q+g_{\left.\varepsilon\left(t^{\prime}\right)\right)}\right) \cdots A\left(S_{t_{t}} Q+g_{1-\varepsilon\left(i_{k}\right)}\right) \\
& \cdots A^{+}\left(S_{t_{i,}} Q+g_{\varepsilon\left(i^{\prime} k^{\prime}\right)}\right) \cdots A^{+}\left(S_{t_{n}} Q+g_{\varepsilon(n)}\right) \mathrm{A}\left(S_{t_{n}} Q+g_{1-\varepsilon(n)}\right) \\
& \otimes A\left(Q-S_{t_{i}} g_{\varepsilon\left(i_{1}\right)}\right) \cdots A^{+}\left(Q^{-} S_{t_{i_{1}}} g_{\left.1-\varepsilon\left(i^{\prime}\right)\right)}\right) \cdots \\
& A\left(Q^{-} S_{t_{i^{\prime}}} g_{1-\varepsilon\left(i^{\prime \prime}\right)}\right) A^{+}\left(Q^{-} S_{t_{i_{i}}} g_{\varepsilon\left(i^{\prime \prime}\right)}\right) \cdots \\
& A\left(Q-S_{t_{t}} g_{\varepsilon\left(i_{k}\right)}\right) \cdots A+\left(Q-S_{t_{i^{\prime}}} g_{1-\varepsilon\left(i^{\prime} k^{\prime}\right)}\right) \cdots
\end{aligned}
$$

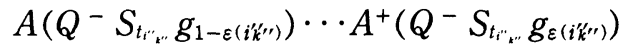

$$
\begin{aligned}
& \prod_{h=1}^{k^{\prime \prime \prime}}\left\langle Q^{-} g_{\varepsilon\left(i_{h}^{\prime \prime \prime}\right)}, Q^{-} g_{1-\varepsilon\left(i_{h}^{\prime \prime \prime}\right)}\right\rangle \text { 。 } \\
& =: \mathrm{I}_{n}^{\varepsilon}+\mathrm{II}_{n}^{\varepsilon}
\end{aligned}
$$

where, $I_{n}^{\varepsilon}$ denotes those terms satisfying $k^{\prime \prime}=0$ and $\mathrm{II}_{n}^{\varepsilon}$ denotes those terms satisfying $k^{\prime \prime} \geq 1$.

Now, we write $\mathbf{I}_{n}^{\varepsilon}$ and $\mathrm{II}_{n}^{\varepsilon}$ in normally ordered form. Then, one has

Lemma (3.1). For each $n \in \boldsymbol{N}, \varepsilon \in\{0,1\}^{n}$,

$$
\begin{aligned}
& \mathrm{I}_{n}^{\varepsilon}=\sum_{k=0}^{n} \sum_{1 \leq i_{1}<\cdots<i_{k} \leq n} \sum_{k^{\prime}=0}^{n-k} \sum_{1 \leq i_{1}^{\prime}<\cdots<i_{k^{\prime}} \leq n} \sum_{k^{\prime \prime \prime}=0}^{n-k-k^{\prime}} \sum_{1 \leq i^{\prime \prime}<\cdots<i^{\prime \prime \prime \prime} \leq n}
\end{aligned}
$$

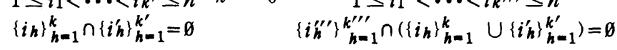

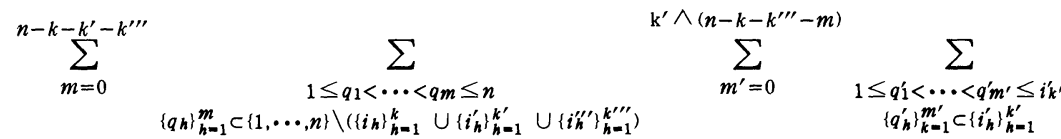

$$
\begin{aligned}
& \sum_{\left(p_{1}, \cdots, p_{m}, p_{1}^{\prime}, \cdots, p_{m^{\prime}}\right)} z^{k+k^{\prime}}
\end{aligned}
$$




$$
\begin{aligned}
& \prod_{h=1}^{m}\left\langle S_{t_{p}} Q+g_{1-\varepsilon\left(p_{h}\right)}, S_{t_{\varphi_{1}}} Q_{+} g_{\varepsilon\left(q_{h}\right)}\right\rangle \cdot \prod_{h=1}^{m^{\prime}}\left\langle S_{t_{p_{i}}} Q_{+} g_{1-\varepsilon\left(p_{h}^{\prime}\right)}, S_{t_{\gamma_{i}}} Q+g_{\varepsilon\left(q_{h}^{\prime}\right)}\right\rangle \\
& \prod_{1}^{+}\left(S_{t_{\alpha}} Q+g_{\varepsilon(\alpha)}\right) \\
& \alpha \in\left\{1, \cdots, n \backslash \backslash\left\{i_{h}\right\}_{h=1}^{k} \cup\left\{i_{h}^{\prime}\right\}_{h=1}^{k^{\prime}} \cup\left\{i_{h}^{\prime \prime} h_{h=1}^{k^{\prime \prime}} \cup\left\{q_{h}\right\}_{h=1}^{m}\right\}\right. \\
& \prod_{\alpha \in\left\{i_{h}^{\prime}\right\}_{h=1}^{k^{\prime}} \backslash\left\{q_{h}^{\prime}\right\}_{h=1}^{m^{\prime}}} A^{+}\left(S_{t_{\alpha}} Q+g_{\varepsilon(\alpha)}\right) \\
& \Pi \quad A\left(S_{t_{\alpha}} Q+g_{1-\varepsilon(\alpha)}\right) \\
& \alpha \in\left\{1, \cdots, n \nmid \backslash\left\{i_{h}^{\prime}\right\}_{h=1}^{\prime} \cup\left\{i_{h}^{\prime \prime \prime}\right\}_{h=1}^{k \prime \prime \prime} \cup\left\{p_{h}\right\}_{h=1}^{m} \cup\left\{p_{h}^{\prime}\right\}_{h=1}^{m^{\prime}}\right\}
\end{aligned}
$$

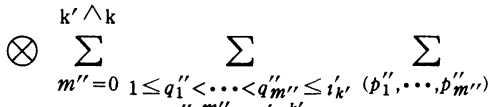

$$
\begin{aligned}
& \left\{q_{h}^{\prime \prime}\right\}_{h=1}^{m^{\prime \prime}} \subset\left\{i_{h}^{\prime} h_{h=1}^{k^{\prime}}\right. \\
& \prod_{h=1}^{m}\left\langle Q^{-} S_{t_{p^{\prime}}} g_{\varepsilon\left(p_{h}^{\prime \prime}\right)}, Q^{-} S_{t_{o^{\prime}}} g_{1-\varepsilon\left(q_{h}^{\prime \prime}\right)}\right\rangle_{c}
\end{aligned}
$$

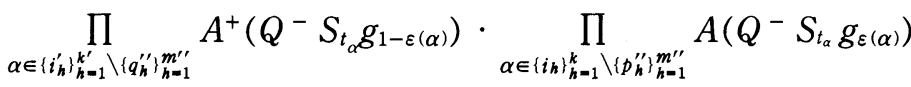

$$
\begin{aligned}
& z^{2 k^{\prime \prime \prime}} \prod_{h=1}^{k^{\prime \prime \prime}}\left\langle Q^{-} g_{1-\varepsilon\left(i h^{\prime}\right)}, Q^{-} g_{\varepsilon\left(i h^{\prime \prime}\right)}\right\rangle_{\iota}
\end{aligned}
$$

where, $\sum_{\left(p_{1}, \cdots, p_{m}, p_{1}^{\prime}, \cdots, p^{\prime} m^{\prime}\right)}$ means the sum for all $1 \leq p_{1}, \cdots, p_{m}, p_{1}^{\prime}, \cdots p_{m^{\prime}}^{\prime} \leq n$ which satisfy

(i) $\left\{p_{h}\right\}_{h=1}^{m} \bigcup\left\{p_{h}^{\prime}\right\}_{h=1}^{m^{\prime}} \subset\{1, \ldots, n\} \backslash\left(\left\{i_{h}^{\prime}\right\}_{h=1}^{k^{\prime}} \cap\left\{i_{h=1}^{\prime \prime \prime}\right\}_{h=1}^{k^{\prime \prime \prime}}\right)$;

(ii) $\left|\left\{p_{h}\right\}_{h=1}^{m} \cup\left\{p_{h}^{\prime}\right\}_{h=1}^{m^{\prime}}\right|=m+m^{\prime}$;

(iii) $p_{h}<q_{h}$ for all $h=1, \ldots, m$ and $p_{h}^{\prime}<q_{h}^{\prime}$ for all $h=1, \ldots, m^{\prime}$;

and $\sum_{\left(p_{1}^{\prime \prime}, \cdots, P_{m}^{\prime \prime}\right)}$ means the sum for all $1 \leq p_{1}^{\prime \prime}, \cdots, p_{m}^{\prime \prime} \leq n$ satisfying

(i) $\left\{p_{h}^{\prime \prime}\right\}_{h=1}^{m^{\prime \prime}} \subset\left\{i_{h}\right\}_{h=1}^{k}$;

(ii) $\left|\left\{p_{h}^{\prime \prime}\right\}_{h=1}^{m^{\prime \prime}}\right|=m^{\prime \prime}$;

(iii) $p_{h}^{\prime \prime}<q_{h}^{\prime \prime}$ for all $h=1, \ldots, m^{\prime \prime}$.

Proof. Notice that the indices $\left\{i_{h}^{\prime \prime \prime}\right\}_{h=1}^{k^{\prime \prime \prime}}$ label only scalar product terms which can be thought as "normally ordered", therefore, we can assume, without loss of generality, that $k^{\prime \prime \prime}=0$.

By (3.3) and the definition of $\mathrm{I}_{n}^{\varepsilon}\left(k^{\prime \prime}=0\right)$, one has

$$
\begin{gathered}
\mathrm{I}_{n}^{\varepsilon}=\sum_{k=0}^{n} \sum_{1 \leq i_{1}<\cdots<i_{k} \leq n} \sum_{k^{\prime}=0}^{n-k} \sum_{\substack{1 \leq i_{1}<\cdots<i^{\prime} k^{\prime} \leq n \\
\left\{i h _ { h = 1 } ^ { k } \cap \left\{i_{h}^{\prime} k_{h=1}^{\prime}=\emptyset\right.\right.}} z^{k+k^{\prime}} \\
\sum_{m=0}^{n-k-k^{\prime}} C_{n}(1, m)\left(A^{+\cdots}\right)_{\left(n-k-k^{\prime}-m\right)} . \\
\sum_{m^{\prime}=0}^{k^{\prime}} C_{n}\left(2, m^{\prime}\right)\left(A^{+\cdots}\right)_{\left(k^{\prime}-m^{\prime}\right)} \cdot(A \cdots)_{\left(n-k-k^{\prime}+k-m-m^{\prime}\right)}
\end{gathered}
$$




$$
\otimes \sum_{m^{\prime \prime \prime}} C_{n}\left(3, m^{\prime \prime}\right)\left(A^{+\cdots}\right)_{\left(k^{\prime}-m^{\prime \prime}\right)} \cdot(A \cdots)_{\left(k-m^{\prime \prime}\right)}
$$

where, $C_{n}(1, m), C_{n}\left(2, m^{\prime}\right)$ and $C_{n}\left(3, m^{\prime \prime}\right)$ are the products of some scalar products; $\left(A^{+} \cdots\right)_{\left(n-k-k^{\prime}-m\right)}$ is the product of the creators which is a $n-k-k^{\prime}-$ $m$ 's elements subset of

$$
\left\{A^{+}\left(S_{t_{a}} Q^{+} g_{\varepsilon(\alpha)}\right) ; \alpha \in\{1, \cdots, n\} \backslash\left(\left\{i_{h}\right\}_{h=1}^{k} \bigcup\left\{i_{h}^{\prime}\right\}_{h=1}^{k^{\prime}}\right)\right\}
$$

whose complement is used to produce the scalar products; $\left(A^{+} \cdots\right)_{\left(k^{\prime}-m^{\prime}\right)}$ is the product of the creators which is a $k^{\prime}-m$ 's elements subset of

$$
\left\{A^{+}\left(S_{t_{i}} Q^{+} g_{\varepsilon\left(i_{h}^{\prime}\right)}\right)\right\}_{h=1}^{k^{\prime}}
$$

whose complement is used to produce the scalar products. So, $m \leq n-k-k^{\prime}$ and $m^{\prime} \leq k^{\prime}$. For each fixed $m$, one can use the set $\left\{q_{h}\right\}_{h=1}^{m} \subset\{1, \ldots, n\} \backslash\left(\left\{i_{h}\right\}_{h=1}^{k}\right.$ $\bigcup\left\{i_{h}^{\prime}\right\}_{h=1}^{k^{\prime}}$ ) to label the set of creators

$$
\left\{A^{+}\left(S_{t_{\alpha}} Q^{+} g_{\varepsilon(\alpha)}\right) ; \alpha \in\{1, \ldots, n\} \backslash\left(\left\{i_{h}\right\}_{h=1}^{k} \cup\left\{i_{h}^{\prime}\right\}_{h=1}^{k^{\prime}}\right)\right\} \backslash\left(A^{+} \cdots\right)_{\left(n-k-k^{\prime}-m\right)}
$$

Without loss of generality, we can suppose that

$$
1 \leq q_{1}<\cdots<q_{m} \leq n .
$$

For each fixed $m^{\prime}$, one can use the set $\left\{q_{h}^{\prime}\right\}_{h=1}^{m^{\prime}} \subset\left\{i_{h}^{\prime}\right\}_{h=1}^{k^{\prime}}$ to label the set of creators

$$
\left\{A^{+}\left(S_{t_{\mathrm{B}}} Q^{+} g_{\varepsilon\left(i_{h}^{\prime}\right)}\right)\right\}_{h=1}^{k^{\prime}} \backslash\left(A^{+\cdots}\right)_{\left(k^{\prime}-m^{\prime}\right)}
$$

Without loss of generality, we can suppose that

$$
1 \leq q_{1}^{\prime}<\cdots<q_{m^{\prime}}^{\prime} \leq n .
$$

For each fixed $m, m^{\prime}, 1 \leq q_{1}<\cdots<q_{m} \leq n, 1 \leq q_{1}^{\prime}<\cdots q_{m^{\prime}}^{\prime}<n$, there is a $m$ $+m^{\prime \prime}$ s elements subset of annihilators

$$
\left.\left\{A\left(S_{t_{\alpha}} Q^{+} g_{\varepsilon(\alpha)}\right) ; \alpha \in\{1, \ldots, n\} \backslash\left\{i_{h}\right\}_{h=1}^{k} \cup\left\{i_{h}^{\prime}\right\}_{h=1}^{k^{\prime}}\right)\right\}
$$

which is used to produce scalar products with

$$
\left.\left\{A^{+}\left(S_{t_{\alpha}} Q^{+} g_{\varepsilon(\alpha)}\right) ; \alpha \in\{1, \ldots, n\} \backslash\left\{i_{h}\right\}_{h=1}^{k} \bigcup\left\{i_{h}^{\prime}\right\}_{h=1}^{k^{\prime}}\right)\right\} \backslash\left\{A^{+}\left(S_{t_{i_{\alpha}}} Q^{+} g_{\varepsilon\left(i_{h}^{\prime \prime}\right)}\right)\right\}_{h=1}^{k^{\prime}}
$$

If we label the subset by $\left\{p_{h}\right\}_{h=1}^{m}$ and $\left\{p_{h}^{\prime}\right\}_{h=1}^{m^{\prime}}$ respectively, then, $1 \leq p_{1}, \ldots, p_{m}$, $p_{1}^{\prime}, \ldots, p_{m^{\prime}} \leq n ;\left\{p_{h}\right\}_{h=1}^{m} \cup\left\{p_{h}^{\prime}\right\}_{h=1}^{m} \subset\{1, \ldots, n\} \backslash\left\{i_{h}^{\prime}\right\}_{h=1}^{k^{\prime}}$ and $\left|\left\{p_{h}\right\}_{h=1}^{m} \cup\left\{p_{h}^{\prime}\right\}_{h=1}^{m^{\prime}}\right|=$ $m+m^{\prime}$. Moreover, $m+m^{\prime} \leq n-k^{\prime}$, i.e. $m^{\prime} \leq n-k^{\prime}-m$. So, we obtain the following 


$$
\begin{aligned}
& \mathrm{I}_{n}^{\varepsilon}=\sum_{k=0}^{n} \sum_{1 \leq i_{1}<\cdots<i_{k} \leq n} \sum_{k^{\prime}=0}^{n-k} \sum_{1 \leq i_{1}^{\prime}<\cdots<i_{k^{\prime}} \leq n} \\
& \left\{i_{h}\right\}_{h=1}^{k} \cap\left\{i_{h}^{\prime}\right\}_{h=1}^{k^{\prime}}=\varnothing \\
& \sum_{m=0}^{n-k-k^{\prime}} \sum_{\substack{\left.1 \leq q_{1}<\cdots<q_{m} \leq n \\
\left\{q_{h}\right\}_{h=1}^{m} \subset\{1, \cdots, n\} \backslash\left(i_{h}\right\}_{h=1}^{k} \cup\left\{i_{h}^{\prime}\right\}^{k^{\prime}}\right\}_{h=1}^{\prime}}} \sum_{m^{\prime}=0}^{k^{\prime} \wedge\left(n-k^{\prime}-m\right)} \sum_{\substack{1 \leq q_{1}^{\prime}<\cdots<q_{m^{\prime}}^{\prime} \leq i_{k^{\prime}}^{\prime} \\
\left\{q_{h^{\prime}}^{\prime}\right\}_{h=1}^{m^{\prime}} \subset\left\{i_{h}^{\prime}\right\}_{h=1}^{k^{\prime}}}} \\
& \sum_{\left(p_{1}, \cdots, p_{m}, p_{1}^{\prime}, \cdots, p_{m^{\prime}}^{\prime}\right)} z^{k+k^{\prime}}
\end{aligned}
$$

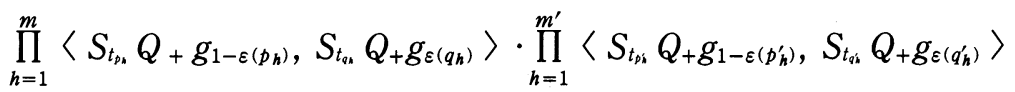

$$
\begin{aligned}
& \prod_{\left.\alpha \in\{1, \cdots, n\} \backslash\left\{i_{h}\right)_{h=1}^{k} \cup\left\{i_{h}^{\prime}\right\}_{h-1}^{\prime} \cup\left\{q_{h}\right\}_{h=1}^{m}\right)} A^{+}\left(S_{t_{\alpha}} Q+g_{\varepsilon(\alpha)}\right) \\
& \prod_{\alpha \in\left\{i_{h}^{\prime}\right\}_{h=1}^{k^{\prime}} \backslash\left\{q_{h}^{\prime}\right\}_{h=1}^{m^{\prime}}} A^{+}\left(S_{t_{\alpha}} Q+g_{\varepsilon(\alpha)}\right) \\
& \Pi \quad A\left(S_{t_{\alpha}} Q+g_{1-\varepsilon(\alpha)}\right) \\
& \alpha \in\{1, \cdots, n\} \backslash\left(\left\{i_{h}^{\prime}\right)_{h=1}^{k^{\prime}} \cup\left(p_{h}\right\}_{h=1}^{m} \cup\left\{p_{h}^{\prime}\right\}_{h=1}^{m^{\prime}}\right) \\
& \otimes \sum_{m^{\prime \prime}} C_{n}\left(3, m^{\prime \prime}\right)\left(A^{+\cdots}\right)_{\left(k^{\prime}-m^{\prime \prime}\right)} \cdot(A \cdots)_{\left(k-m^{\prime \prime}\right)} \text {. }
\end{aligned}
$$

Similarly, we can deal with

$$
\sum_{m^{\prime \prime}} C_{n}\left(3, m^{\prime \prime}\right)\left(A^{+} \cdots\right)_{\left(k^{\prime}-m^{\prime \prime}\right)} \cdot(A \cdots)_{\left(k-m^{\prime \prime}\right)}
$$

and get that (3.12) is equal to

$$
\begin{aligned}
& \sum_{m^{\prime \prime}=0}^{k^{\prime} \wedge k} \sum_{1 \leq q_{1}^{\prime \prime}<\cdots<q_{m^{\prime \prime}}^{\prime \prime} \leq i_{k^{\prime}}} \sum_{p_{1}^{\prime \prime}, \cdots, p_{m^{\prime \prime}}^{\prime \prime}} \\
& \left\{q_{h}^{\prime \prime}\right\}_{h=1}^{m^{\prime \prime}}<\left\{i_{h}^{\prime}\right\}_{h=1}^{k^{\prime}} \\
& \prod_{h=1}^{m}\left\langle Q^{-} S_{t p_{h}^{\prime \prime}} g_{\varepsilon\left(p_{h}^{\prime \prime}\right)}, Q^{-} S_{t_{q^{\prime}}} g_{\varepsilon\left(q_{h}^{\prime \prime}\right)}\right\rangle_{\iota} \\
& \prod_{\alpha \in\left\{i_{h}^{\prime}\right\}_{h=1}^{k^{\prime}} \backslash\left\{q_{h}^{\prime \prime}\right\}_{h=1}^{m^{\prime \prime}}} A^{+}\left(Q^{-} S_{t_{\alpha}} g_{1-\varepsilon(\alpha)}\right) \cdot \prod_{\alpha \in\left\{i_{h}\right\}_{h=1}^{k} \backslash\left\{p^{\prime \prime}\right\}_{h=1}^{m^{\prime \prime}}} A^{+}\left(Q^{+} S_{t_{\alpha}} g_{\varepsilon(\alpha)}\right) \cdot
\end{aligned}
$$

Combining together (3.12) and (3.13a), one finishes the proof.

As an appication of this Lemma, we can easily obtain the following

Corollary (3.2). For each $n \in \mathbf{N}, \varepsilon \in\{0,1\}^{n}, f, f^{\prime} \in K, T, S, T^{\prime}, S^{\prime} \in \mathbf{R}$,

$$
\left\langle W\left(z \int_{S / z^{2}}^{T / z^{2}} Q_{+} S_{u} f d u\right) \Phi_{F} \otimes W\left(z^{2} \int_{S / z^{2}}^{T / z^{2}} Q^{-} S_{u} f d u\right) \Phi_{F}^{\iota},\right.
$$




$$
\begin{aligned}
& \int_{0}^{t / z^{2}} d t_{1} \int_{0}^{t_{1}} d t_{2} \cdots \int_{0}^{t_{n-1}} d t_{n} \mathrm{I}_{n}^{\varepsilon} \\
& \left.\cdot W\left(z \int_{S^{\prime} / z^{2}}^{T^{\prime} / z^{2}} Q+S_{u} f^{\prime} d u\right) \Phi_{F} \otimes W\left(z^{2} \int_{S^{\prime} / z^{2}}^{T^{\prime} / z^{2}} Q^{-} S_{u} f^{\prime} d u\right) \Phi_{F}^{\iota}\right\rangle \\
& =\sum_{k=0}^{n} \sum_{1 \leq i_{1}<\cdots<i_{k} \leq n} \sum_{k^{\prime}=0}^{n-k} \sum_{1 \leq i_{1}^{\prime}<\cdots<i_{k^{\prime}} \leq n} \sum_{k^{\prime \prime \prime}=0}^{n-k-k^{\prime}} \sum_{1 \leq i_{1}^{\prime \prime \prime}<\cdots<i_{k^{\prime \prime \prime}}^{\prime \prime \prime} \leq n} \\
& \{i h\}_{h=1}^{k} \cap\left\{i_{h}^{\prime}\right\}_{h=1}^{k^{\prime}=\emptyset} \quad\left\{i h_{h}^{\prime \prime \prime}\right\}_{h=1}^{k \prime \prime} \cap\left(\{i h\}_{h=1}^{k} \cup\left(i_{h}^{\prime}\right\}_{h=1}^{k^{\prime}}\right)=\emptyset
\end{aligned}
$$

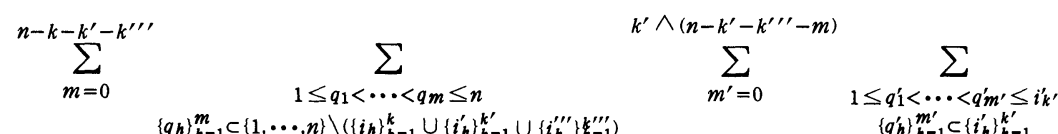

$$
\begin{aligned}
& \sum_{\left(p_{1}, \cdots, p m, p^{\prime}, \cdots, p^{\prime} m^{\prime}\right)} z^{k+k^{\prime}} \int_{0}^{t / z^{2}} d t_{1} \int_{0}^{t_{1}} d t_{2} \cdots \int_{0}^{t_{n-1}} d t_{n} \\
& \prod_{h=1}^{m}\left\langle S_{t_{p,}} Q+g_{1-\varepsilon\left(p_{h}\right)}, S_{t_{p,}} Q+g_{\varepsilon\left(q_{h}\right)}\right\rangle \cdot \prod_{h=1}^{m^{\prime}}\left\langle S_{t_{p i}} Q+g_{1-\varepsilon\left(p_{h}^{\prime}\right)}, S_{t_{p_{i}}} Q+g_{\varepsilon\left(q_{h}^{\prime}\right)}\right\rangle \\
& z^{n-k-k^{\prime}-m-k^{\prime \prime \prime}} \prod_{\alpha \in\left\{1, \cdots, n \nmid \backslash(i h\}_{h=1}^{k} \cup\left\{i_{h}\right\}_{h=1}^{k^{\prime}} \cup\left\{i h^{\prime \prime}\right\}_{h=1}^{k^{\prime \prime \prime} \cup} \cup\left\{q_{h}\right\}_{h=1}^{m}\right)} \int_{S / z^{2}}^{T / z^{2}} d u\left\langle S_{u} Q+f, S_{t_{\alpha}} Q+g_{\varepsilon(\alpha)}\right\rangle \\
& z^{k^{\prime}-m^{\prime}} \prod_{\alpha \in\left\{i h^{\prime}\right\}_{h=1}^{k^{\prime}} \backslash\left\langle q_{h}^{\prime}\right\}_{h=1}^{m^{\prime}}} \int_{S / z^{2}}^{T / z^{2}} d u\left\langle S_{u} Q+f, S_{t_{\alpha}} Q+g_{\varepsilon(\alpha)}\right\rangle \\
& z^{n-k^{\prime}-m-m^{\prime}-k^{\prime \prime \prime}} \\
& \prod_{\left.\alpha \in\{1, \cdots, n\} \backslash\left\langle i_{h}^{\prime} h_{h=1}^{k^{\prime}} \cup \cup i_{h}^{\prime \prime \prime}\right\}_{h=1}^{k^{\prime \prime \prime}} \cup\left\{p_{h}\right\}_{h=1}^{m} \cup\left\{p_{h}^{\prime}\right\rangle_{h=1}^{m^{\prime}}\right)} \int_{S / z^{2}}^{T / z^{2}} d u\left\langle S_{t_{\alpha}} Q_{+} g_{1-\varepsilon(\alpha)}, S_{u} Q_{+} f^{\prime}\right\rangle .
\end{aligned}
$$

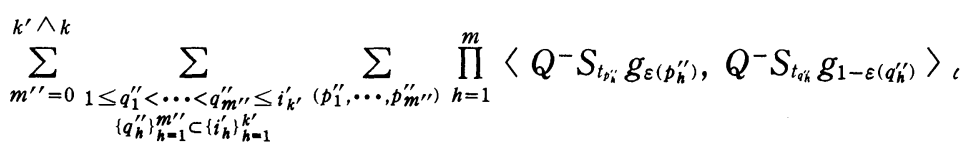

$$
\begin{aligned}
& z^{2\left(k^{\prime}-m^{\prime \prime}\right)} \prod_{\alpha \in\left\langle i_{h}^{\prime}\right\}_{h=1}^{k^{\prime}} \backslash\left\{q_{h}^{\prime \prime}\right\rangle_{h=1}^{m^{\prime \prime}}} \int_{S / z^{2}}^{T / z^{2}} d u\left\langle Q^{-} S_{u} f, Q^{-} S_{t_{\alpha}} g_{1-\varepsilon(\alpha)}\right\rangle_{i} \\
& \cdot z^{2\left(k^{\prime}-m^{\prime \prime}\right)} \prod_{\alpha \in\left\{i_{h}\right\}_{h=1}^{k} \backslash\left\langle p_{h}^{\prime \prime}\right\}_{h=1}^{m^{\prime \prime}}} \int_{S^{\prime} / z^{2}}^{T^{\prime} / z^{2}} d u\left\langle Q^{-} S_{t_{\alpha}} g_{\varepsilon(\alpha)}, Q^{-} S_{u} f\right\rangle_{\iota} \\
& z^{2 k^{\prime \prime}} \prod_{h=1}^{k^{\prime \prime \prime}}\left\langle Q^{-} g_{\varepsilon\left(i h^{\prime \prime}\right)}, Q^{-} g_{1-\varepsilon\left(i h^{\prime \prime}\right)}\right\rangle_{c} \\
& \left\langle W\left(z \int_{S / z^{2}}^{T / z^{2}} Q_{+} S_{u} f d u\right) \Phi_{F} \otimes W\left(z^{2} \int_{S / z^{2}}^{T / z^{2}} Q^{-} S_{u} f d u\right) \Phi_{F}^{\iota},\right. \\
& \left.W\left(z \int_{S^{\prime} / z^{2}}^{T^{\prime} / z^{2}} Q_{+} S_{u} f^{\prime} d u\right) \Phi_{F} \otimes W\left(z^{2} \int_{S^{\prime} / z^{2}}^{T^{\prime} / z^{2}} Q^{-} S_{u} f^{\prime} d u\right) \Phi_{F}^{\prime}\right\rangle
\end{aligned}
$$


Corollary (3.3). For each $n \in \mathbf{N}, \varepsilon \in\{0,1\}^{n}, f, f^{\prime} \in K, T, S, T^{\prime}, S^{\prime} \in \mathbf{R}$,

$$
\begin{aligned}
& \mid<W\left(z \int_{S / z^{2}}^{T / z^{2}} Q_{+} S_{u} f d u\right) \Phi_{F} \otimes W\left(z^{2} \int_{S / z^{2}}^{T / z^{2}} Q^{-} S_{u} f d u\right) \Phi_{F}^{\iota}, \\
& \int_{0}^{t / z^{2}} d t_{1} \int_{0}^{t_{1}} d t_{2} \cdots \int_{0}^{t_{n-1}} d t_{n} \mathrm{I}_{n}^{\varepsilon} \\
& \left.\cdot W\left(z \int_{S^{\prime} / z^{2}}^{T^{\prime} / z^{2}} Q_{+} S_{u} f^{\prime} d u\right) \Phi_{F} \otimes W\left(z^{2} \int_{S^{\prime} / z^{2}}^{T^{\prime} / z^{2}} Q^{-} S_{u} f^{\prime} d u\right) \Phi_{F}^{\iota}\right\rangle \mid \leq \\
& \leq \sum_{k=0}^{n} \sum_{1 \leq i_{1}<\cdots<i_{k} \leq n} \sum_{k^{\prime}=0}^{n-k} \sum_{1 \leq i_{1}^{\prime}<\cdots<i_{k^{\prime}} \leq n} \sum_{k^{\prime \prime \prime}=0}^{n-k-k^{\prime}} \sum_{1 s_{1}^{\prime \prime \prime}<\cdots<i_{k^{\prime \prime \prime \prime}}^{\prime \prime} \leq n} \\
& \left\{i h_{h=1}^{k} \cap\left\{i_{h}^{\prime}\right\}_{h=1}^{k^{\prime}=\emptyset} \quad\left\{i k^{\prime}\right\}_{h=1}^{k^{\prime \prime \prime}} \cap\left\{i_{h}\right\}_{h=n}^{k}\right\} \cup\left\{i_{h}^{\prime} h_{h=1}^{k^{\prime}}\right\}=\varnothing \\
& \sum_{m=0}^{n-k-k^{\prime}-k^{\prime \prime \prime}} \quad \sum_{1 \leq q_{1}<\cdots<q_{m} \leq n} \quad \sum_{m^{\prime}=0}^{\left.k^{\prime} \wedge n-k-k^{\prime}-k^{\prime \prime \prime}-m\right)}
\end{aligned}
$$

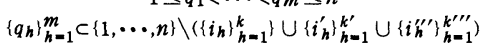

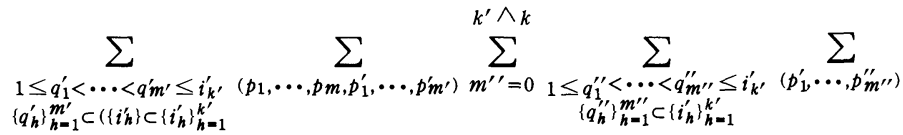

$$
\begin{aligned}
& z^{2\left(n+k+k^{\prime}-m-m^{\prime}-2 m^{\prime \prime}\right)} \cdot C_{1}^{2\left(n-m-m^{\prime}-m^{\prime \prime}\right)} \int_{0}^{t / z^{2}} d t_{1} \int_{0}^{t_{1}} d t_{2} \cdots \int_{0}^{t n-1} d t_{n} \\
& \prod_{h=1}^{m}\left|\left\langle S_{t_{\phi^{\prime}}} Q+g_{1-\varepsilon\left(p_{h}\right)}, S_{t q_{h}} Q+g_{\varepsilon\left(q_{h}\right)}\right\rangle\right| \cdot \prod_{h=1}^{\bar{m}}\left|\left\langle S_{t_{a,}} Q_{+} g_{1-\varepsilon\left(\alpha_{h}\right)}, S_{t_{t_{k}}} Q_{+} g_{\varepsilon\left(\beta_{h}\right)}\right\rangle\right| \\
& \prod_{h=1}^{k^{\prime \prime \prime}}\left|\left\langle Q^{-} g_{\varepsilon\left(i_{h}^{\prime \prime \prime}\right)}, Q^{-} g_{1-\varepsilon\left(i_{h}^{\prime \prime \prime}\right)}\right\rangle_{\iota}\right| \cdot \prod_{h=1}^{m}\left|\left\langle Q^{-} S_{t_{p i}} g_{\varepsilon\left(p_{h}^{\prime \prime}\right)}, Q^{-} S_{t^{\prime \prime}} g_{1-\varepsilon\left(q_{h}^{\prime \prime}\right)}\right\rangle_{\iota}\right| \\
& \mid<W\left(z \int_{S / z^{2}}^{T / z^{2}} Q_{+} S_{u} f d u\right) \Phi_{F} \otimes W\left(z^{2} \int_{S / z^{2}}^{T / z^{2}} Q^{-} S_{u} f d u\right) \Phi_{F}^{\epsilon}, \\
& \left.W\left(z \int_{S^{\prime} / z^{2}}^{T^{\prime} / z^{2}} Q_{+} S_{u} f^{\prime} d u\right) \Phi_{F} \otimes W\left(z^{2} \int_{S^{\prime} / z^{2}}^{T^{\prime} / z^{2}} Q^{-} S_{u} f^{\prime} d u\right) \Phi_{F}^{\iota}\right\rangle \mid
\end{aligned}
$$

where, here and in the following,

$$
C_{1}:=\max _{F=f, f^{\prime} ; G=g_{0, g_{1} ; P=Q+, \ell \circ Q^{-}, I}} \int_{-\infty}^{\infty}\left|\left\langle P G, S_{t} P F\right\rangle\right| d t
$$

and

$$
\begin{gathered}
\bar{m}:=\left|\left\{q_{h}^{\prime}\right\}_{h=1}^{m^{\prime}} \cup\left\{q_{h}^{\prime}\right\}_{h=1}^{m^{\prime}}\right| \\
\left\{\beta_{h}\right\}_{h=1}^{\bar{m}}=\left\{q_{h}^{\prime}\right\}_{h=1}^{m^{\prime \prime}} \cup\left(\left\{q_{h}^{\prime \prime}\right\}_{h=1}^{m^{\prime \prime}} \backslash\left\{q_{h}^{\prime}\right\}_{h=1}^{m^{\prime}}\right) .
\end{gathered}
$$

Moreover, $\left\{\alpha_{h}\right\}_{h=1}^{\bar{m}}$ is chosen in the following way: 


$$
\alpha_{h}= \begin{cases}p_{h 0}^{\prime} & \text { if } \beta_{h}=q_{h 0}^{\prime} ; \\ p_{h 0}^{\prime \prime} & \text { if } \beta_{h}=q_{h 0}^{\prime \prime}\end{cases}
$$

With arguments similar to those of the proof of Lemma (3.1), one can get the following

Lemma (3.4). For each $n \in \mathbf{R}, \varepsilon \in\{0,1\}^{n}$,

$$
\begin{aligned}
& \mathrm{II} \varepsilon=\sum_{k=0}^{\varepsilon} \sum_{1 \leq i_{1}<\cdots<i_{k} \leq n} \sum_{k^{\prime}=0}^{n-k} \sum_{\substack{1 \leq i_{1}^{\prime}<\ldots<i_{k^{\prime}} \leq n \\
\left\{i_{h}\right\}_{h=1}^{k} \cap\left\{i_{h}^{\prime} k_{h=1}^{\prime}=\emptyset\right.}} \sum_{k^{\prime \prime}=1}^{n-k-k^{\prime}} \sum_{\substack{1 \leq i_{1}^{\prime \prime}<\cdots<i_{k}^{\prime \prime} \leq n \\
\left\{i_{k}^{\prime \prime} k_{h=1}^{\prime \prime} \cap\left(\left\{i_{h}\right\}_{h=1}^{k} \cup\left\{i_{h}^{\prime}\right\}_{h=1}^{k^{\prime}}\right)=\emptyset\right.}} \\
& \sum_{k^{\prime \prime \prime}=0}^{n-k-k^{\prime}} \sum_{\substack{\left.1 \leq i_{1}^{\prime \prime \prime}<\cdots<i_{k}^{\prime \prime \prime \prime \prime} \leq n \\
\left\{i i_{h}^{\prime \prime \prime}\right\}_{h=1}^{\prime \prime \prime}\right\} \cap\left(\left\{i h_{h=1}^{k} \cup\left\{i_{h}^{\prime} k_{h=1}^{k^{\prime}} \cup\left\{i_{h}^{\prime \prime \prime}\right\}_{h=1}^{k^{\prime \prime}}\right)=\emptyset\right.\right.}} \\
& \sum_{m=0}^{n-k-k^{\prime}-k^{\prime \prime \prime}} \sum_{1 \leq q_{1}<\cdots<q m \leq n} \sum_{m^{\prime}=0}^{k^{\prime} \wedge\left(n-k^{\prime}-k^{\prime \prime}-k^{\prime \prime \prime}-m\right)} \\
& \left\{q_{h}\right\}_{h=1}^{m} \subset\{1, \cdots, n\} \backslash\left(\left\{i_{h}\right\}_{h=1}^{k} \cup\left\{i_{h}^{\prime}\right\}_{h=1}^{k^{\prime}} \cup\left\{i_{h}^{\prime \prime}\right\}_{h=1}^{k^{\prime \prime}} \cup\left\{i_{h}^{\prime \prime \prime}\right\}_{h=1}^{k^{\prime \prime \prime}}\right)
\end{aligned}
$$

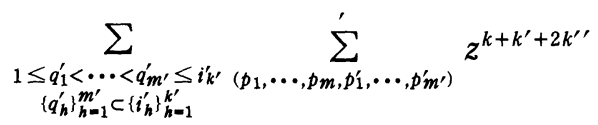

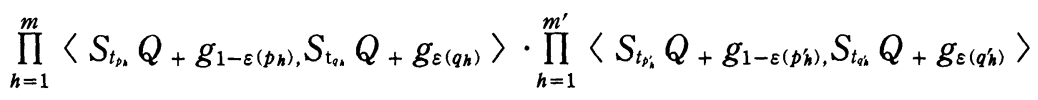

$$
\begin{aligned}
& \prod \quad A^{+}\left(S_{t_{\alpha}} Q+g_{\varepsilon(\alpha)}\right) \\
& \left.\alpha \in\{1, \cdots, n\} \backslash\left\{i_{h}\right\}_{h=1}^{k} \cup\left\{i_{h}^{\prime \prime}\right\}_{h=1}^{\prime \prime \prime} \cup\left\{i_{h}^{\prime \prime \prime}\right\}_{h=1}^{k \prime \prime} \cup\left\{q_{h}\right\}_{h=1}^{m} \cup\left\{q_{h}^{\prime}\right\}_{h=1}^{m^{\prime}}\right)
\end{aligned}
$$

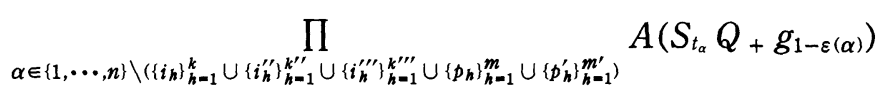

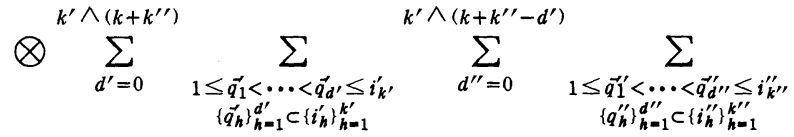

$$
\begin{aligned}
& \sum_{\left(\tilde{1}_{1}^{\prime}, \cdots, p_{d^{\prime}}^{\prime}, p_{1}^{\prime \prime}, \cdots, p_{d^{\prime \prime}}^{\prime \prime}\right)}^{\prime} \prod_{h=1}^{d^{\prime \prime \prime}}\left\langle Q^{-} S_{t_{p_{1}}} g_{\varepsilon\left(\tilde{p}_{h}^{\prime}\right)}, Q^{-} S_{t_{\alpha_{i}}} g_{1-\varepsilon\left(\vec{q}_{h}^{\prime}\right)}\right\rangle_{\iota} \\
& \prod_{h=1}^{d^{\prime \prime}}\left\langle Q-S_{t_{p \prime}} g_{\varepsilon\left(p_{h}^{\prime \prime}\right)}, Q-S_{t_{q \prime}^{\prime \prime}} g_{1-\varepsilon\left(\vec{q}_{h}^{\prime \prime}\right)}\right\rangle_{\iota} \\
& z^{2 k^{\prime \prime \prime}} \prod_{h=1}^{k^{\prime \prime \prime}}\left\langle Q^{-} g_{\varepsilon\left(i h^{\prime \prime}\right)}, Q^{-} g_{1-\varepsilon\left(i h^{\prime \prime}\right)}\right\rangle_{\iota} \\
& \prod_{\alpha \in\left(\left\{i_{h}^{\prime} k_{h=1}^{k^{\prime}} \cup\left\{i_{h}^{\prime \prime}\right\}_{h=1}^{k^{\prime \prime}} \backslash \backslash\left(q_{q_{h}^{\prime}}^{\prime}\right\}_{h=1}^{\prime \prime} \cup\left\{\vec{q}_{h}^{\prime}\right\}_{h=1}^{d^{\prime}}\right)\right.} A^{+}\left(Q-S_{t_{\alpha}} g_{1-\varepsilon(\alpha)}\right)
\end{aligned}
$$




$$
\prod_{\alpha \in\left(\left\{i_{h}^{\prime}\right\}_{h=1}^{k^{\prime}} \cup\left\{i_{h}^{\prime \prime}\right\}_{h=1}^{k^{\prime \prime}} \backslash \backslash\left(\left\{\vec{q}_{h}^{\prime}\right\}_{h=1}^{d^{\prime \prime}} \cup\left\{\vec{q}_{h}^{\prime}\right\}_{h-1}^{d^{\prime}}\right)\right.} A^{+}\left(Q^{-} S_{t_{\alpha}} g_{1-\varepsilon(\alpha)}\right)
$$

where, $\sum_{\left(p_{1}, \cdots, p_{m}, p_{1}^{\prime}, \cdots, p_{m^{\prime}}\right)}^{\prime}$ means the sum for all $1 \leq p_{1}, \cdots, p_{m}, p_{1}^{\prime}, \cdots, p_{m^{\prime}}^{\prime} \leq n$ which satisfying

(i) $\left\{p_{h}\right\}_{h=1}^{m} \cup\left\{p_{h}^{\prime}\right\}_{h=1}^{m^{\prime}} \subset\{1, \ldots, n\} \backslash\left(\left\{i_{h}^{\prime}\right\}_{h=1}^{k^{\prime}} \cup\left\{i_{h}^{\prime \prime}\right\}_{h=1}^{k^{\prime \prime}} \cup\left\{i_{h}^{\prime \prime \prime}\right\}_{h=1}^{k^{\prime \prime \prime}}\right)$;

(ii) $\left|\left\{p_{h}\right\}_{h=1}^{m} \cup\left\{p_{h}^{\prime}\right\}_{h=1}^{m^{\prime}}\right|=m+m^{\prime}$;

(iii) $p_{h}<q_{h}$ for all $h=1, \ldots, m$ and $p_{h}^{\prime}<q_{h}^{\prime}$ for all $h=1, \ldots, m^{\prime}$;

and $\sum_{\left(\bar{p}_{1}^{1}, \ldots, \bar{p}_{d^{\prime}}, \bar{p}_{1}^{\prime}, \ldots, \bar{p}_{d^{\prime}}^{\prime \prime}\right)}^{\prime}$, means the sum for all $1 \leq \bar{p}_{1}^{\prime}, \ldots, \bar{p}_{d^{\prime}}^{\prime}, \bar{p}_{1}^{\prime}, \ldots, \bar{p}_{d^{\prime \prime}}^{\prime \prime} \leq n$ satisfying

(i) $\left.\left\{\bar{p}_{h}^{\prime}\right\}_{h=1}^{d^{\prime}} \bigcup\left\{\bar{p}_{h}^{\prime \prime}\right\}_{h=1}^{d^{\prime \prime}} \subset\left\{i_{h}\right\}\right\}_{h=1}^{k} \bigcup\left\{i_{h}^{\prime \prime}\right\}_{h=1}^{k^{\prime \prime}}$;

(ii) $\left|\left\{\tilde{p}_{h}^{\prime}\right\}_{h=1}^{d^{\prime}} \cup\left\{\tilde{p}_{h}^{\prime \prime}\right\}_{h=1}^{d^{\prime \prime}}\right|=d^{\prime}+d^{\prime \prime}$;

(iii) $\bar{p}_{h}^{\prime}<\bar{q}_{h}^{\prime}$ for all $h=1, \ldots, d^{\prime}$ and $\bar{p}_{h}^{\prime \prime}<\bar{p}_{h}^{\prime \prime}$ for all $h=1, \ldots, d^{\prime \prime}$.

Corollary (3.5). For each $n \in \mathbf{N}, \varepsilon \in\{0,1\}^{n}, f, f^{\prime} \in K, T, S, T^{\prime}, S^{\prime} \in \mathbf{R}$,

$$
\begin{aligned}
& 1<W\left(z \int_{S / z^{2}}^{T / z^{2}} Q_{+} S_{u} f d u\right) \Phi_{F} \otimes W\left(z^{2} \int_{S / z^{2}}^{T / z^{2}} Q^{-} S_{u} f d u\right) \Phi_{F}^{\iota}, \\
& \int_{0}^{t^{/ 2} z^{2}} d t_{1} \int_{0}^{t_{1}} d t_{2} \cdots \int_{0}^{t_{n-1}} d t_{n} \mathrm{II}_{n}^{\varepsilon} \\
& \left.\cdot W\left(z \int_{S^{\prime} / z^{2}}^{T^{\prime} / z^{2}} Q_{+} S_{u} f^{\prime} d u\right) \Phi_{F} \otimes W\left(z^{2} \int_{S^{\prime} / z^{2}}^{T^{\prime} / z^{2}} Q^{-} S_{u} f^{\prime} d u\right) \Phi_{F}^{c}\right\rangle \mid
\end{aligned}
$$

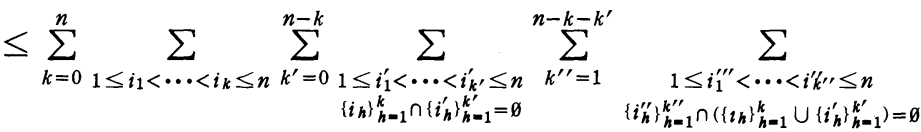

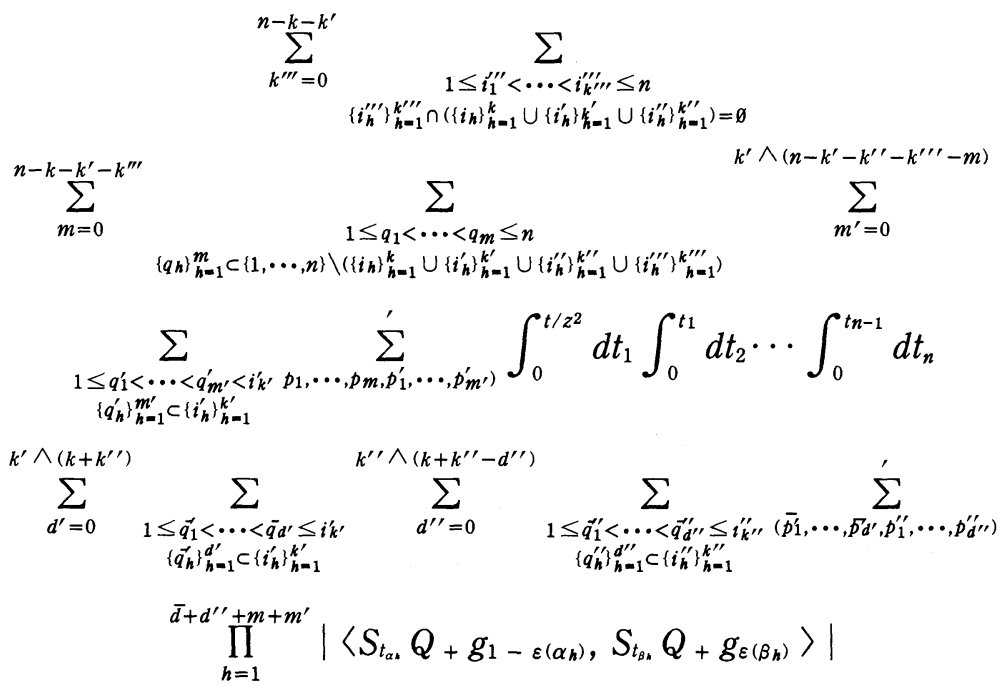




$$
\begin{aligned}
& C_{1}^{2\left(n-m-m^{\prime}-d^{\prime}-d^{\prime \prime}\right)} \cdot z^{2\left(n-m-m^{\prime}+2 k^{\prime \prime}+k+k^{\prime}-2 d^{\prime}-2 d^{\prime \prime}\right)} \\
& \prod_{h=1}^{k^{\prime \prime \prime \prime}}\left|\left\langle Q^{-} g_{1-\varepsilon\left(i h^{\prime \prime}\right)}, Q^{-} g_{\varepsilon\left(i h^{\prime \prime}\right)}\right\rangle\right| \cdot \prod_{h=1}^{d^{\prime}}\left|\left\langle Q^{-} S_{t_{\phi_{k}}} g_{\varepsilon\left(\bar{F}_{h}^{\prime}\right)}, Q^{-} S_{t_{\gamma_{h}}} g_{1-\varepsilon\left(\bar{p}_{h}^{\prime}\right)}\right\rangle_{c}\right| \\
& \left\langle W\left(z \int_{S / z^{2}}^{T / z^{2}} Q+S_{u} f d u\right) \Phi_{F} \otimes W\left(z^{2} \int_{S / z^{2}}^{T / z^{2}} Q-S_{u} f d u\right) \Phi_{F}^{\iota},\right. \\
& \left.W\left(z \int_{S^{\prime} / z^{2}}^{T^{\prime} / z^{2}} Q_{+} S_{u} f^{\prime} d u\right) \Phi_{F} \otimes W\left(z \int_{S^{\prime} / z^{2}}^{T^{\prime} / z^{2}} Q^{-} S_{u} f^{\prime} d u\right) \Phi_{F}^{<}\right\rangle \mid
\end{aligned}
$$

where,

$$
\begin{gathered}
\bar{d}:=\left|\left\{\bar{q}_{h}\right\}_{h=1}^{d^{\prime}} \backslash\left\{q_{h}^{\prime}\right\}_{h=1}^{m^{\prime}}\right| \\
\{\beta\}_{h=1}^{\bar{d}+d^{\prime \prime}+m+m^{\prime}}=\left\{q_{h}\right\}_{h=1}^{m} \bigcup\left\{q_{h}^{\prime}\right\}_{h=1}^{m^{\prime}} \bigcup\left\{\bar{p}_{h}^{\prime \prime}\right\}_{h=1}^{d^{\prime \prime}} \bigcup\left\{\bar{\beta}_{h}\right\}_{h=1}^{\bar{d}} \\
\left\{\bar{\beta}_{h}\right\}_{h=1}^{\bar{d}}:=\left\{\bar{q}_{h}^{\prime}\right\}_{h=1}^{d^{\prime}} \backslash\left\{q_{h}^{\prime}\right\}_{h=1}^{m^{\prime}} .
\end{gathered}
$$

with

\$4. The limit of the collective and the Negligible terms

In the section, we shall deal with the limits

$$
\begin{gathered}
\lim _{z \rightarrow 0}\left\langle W\left(z \int_{S / z^{2}}^{T / z^{2}} Q_{+} S_{u} f d u\right) \Phi_{F} \otimes W\left(z^{2} \int_{S / z^{2}}^{T / z^{2}} Q^{-} S_{u} f d u\right) \Phi_{F}^{\iota}\right. \\
\int_{0}^{t / z^{2}} d t_{1} \int_{0}^{t_{1}} d t_{2} \cdots \int_{0}^{t_{n-1}} d t_{n} I_{n}^{\varepsilon} \cdot \\
\left.\cdot W\left(z \int_{S^{\prime} / z^{2}}^{T^{\prime} / z^{2}} Q+S_{u} f^{\prime} d u\right) \Phi_{F} \otimes W\left(z^{2} \int_{S^{\prime} / z^{2}}^{T^{\prime} / z^{2}} Q^{-} S_{u} f^{\prime} d u\right) \Phi_{F}^{\iota}\right\rangle
\end{gathered}
$$

and

$$
\begin{gathered}
\lim _{z \rightarrow 0}\left\langle W\left(z \int_{S / z^{2}}^{T / z^{2}} Q_{+} S_{u} f d u\right) \Phi_{F} \otimes W\left(z^{2} \int_{S / z^{2}}^{T / z^{2}} Q^{-} S_{u} f d u\right) \Phi_{F}^{\iota},\right. \\
\int_{0}^{t / z^{2}} d t_{1} \int_{0}^{t_{1}} d t_{2} \cdots \int_{0}^{t n-1} d t_{n} I I_{n}^{\varepsilon} \cdot \\
\left.\cdot W\left(z \int_{S^{\prime} / z^{2}}^{T^{\prime} / z^{2}} Q+S_{u} f^{\prime} d u\right) \Phi_{F} \otimes W\left(z^{2} \int_{S^{\prime} / z^{2}}^{T^{\prime} / z^{2}} Q^{-} S_{u} f^{\prime} d u\right) \Phi_{F}^{\iota}\right\rangle
\end{gathered}
$$

for fixed $n \in \mathbf{N}$.

First of all, we have the following

Lemma (4.1). For each $n \in \mathbf{N}, 0 \leq m \leq n, 2 \leq q_{1}<\cdots<q_{m} \leq n, 1 \leq$ $p_{1}, \cdots, p_{m} \leq n-1, p_{h}<q_{h}, h=1, \ldots, m,\left\{f_{h}\right\}_{h=1}^{m} \subset L^{1}(\mathbf{R})$,

$$
z^{-2 m} \int_{0}^{t} d t_{1} \cdots \int_{0}^{t_{n-1}} d t_{n} \prod_{h=1}^{m}\left|f_{h}\left(\frac{t_{q_{h}}-t_{p_{h}}}{z^{2}}\right)\right| \leq \frac{t^{n-m}}{(n-m) !} \prod_{h=1}^{m} \int_{-\infty}^{\infty}\left|f_{h}(t)\right| d t
$$


as $z \rightarrow 0$.

The proof is similar to that of the lemma (3.3) of [1].

Lemma (4.2). For each $n \in \mathbf{N}, 0 \leq m \leq n, f, g \in K, 2 \leq q_{1}<\cdots<q_{m}$ $\leq n, 1 \leq p_{1}, \ldots, p_{m} \leq n-1, p_{h}<q_{h}, h=1, \ldots, m$,

$$
z^{-2 m} \int_{0}^{t} d t_{1} \cdots \int_{0}^{t_{n-1}} d t_{n} \prod_{h=1}^{m}\left|\left\langle Q_{+} f, S_{\frac{t_{\alpha}-t_{n}}{z^{2}}} Q+g\right\rangle\right|=O(1)
$$

as $z \rightarrow 0$.

Remark. Lemma (4.2) is not a direct corollary of Lemma (4.1) because in (4.4), the operator $Q_{+}$depends on $\boldsymbol{z}$.

Proof. By the definition, one has

So,

$$
Q_{+}^{2}=\left(1+z^{2} e^{-\frac{1}{2} \beta H}\right)^{-1}=\sum_{p=0}^{\infty} z^{2 p} e^{-\frac{1}{2} \phi \beta H} .
$$

$$
\begin{gathered}
z^{-2 m} \int_{0}^{t} d t_{1} \cdots \int_{0}^{t_{n-1}} d t_{n} \prod_{n=1}^{m}\left|\left\langle Q_{+} f, S_{\frac{t_{u}-t_{\mu_{\mu}}}{z^{2}}} Q_{+} g\right\rangle\right| \\
\leq \sum_{p=0}^{\infty} z^{2 p} \cdot z^{-2 m} \int_{0}^{t} d t_{1} \cdots \int_{0}^{t n-1} d t_{n} \prod_{h=1}^{m}\left|\left\langle f, S_{\frac{t_{\alpha}-t_{\alpha}}{z^{2}}} e^{-\frac{1}{2} p \beta H} g\right\rangle\right| .
\end{gathered}
$$

Applying Lemma (4.1) to (4.5), one finds a majorization of the left hand side of (4.5) by

$$
\sum_{p=0}^{\infty} z^{2 p} \cdot \frac{t^{n-m}}{(n-m) !}\left(\int_{-\infty}^{\infty}\left|\left\langle f, S_{t} e^{-\frac{1}{2} p \beta H} g\right\rangle\right| d t\right)^{m}
$$

Combining together this and the fact that if the series

$$
\sum_{n=1}^{\infty} z^{n} a_{n}, a_{n} \geq 0, n=1,2, \ldots
$$

has a positive convergence radius, then for any $m \in \mathbf{N}$, the series

$$
\sum_{n=1}^{\infty} z^{n}\left(a_{n}\right)^{m}, a_{n} \geq 0, n=1,2, \ldots
$$

also has a positive convergence radius, the proof is ended.

THEOREM (4.3). For each $n \in \mathbf{N}$,

$$
\begin{gathered}
\lim _{z \rightarrow 0}\left\langle W\left(z \int_{S / z^{2}}^{T / z^{2}} Q+S_{u} f d u\right) \Phi_{F} \otimes W\left(z^{2} \int_{S / z^{2}}^{T / z^{2}} Q^{-} S_{u} f d u\right) \Phi_{F}^{\iota}\right. \\
\int_{0}^{t / z^{2}} d t_{1} \int_{0}^{t_{1}} d t_{2} \cdots \int_{0}^{t_{n-1}} d t_{n} \mathrm{II}_{n}^{\varepsilon} .
\end{gathered}
$$




$$
\left.\cdot W\left(z \int_{S^{\prime} / z^{2}}^{T^{\prime} / z^{2}} Q+S_{u} f^{\prime} d u\right) \Phi_{F} \otimes W\left(z \int_{S^{\prime} / z^{2}}^{T^{\prime} / z^{2}} Q^{-} S_{u} f^{\prime} d u\right) \Phi_{F}^{\iota}\right\rangle=0
$$

Proof. In (3.21), one has

$$
\begin{gathered}
z^{2\left(n+k+k^{\prime}+2 k^{\prime \prime}-2 d^{\prime}-2 d^{\prime \prime}-m-m^{\prime}\right)} \int_{0}^{t / z^{2}} d t_{1} \int_{0}^{t_{1}} d t_{2} \cdots \int_{0}^{t_{n-1}} d t_{n} \\
\prod_{h=1}^{\bar{d}+d^{\prime \prime}+m+m^{\prime}}\left|\left\langle S_{t_{\alpha}} Q+g_{\varepsilon\left(\alpha_{h}\right)}, S_{t_{\beta_{k}}} Q+g_{1-\varepsilon\left(\beta_{h}\right)}\right\rangle\right| \\
\leq z^{-2\left(m+m^{\prime}+\bar{d}+d^{\prime \prime}\right)} \cdot z^{2\left(k+k^{\prime}+2 k^{\prime \prime}-\bar{d}-d^{\prime}\right)} \int_{0}^{t} d t_{1} \int_{0}^{t_{1}} d t_{2} \cdots \int_{0}^{t_{n-1}} d t_{n} \\
\prod_{h=1}^{\bar{d}+d^{\prime \prime}+m+m^{\prime}}\left|\left\langle Q+g_{\varepsilon\left(\alpha_{h}\right),} S_{\frac{t_{\beta_{n}} t_{\alpha_{k}}}{z^{2}}} Q+g_{1-\varepsilon\left(\beta_{h}\right)}\right\rangle\right| .
\end{gathered}
$$

By definition, $1 \leq \beta_{1}<\cdots<\beta_{\bar{d}+d^{\prime \prime}+m+m^{\prime}} \leq \boldsymbol{n}$, so applying Lemma (4.2) to (4.7), one gets

$$
\begin{gathered}
z^{-2\left(m+m^{\prime}+\bar{d}+d^{\prime \prime}\right)} \cdot z^{2\left(k+k^{\prime}+2 k^{\prime \prime}+\bar{d}-d^{\prime}\right)} \int_{0}^{t} d t_{1} \int_{0}^{t_{1}} d t_{2} \cdots \int_{0}^{t_{n-1}} d t_{n} \\
\prod_{h=1}^{\bar{d}+d^{\prime \prime}+m+m^{\prime}}\left|\left\langle Q_{+} g_{\varepsilon\left(\alpha_{h}\right)}, S_{\frac{t_{\theta_{1}}-t_{\alpha_{1}}}{z^{2}}} Q_{+} g_{1-\varepsilon\left(\beta_{h}\right)}\right\rangle\right| \\
\leq z^{2\left(k^{\prime}+k^{\prime \prime}+\bar{d}-d^{\prime}\right)} \cdot O(1) .
\end{gathered}
$$

Notice that $d^{\prime} \leq k^{\prime}$ and in $\mathrm{II}_{n}^{\varepsilon}, k^{\prime \prime} \geq 1$, so, the right hand side of (4.8) goes to zero as $z \rightarrow 0$.

In the following we shall consider the limit of the right hand side of (3.14). The first step is to show that we need only to consider the situation in which there are the same number of $A_{-1}$ and $A_{1}$, i.e. $k=k^{\prime}$.

THEOREM (4.4). In the right hand side of (3.15), if $k \neq k^{\prime}$, then

$$
\begin{gathered}
\lim _{z \rightarrow 0} z^{2\left(n+k+k^{\prime}-m^{\prime}-m-2 m^{\prime \prime}\right)} \cdot \int_{0}^{t^{\prime} z^{2}} d t_{1} \int_{0}^{t_{1}} d t_{2} \cdots \int_{0}^{t_{n-1}} d t_{n} \\
\prod_{h=1}^{m}\left|\left\langle S_{t_{\phi_{n}}} Q_{+} g_{1-\varepsilon\left(p_{h}\right),} S_{t_{t_{\alpha}}} Q_{+} g_{\varepsilon\left(q_{h}\right)}\right\rangle\right| \cdot \prod_{h=1}^{\bar{m}}\left|\left\langle S_{t_{\phi_{k}}} Q_{+} g_{1-\varepsilon\left(\alpha_{h}\right),}, S_{t_{\beta_{k}}} Q_{+} g_{\varepsilon\left(\beta_{h}\right)}\right\rangle\right|=0 .
\end{gathered}
$$

Proof. With the change of variables

$$
z^{2} t_{j} \hookrightarrow t_{j}, j=1, \ldots, n
$$

one has

$$
z^{2\left(n+k+k^{\prime}-m^{\prime}-m-2 m^{\prime \prime}\right)} \cdot \int_{0}^{t / z^{2}} d t_{1} \int_{0}^{t_{1}} d t_{2} \cdots \int_{0}^{t_{n-1}} d t_{n}
$$




$$
\begin{aligned}
& \prod_{h=1}^{m}\left|\left\langle S_{t_{p^{\prime}}} Q+g_{1-\varepsilon\left(p_{h}\right)}, S_{t_{t^{\prime}}} Q+g_{\varepsilon\left(q_{h}\right)}\right\rangle\right| \cdot \prod_{h=1}^{\bar{m}}\left|\left\langle S_{t_{\alpha}} Q+g_{1-\varepsilon\left(\alpha_{h}\right)}, S_{t_{\beta^{\prime}}} Q+g_{\varepsilon\left(\beta_{h}\right)}\right\rangle\right| \\
& =z^{-2(\bar{m}+m)} \cdot z^{2\left(k+k^{\prime}-m^{\prime}+\bar{m}-2 m^{\prime \prime}\right)} \cdot \int_{0}^{t} d t_{1} \int_{0}^{t_{1}} d t_{2} \cdots \int_{0}^{t_{n-1}} d t_{n} \\
& \prod_{h=1}^{m}\left|\left\langle Q+g_{1-\varepsilon\left(p_{h}\right)}, S_{\frac{t_{\alpha_{h}-t_{h}}}{z^{2}}} Q+g_{\varepsilon\left(q_{h}\right)}\right\rangle\right| \cdot \prod_{h=1}^{\bar{m}}\left|\left\langle Q+g_{1-\varepsilon\left(\alpha_{h}\right)}, \quad S_{\frac{t_{\beta_{h}-t_{\alpha}}}{z^{2}}} Q+g_{\varepsilon\left(\beta_{h}\right)}\right\rangle\right| .
\end{aligned}
$$

By the defininion one has $\left\{\beta_{h}\right\}_{h=1}^{\bar{m}} \cap\left\{q_{h}\right\}_{h=1}^{m}=\emptyset$, therefore

$$
\begin{aligned}
& z^{-2(\bar{m}+m)} \cdot \int_{0}^{t} d t_{1} \int_{0}^{t_{1}} d t_{2} \cdots \int_{0}^{t_{n-1}} d t_{n} \prod_{h=1}^{m}\left|\left\langle Q+g_{1-\varepsilon\left(p_{h}\right)}, S_{\frac{t_{v_{n}} t_{p_{n}}}{z^{2}}} Q_{+} g_{\varepsilon\left(q_{h}\right)}\right\rangle\right| \\
& \prod_{h=1}^{\bar{m}}\left|\left\langle Q+g_{1-\varepsilon\left(\alpha_{h}\right)}, S_{\frac{t_{\beta_{h}}-t_{\alpha_{\alpha}}}{z^{2}}} Q+g_{\varepsilon\left(\beta_{h}\right)}\right\rangle\right|=O(1) .
\end{aligned}
$$

By the definition (3.17), we obtain that

$$
\bar{m} \geq m^{\prime}
$$

so,

$$
k+k^{\prime}+\bar{m}-m^{\prime}-2 m^{\prime \prime} \geq k+k^{\prime}-2 m^{\prime \prime} .
$$

If $k \neq k^{\prime}$, then $2 m^{\prime \prime} \leq 2\left(k \wedge k^{\prime}\right)<k+k^{\prime}$ and this implies that the right hand side of (4.10) is less or equal to

$$
z^{k+k^{\prime}-2 m^{\prime \prime}} \cdot O(1) \leq z \cdot O(1)
$$

and goes to 0 as $z \rightarrow 0$.

Our second step is to show that with the condition $k=k^{\prime}$, one needs only to consider the situation in which the projection on the conjugate Fock space of all $A_{ \pm 1}$ are used to produce scalar products in the normally ordered form, i.e. in the notations of Corollary (3.2), $m^{\prime \prime}=k\left(=k^{\prime}\right)$.

COROLlary (4.5). If in the right hand side of (3.15), $k=k^{\prime}$ and $m^{\prime \prime}<k$, then (4.9) is true also.

Proof. In the present case, by repeating the proof of Theorem (4.4), one finds a majorization of the quantity in the left hand side of (4.9) by

$$
z^{k+k^{\prime}-2 m^{\prime}} \cdot O(1)=z^{2} \cdot O(1)
$$

this ends the proof.

Theorem (4.4) and Corollary (4.5) show that in the following we need only to consider the limit of 


$$
\begin{aligned}
& I_{n}^{\varepsilon}(1, z, t):=\sum_{k=0}^{[n / 2]} \sum_{1 \leq i_{1}<\cdots<i_{k} \leq n} \sum_{1 \leq i_{1}^{\prime}<\cdots<i_{k}^{\prime} \leq n} \\
& \left\{i_{h}\right\}_{h=1}^{k} \cap\left\langle i_{h}^{\prime}\right\}_{h=1}^{k}=\emptyset ; i_{h}<i_{h}^{\prime}, h=1, \cdots, k \\
& \sum_{k^{\prime \prime \prime}=0}^{n-2 k} \sum_{\substack{1 \leq i_{1}^{\prime \prime}<\cdots<i_{k}^{\prime \prime \prime \prime} \leq n \\
k^{\prime \prime \prime \prime}}} \\
& \left\{i_{h}^{\prime \prime \prime}\right\}_{h=1}^{k \prime \prime \prime} \cap\left(\{i h\}_{h=1}^{k} \cup\left\{i_{h}^{\prime}\right\}_{h=1}^{k}\right)=\varnothing
\end{aligned}
$$

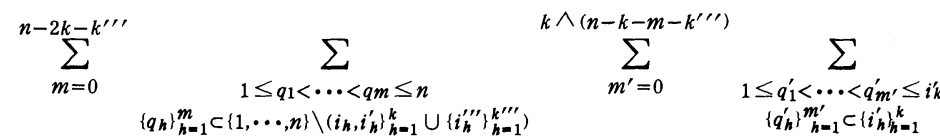

$$
\begin{aligned}
& \sum_{\left(p_{1}, \cdots, p_{m}, p_{1}^{\prime}, \cdots, p^{\prime} m^{\prime}\right)} \\
& z^{2\left(n-m^{\prime}-m\right)} \cdot \int_{0}^{t / z^{2}} d t_{1} \int_{0}^{t_{1}} d t_{2} \cdots \int_{0}^{t n-1} d t_{n}
\end{aligned}
$$

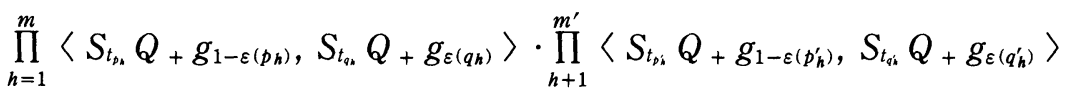

$$
\begin{aligned}
& \prod_{\alpha \in\{1, \cdots, n\} \backslash\left(i_{h}, i_{h}^{\prime}\right\}_{h=1}^{k} \cup\left\{q_{h}\right\}_{h=1}^{m}} \int_{S / z^{2}}^{T / z^{2}} d u\left\langle S_{u} Q_{+} f, S_{t_{\alpha}} Q_{+} g_{\varepsilon(\alpha)}\right\rangle \\
& \prod_{\alpha \in\left\{i_{h}^{\prime}\right\}_{h=1}^{k} \backslash\left\{q_{h}^{\prime} h_{h=1}^{m^{\prime}}\right.} \int_{S / z^{2}}^{T / z^{2}} d u\left\langle S_{u} Q_{+} f, S_{t_{\alpha}} Q_{+} g_{\varepsilon(\alpha)}\right\rangle \\
& \prod_{\alpha \in\left\{1, \cdots, n \nmid \backslash\left(i_{h}^{\prime}\right\}_{h=1}^{k} \cup\left\{p_{h}\right\}_{h=1}^{m} \cup\left\{p_{h}^{\prime}\right\}_{h=1}^{m^{\prime}}\right)} \int_{S / z^{2}}^{T / z^{2}} d u\left\langle S_{t_{\alpha}} Q+g_{1-\varepsilon(\alpha)}, S_{u} Q+f^{\prime}\right\rangle \\
& \left.\sum_{\sigma \in S_{k}^{\prime}} \prod_{h+1}^{k}\left\langle Q^{-} S_{t_{\sigma_{h}}} g_{\varepsilon\left(t_{o}\right)}, Q^{-} S_{t_{i}} g_{1-\varepsilon\left(i_{h}^{\prime}\right)}\right)\right\rangle_{\iota} \cdot \prod_{h=1}^{\mathrm{k}^{\prime \prime \prime \prime}}\left\langle Q^{-} g_{\varepsilon\left(i_{h}^{\prime \prime \prime}\right)}, Q^{-} g_{1-\varepsilon\left(i_{h}^{\prime \prime \prime}\right)}\right\rangle_{\iota} \\
& \left\langle W\left(z \int_{S / z^{2}}^{T / z^{2}} Q+S_{u} f d u\right) \Phi_{F} \otimes W\left(z^{2} \int_{S / z^{2}}^{T / z^{2}} Q^{-} S_{u} f d u\right) \Phi_{F}^{\prime},\right. \\
& \left.W\left(z \int_{S^{\prime} / z^{2}}^{T^{\prime} / z^{2}} Q+S_{u} f^{\prime} d u\right) \Phi_{F} \otimes W\left(z^{2} \int_{S^{\prime} / z^{2}}^{T^{\prime} / z^{2}} Q^{-} S_{u} f^{\prime} d u\right) \Phi_{F}^{\prime}\right\rangle
\end{aligned}
$$

where, for each $\left\{i_{h}, i_{h}^{\prime}\right\}_{h=1}^{k}, i_{h}<i_{h}^{\prime}, h=1, \ldots, k$,

$$
S_{k}^{\prime}:=\left\{\sigma \in S_{k}: i_{\sigma(h)}<i_{h}^{\prime}, h=1, \ldots, k\right\} .
$$

Remark. In the sum $\sum_{\substack{1 \leqslant i_{i}<\ldots<i_{i} \leq n \\\left\langle i_{h}\right\}_{h=1}^{k} \backslash\left\{i_{h}^{\prime}\right\}_{h=1}^{\prime}=\emptyset ; i_{n}<i_{n}, h=1, \ldots, k}}$, the conditions $i_{h}<i_{h}^{\prime}, h=$ $1, \ldots, k$ dues to on the conjugate space, all operators are used to produce scalar products $\left(m^{\prime \prime}=k\right)$, so all creators should be to the right hand side of annihila- 
tors.

Stating from (4.14), our third step is to show that those terms in the right hand side of (4.14) in which $m^{\prime \prime}<k$ tend to zero, i.e. one needs only to consider the terms in which the projection on the Fock space (not only the conjugate one) of all $A_{-1}$ and $A_{1}$ are used to produce scalar products in the normally ordered form.

THEOREM (4.6). In the right hand side of (4.14), if $m^{\prime \prime}<k$, then the quantity

$$
\begin{aligned}
& z^{2\left(n-m-m^{\prime}\right)} \cdot \int_{0}^{t / z^{2}} d t_{1} \int_{0}^{t_{1}} d t_{2} \cdots \int_{0}^{t_{n-1}} d t_{n} \\
& \prod_{h=1}^{m}\left|\left\langle S_{t_{p h}} Q+g_{1-\varepsilon\left(p_{h}\right)}, S_{t_{\phi,}} Q+g_{\varepsilon\left(q_{h}\right)}\right\rangle\right| \cdot \prod_{h=1}^{m^{\prime}}\left|\left\langle S_{t_{p i}} Q+g_{1-\varepsilon\left(p_{h}^{\prime}\right)}, S_{t_{\phi k}} Q+g_{\varepsilon\left(q_{h}^{\prime}\right)}\right\rangle\right| \\
& \prod_{h=1}^{k}\left|\left\langle Q^{-} S_{t_{i_{h}}} g_{\varepsilon\left(i_{\sigma_{k}}\right)}, Q^{-} S_{t_{i, h}} g_{1-\varepsilon\left(i_{h}^{\prime}\right)}\right\rangle\right|
\end{aligned}
$$

tends to zero as $z \rightarrow 0$.

Proof. With the change of variables (4.10a) and since the product

$$
\prod_{h=1}^{m^{\prime}}\left|\left\langle S_{t_{p i}} Q+g_{1-\varepsilon\left(p_{h}^{\prime}\right)}, S_{t_{\phi_{i}}} Q_{+} g_{\varepsilon\left(q_{h}^{\prime}\right)}\right\rangle\right|
$$

is less than or equal to

$$
\left(2\left\|g_{0}\right\| \cdot\left\|g_{1}\right\|\right)^{m^{\prime}}
$$

we know that the left side of (4.16) is equal to

$$
\begin{aligned}
& z^{2\left(k-m^{\prime}\right)} \cdot z^{-2(k+m)} \cdot \int_{0}^{t} d t_{1} \int_{0}^{t_{1}} d t_{2} \cdots \int_{0}^{t n-1} d t_{n} \prod_{h=1}^{m}\left|\left\langle Q+g_{1-\varepsilon\left(p_{h}\right)}, S_{\frac{t_{u-\alpha}-t_{2}}{z^{2}}} Q_{+} g_{\varepsilon\left(q_{h}\right)}\right\rangle\right| \\
& \prod_{h=1}^{k} \mid\left\langle\iota Q^{-} g_{\varepsilon\left(i_{a_{0}}\right)}, S_{\frac{t_{t_{0}-t_{\text {ias }}}}{2}} \iota Q^{-} g_{1-\varepsilon\left(i_{h}^{\prime}\right)}>\right| \cdot O(1) .
\end{aligned}
$$

Notice that the sets of indices $\left\{q_{h}\right\}_{h=1}^{m}$ and $\left\{i_{h}\right\}_{h=1}^{k}$ are disjoint and by (4.2), the quantity (4.18) becomes to

$$
z^{2\left(k-m^{\prime}\right)} \cdot O(1)
$$

and it tends to zero if $m^{\prime}<k$.

Remark. The proof of Theorem (4.6) can not give the conclusion that $m=k$ because $\left\{q_{h}^{\prime}\right\}_{h=1}^{m^{\prime}} \subset\left\{i_{h}^{\prime}\right\}_{h=1}^{k}$ are NOT disjoint.

Theorem (4.6) shows that $\left\{q_{h}^{\prime}\right\}_{h=1}^{m^{\prime}}=\left\{i_{h}\right\}_{h=1}^{k}$ therefore

$$
\lim _{z \rightarrow 0} \mathrm{I}_{n}^{\varepsilon}(1, z, t)=\lim _{z \rightarrow 0} \mathrm{I}_{n}^{\varepsilon}(2, z, t)
$$




$$
\begin{aligned}
& :=\lim _{z \rightarrow 0} \sum_{k=0}^{[n / 2]} \sum_{1 \leq i_{1}<\cdots<i_{k} \leq n} \sum_{1 \leq i_{1}^{\prime}<\cdots<i_{k^{\prime}} \leq n} \\
& \left\{i_{h}\right\}_{h=1}^{k} \cap\left\{i_{h}^{\prime}\right\}_{h=1}^{k}=\emptyset ; i_{h}<i_{h}^{\prime}, h=1, \cdots, k \\
& \sum_{k^{\prime \prime \prime}=0}^{n-2 k} \sum_{\substack{1 \leq i_{1}^{\prime \prime}<\cdots<i^{\prime \prime \prime \prime \prime} \leq n\\
}} \\
& \left\{i_{h}^{\prime \prime \prime}\right\}_{h=1}^{k \prime \prime} \cap\left(\left\{i h_{h=1}^{k} \cup\left\langle i_{h}^{\prime}\right\}_{h=1}^{k}\right)=\emptyset\right. \\
& \sum_{m=0}^{n-2 k-k^{\prime \prime \prime}} \sum_{\substack{1 \leq q_{1}<\cdots<q_{m} \leq n \\
\left\{q_{h}\right\}_{h=1}^{m} \subset\{1, \cdots, n\} \backslash\left(\left\{i_{h}, i_{h}^{\prime}\right\}_{h=1}^{k} \cup\left\{i_{h}^{\prime \prime \prime}\right\}_{h=1}^{\left.k^{\prime \prime \prime}\right)}\right.}} \sum_{\left.\left(p_{1}, \cdots, p_{m}, p_{1}^{\prime}, \cdots, p_{h}^{\prime},\left\{i_{h}^{\prime}\right\}_{h=1}^{k}\right)^{k}\right)} \\
& z^{2(n-k-m)} \cdot \int_{0}^{t / z^{2}} d t_{1} \int_{0}^{t_{1}} d t_{2} \cdots \int_{0}^{t n-1} d t_{n}
\end{aligned}
$$

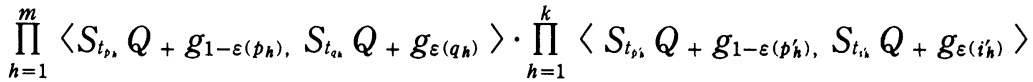

$$
\begin{aligned}
& \prod_{\left.\alpha \in\{1, \cdots, n\} \backslash\left\{i_{h}, i_{h}^{\prime}\right\}_{h=1}^{k} \cup\left\{i_{h}^{\prime \prime \prime}\right\}_{h=1}^{\prime \prime \prime \prime} \cup\left\{q_{h}\right\rangle_{h=1}^{m}\right)} \int_{S / z^{2}}^{T / z^{2}} d u\left\langle S_{u} Q+f, S_{t_{\alpha}} Q+g_{\varepsilon(\alpha)}\right\rangle \\
& \prod_{\left.\alpha \in\left\{i^{\prime}\right\}^{\prime}\right\}_{h=1}^{k} \backslash\left\{q_{h}^{\prime}\right\}_{h=1}^{m^{\prime}}} \int_{S / z^{2}}^{T / z^{2}} d u\left\langle S_{u} Q+f, S_{t_{\alpha}} Q+g_{\varepsilon(\alpha)}\right\rangle \\
& \prod_{\alpha \in\left\{1, \cdots, n \nmid \backslash\left\langlei _ { h } ^ { \prime } h _ { h = 1 } ^ { k } \cup \{ i _ { h } ^ { \prime \prime \prime } \} _ { h = 1 } ^ { k ^ { \prime \prime \prime } \cup } \cup \{ p _ { h } \} _ { h = 1 } ^ { m } \left\{ p_{h}^{\prime} m_{h=1}^{\left.m^{\prime}\right)}\right.\right.\right.} \int_{S / z^{2}}^{T / z^{2}} d u\left\langle S_{t_{a}} Q+g_{1-\varepsilon(\alpha),} S_{u} Q+f^{\prime}\right\rangle . \\
& \sum_{\sigma \in S^{\prime} k} \prod_{h=1}^{k}\left\langle Q-S_{t_{i h_{h}}} g_{\varepsilon\left(i_{o}\right)}, Q^{-} S_{t_{i h}} g_{1-\varepsilon\left(i_{h}^{\prime}\right)}\right\rangle_{c} \\
& \prod_{h=1}^{k^{\prime \prime \prime}}\left\langle Q^{-} g_{\varepsilon\left(i k^{\prime \prime}\right)}, Q^{-} g_{1-\varepsilon\left(i h^{\prime \prime}\right)}\right\rangle_{\iota} \\
& \left\langle W\left(z \int_{S / z^{2}}^{T / z^{2}} Q+S_{u} f d u\right) \Phi_{F} \otimes W\left(z^{2} \int_{S / z^{2}}^{T / z^{2}} Q^{-} S_{u} f d u\right) \Phi_{F}^{\epsilon},\right. \\
& \left.W\left(z \int_{S^{\prime} / z^{2}}^{T^{\prime} / z^{2}} Q_{+} S_{u} f^{\prime} d u\right) \Phi_{F} \otimes W\left(z^{2} \int_{S^{\prime} / z^{2}}^{T^{\prime} / z^{2}} Q^{-} S_{u} f^{\prime} d u\right) \Phi_{F}^{\iota}\right\rangle
\end{aligned}
$$

where, $\sum_{\left(p_{1}, \cdots, p_{m}, p_{1}^{\prime}, \cdots, p_{k}^{\prime},\left\{i_{h}^{\prime}\right\}_{h=1}^{k}\right)}$ means the sum for all $1 \leq p_{1}, \cdots, p_{m}, p_{1}^{\prime}, \cdots, p_{k}^{\prime} \leq n$ which satisfies

(i) $\left\{p_{h}\right\}_{h=1}^{m} \cup\left\{p_{h}^{\prime}\right\}_{h=1}^{k} \subset\{1, \ldots, n\} \backslash\left(\left\{i_{h}^{\prime}\right\}_{h=1}^{k} \bigcup\left\{i_{h}^{\prime \prime \prime}\right\}_{h=1}^{k^{\prime \prime \prime}}\right)$;

(ii) $\left|\left\{p_{h}\right\}_{h=1}^{m} \cup\left\{p_{h}^{\prime}\right\}_{h=1}^{k}\right|=m+k$;

(iii) $p_{h}<q_{h}$ for all $h=1, \ldots, m$ and $p_{h}^{\prime}<i_{h}^{\prime}$ for all $h=1, \ldots, k$.

Our fourth step is to show that we need only to investigate the situation in which

$$
p_{h}=q_{h}-1, h=1, \ldots, m
$$


and

$$
p_{h}^{\prime}=i_{h}^{\prime}-1, h=1, \ldots, k
$$

THEOREM (4.7). For each $m, k$, those terms of right hand side of (4.19) in which either (4.20a) or (4.20b) does not hold will tend to zero as $z \rightarrow 0$.

Proof. We should prove that if there exists a $h=1, \ldots, m$, such that $q_{h}$ $p_{h} \geq 2$ or a $h=1, \ldots, k$, such that $i_{h}^{\prime}-p_{h}^{\prime} \geq 2$, then,

$$
z^{2(n-k-m)} \cdot \int_{0}^{t / z^{2}} d t_{1} \int_{0}^{t_{1}} d t_{2} \cdots \int_{0}^{t_{n-1}} d t_{n}
$$

$$
\prod_{h=1}^{m}\left|\left\langle S_{t_{s,}} Q+g_{1-\varepsilon\left(p_{h}\right)}, S_{t_{\alpha,}} Q+g_{\varepsilon\left(q_{h}\right)}\right\rangle\right| \cdot \prod_{h=1}^{k}\left|\left\langle S_{t_{s,}} Q+g_{1-\varepsilon\left(p_{h}^{\prime}\right),} S_{t_{i s}} Q_{+} g_{\varepsilon\left(i^{\prime}\right)}\right\rangle\right| \longrightarrow 0 .
$$

The fact $\left\{q_{h}\right\}_{h=1}^{m} \cap\left\{i_{h}\right\}_{h=1}^{k}=\varnothing$ implies that the function in (4.21) is made of type II terms in the sense of [1] and therefore the same arguments as those of the proof of Lemma (3.3) imply the Theorem.

Theorem (4.7) shows that

$$
\begin{aligned}
& \lim _{z \rightarrow 0} \mathrm{I}_{n}^{\varepsilon}(1, z, t)=\lim _{z \rightarrow 0} \mathrm{I}_{n}^{\varepsilon}(2, z, t)=\lim _{z \rightarrow 0} \mathrm{I}_{n}^{\varepsilon}(3, z, t) \\
& :=\lim _{z \rightarrow 0} \sum_{k=0}^{[n / 2]} \sum_{1 \leq i_{1}<\cdots<i_{k} \leq n} \sum_{1 \leq i_{1}^{\prime}<\cdots<i_{k}^{\prime} \leq n} \\
& \left\{i_{h}\right\}_{h=1}^{k} \cap\left\{i_{h}^{\prime}\right\}_{h=1}^{k}=\emptyset ;\left\{i_{h}^{\prime}-1\right\}_{h=1}^{k} \cap\left\{i_{h}^{\prime}\right\}_{h=1}^{k}=\emptyset ; i_{h}<i_{h}^{\prime}, h=1, \cdots, k \\
& \sum_{k^{\prime \prime \prime}=0}^{n-2 k} \sum_{1 \leq i_{1}^{\prime \prime \prime}<\cdots<i_{k}^{\prime \prime \prime \prime \prime} \leq n} \\
& \left.\left.\left\{i_{h}^{\prime \prime \prime}\right\}_{h=1}^{k^{\prime \prime \prime}} \cap\left\{i_{h}\right\}\right\}_{h=1}^{\prime} \cup\left\{i_{h}^{\prime}\right\}_{h=1}^{k}\right)=\varnothing \\
& \sum_{m=0}^{n-2 k-k^{\prime \prime \prime}} \\
& \sum_{1 \leq q_{1}<\cdots<q_{m} \leq n} \\
& \left\{q_{h}\right\}_{h=1}^{m} \subset\{1, \cdots, n\rangle \backslash\left\langle\left\{i_{h}, i_{h}^{\prime}\right\}_{h=1}^{k} \cup\left\{i_{h}^{\prime \prime \prime}\right\}_{h=1}^{k^{\prime \prime \prime}}\right) ;\left\{q_{h}-1\right\}_{h=1}^{m} \cap\left(\left\langle i_{h}^{\prime}, i_{h}^{\prime}-1\right\}_{h=1}^{k} \cup{ }_{h=1}^{k^{\prime \prime \prime}}\right)=\varnothing \\
& z^{2(n-k-m)} \cdot \int_{0}^{t / z^{2}} d t_{1} \int_{0}^{t_{1}} d t_{2} \cdots \int_{0}^{t_{n-1}} d t_{n} \\
& \sum_{\sigma \in S_{k}^{\prime}} \prod_{h=1}^{k}\left\langle Q^{-} S_{t_{i_{h}}} g_{\varepsilon\left(t_{o_{k}}\right)}, Q^{-} S_{t_{\mathrm{i}}} g_{1-\varepsilon\left(t_{i}\right)}\right\rangle_{c}
\end{aligned}
$$

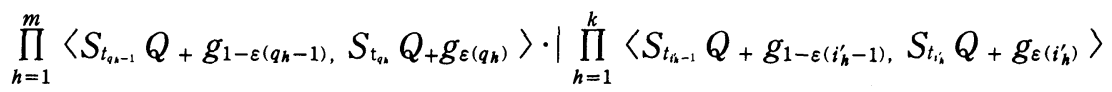

$$
\begin{aligned}
& \prod_{\alpha \in\left\{1, \cdots, n \backslash \backslash\left\{i_{h}, i_{h}^{\prime}\right\}_{h=1}^{k} \cup\left\{i_{h}^{\prime \prime \prime}\right\}_{h=1}^{k \prime \prime} \cup\left\{q_{h}\right\}_{h=1}^{m}\right)} \int_{S / z^{2}}^{T / z^{2}} d u\left\langle S_{u} Q+f, S_{t_{\alpha}} Q+g_{\varepsilon(\alpha)}\right\rangle
\end{aligned}
$$




$$
\begin{aligned}
& \prod_{\alpha \in\left\{1, \cdots, n \backslash \backslash\left\langle i h_{h}^{\prime}\right\}_{h=1}^{k} \cup\left\{i_{h}^{\prime \prime \prime}\right\}_{h=1}^{\prime \prime \prime} \cup\left\{q_{h-1}\right\}_{h=1}^{m} \cup\left\{i_{h}^{\prime}-1 i_{h=1}^{k}\right)\right.} \int_{S^{\prime} / z^{2}}^{T^{\prime} / z^{2}} d u\left\langle S_{u} Q+g_{1-\varepsilon(\alpha)}, S_{u} Q+f^{\prime}\right\rangle \cdot \\
& \prod_{h=1}^{k^{\prime \prime \prime}}\left\langle Q^{-} g_{\varepsilon\left(i h^{\prime}\right)}, Q^{-} g_{1-\varepsilon\left(i^{\prime \prime \prime}\right)}\right\rangle_{c} \\
& \left\langle W\left(z \int_{S / z^{2}}^{T / z^{2}} Q+S_{u} f d u\right) \Phi_{F} \otimes W\left(z^{2} \int_{S / z^{2}}^{T / z^{2}} Q^{-} S_{u} f d u\right) \Phi_{F}^{\epsilon},\right. \\
& \left.W\left(z \int_{S^{\prime} / z^{2}}^{T^{\prime} / z^{2}} Q+S_{u} f^{\prime} d u\right) \Phi_{F} \otimes W\left(z^{2} \int_{S^{\prime} / z^{2}}^{T^{\prime} / z^{2}} Q-S_{u} f^{\prime} d u\right) \Phi_{F}^{\iota}\right\rangle
\end{aligned}
$$

where $\left\{i_{h}^{\prime}-1\right\}_{h=1}^{k} \bigcap\left\{i_{h}^{\prime}\right\}_{h=1}^{h}=\emptyset$ dues to $\left\{p_{h}^{\prime}\right\}_{h=1}^{k} \subset\{1, \ldots, n\} \backslash\left\{i_{h}^{\prime}\right\}_{h=1}^{k} ;\left\{q_{h}-\right.$ $1\}_{h=1}^{m} \cap\left\{i_{h}^{\prime}-1, i_{h}^{\prime}\right\}_{h=1}^{k}=\emptyset$ dues to $\left\{p_{h}\right\}_{h=1}^{m} \subset\{1, \ldots, n\} \backslash\left\{i_{h}^{\prime}\right\}_{h=1}^{k}$ and $\mid\left\{p_{h}\right\}_{h=1}^{m} \cup$ $\left\{p_{h}^{\prime}\right\}_{h=1}^{k} \mid=m+k$.

Remark. In (4.21), we consider the integral of the function

$$
\prod_{h=1}^{m}\left|\left\langle S_{t_{p_{t}}} Q+g_{1-\varepsilon\left(p_{h}\right),} S_{t_{o_{\alpha}}} Q+g_{\varepsilon\left(q_{h}\right)}\right\rangle\right| \cdot \prod_{h=1}^{k}\left|\left\langle S_{t p_{h}^{\prime}} Q+g_{1-\varepsilon\left(p_{h}^{\prime}\right),} S_{t_{t_{i}}} Q+g_{\varepsilon\left(i_{h}^{\prime}\right)}\right\rangle\right|
$$

but not of the function

$$
\prod_{h=1}^{m}\left|\left\langle S_{t_{p^{\prime}}} Q+g_{1-\varepsilon\left(p_{h}\right)}, S_{t_{p_{n}}} Q+g_{\varepsilon\left(q_{h}\right)}\right\rangle\right| \cdot \prod_{h=1}^{k}\left|\left\langle Q^{-} S_{t_{t_{\sigma(h)}}} g_{\varepsilon\left(t_{o(t h)}\right)}, Q^{-} S_{t_{i,}} g_{1-\varepsilon\left(i_{h}^{\prime}\right)}\right\rangle_{\iota}\right|
$$

this is due to the fact that if in (4.24) $p_{h}=q_{h}-1$ for any $h=1, \ldots, m$ but there exists a $h \in\{1, \ldots, k\}$ such that $i_{\sigma(h)}<i_{h}^{\prime}-1$, the limit

$$
\lim _{z \rightarrow 0} z^{2(n-k-m)} \int_{0}^{t / z^{2}} d t_{1} \int_{0}^{t_{1}} d t_{2} \cdots \int_{0}^{t_{n-1}} d t_{n}
$$

$$
\begin{aligned}
& \prod_{h=1}^{m}\left|\left\langle S_{t_{p h}} Q_{+} g_{1-\varepsilon\left(p_{h}\right),} S_{t_{p,}} Q+g_{\varepsilon\left(q_{h}\right)}\right\rangle \cdot\right| \prod_{h=1}^{k}\left|\left\langle S_{t_{p i}} Q+g_{1-\varepsilon\left(p_{h}^{\prime}\right)}, S_{t_{t h}} Q+g_{\varepsilon\left(p_{h}^{\prime}\right)}\right\rangle\right| \\
& \prod_{h=1}^{k}\left|\left\langle Q^{-} S_{t_{i_{\sigma o(h)}}} g_{\varepsilon\left(i_{\sigma(k)}\right)}, Q^{-} S_{t_{h}^{\prime}} g_{1-\varepsilon\left(i^{\prime}\right)}\right\rangle_{\iota}\right|
\end{aligned}
$$

may be not zero. For example, $n=4, k=1, m=2, i_{1}=1, i_{1}^{\prime}=4, q_{1}=2, q_{1}=$ 3 , then, (4.25) becomes

$$
\begin{gathered}
\lim _{z \rightarrow 0} z^{2(4-2-1)} \cdot \int_{0}^{t / z^{2}} d t_{1} \int_{0}^{t_{1}} d t_{2} \int_{0}^{t_{2}} d t_{3} \int_{0}^{t_{3}} d t_{4} \\
\left|\left\langle S_{t_{1}} Q_{+} g_{1-\varepsilon(1)}, S_{t_{2}} Q_{+} g_{\varepsilon(2)}\right\rangle\right| \cdot\left|\left\langle S_{t^{2}} Q_{+} g_{1-\varepsilon(2)}, S_{t_{3}} Q_{+} g_{\varepsilon(3)}\right\rangle\right| \cdot \\
\left|\left\langle S_{t_{3}} Q_{+} g_{1-\varepsilon(3)}, S_{t_{4}} Q_{+} g_{\varepsilon(4)}\right\rangle\right| \cdot\left|\left\langle Q^{-} S_{t_{1}} g_{\varepsilon(1)}, Q^{-} S_{t_{4}} g_{1-\varepsilon(4)}\right\rangle_{i}\right|
\end{gathered}
$$




$$
\begin{gathered}
=\lim _{z \rightarrow 0} \int_{0}^{t} d t_{1} \int_{-t_{1} / z^{2}}^{0} d t_{2}\left|\left\langle Q_{+} g_{1-\varepsilon(1)}, S_{t_{2}} Q_{+} g_{\varepsilon(2)}\right\rangle\right| \\
\int_{-t_{1} / z^{2-t_{2}}}^{0} d t_{3}\left|\left\langle Q_{+} g_{1-\varepsilon(2)}, S_{t_{3}} Q_{+} g_{\varepsilon(3)}\right\rangle\right| \\
\int_{-t_{1} / z^{2-t_{2}-t_{3}}}^{0} d t_{4}\left|\left\langle Q+Q_{1-\varepsilon(3)}, S_{t_{4}} Q_{+} g_{\varepsilon(4)}\right\rangle\right| \cdot\left|\left\langle Q^{-} g_{\varepsilon(1)}, Q^{-} S_{t_{4}+t_{3}+t_{2}} g_{1-\varepsilon(4)}\right\rangle_{\iota}\right| .
\end{gathered}
$$

We shall prove that this kind of limit exists and is, usually, not equal to zero.

Our fifth step is to prove that as $z \rightarrow 0$ only those terms can remain, in which the set of indices $\left\{i_{h}, i_{h}^{\prime}\right\}_{h=1}^{k}$ satisfies

$$
i_{1}<i_{1}^{\prime}<\cdots<i_{k}<i_{k}^{\prime}
$$

THEOREM (4.8). In the right hand side of (4.22), those terms in which (4.27) does not hold will tend to zero as $z \rightarrow 0$.

Before proving the theorem we first prove a lemma:

Lemma (4.9). Suppose that $f \in L^{1}\left(\mathbf{R}_{+}\right)$and is bounded and $m \geq 3$, then

$$
z^{-2\left(m^{\prime}+1\right)} \int_{0}^{t} d t_{1} \cdots \int_{0}^{t m-1} d t_{m} f\left(\frac{t_{m}-t_{1}}{z^{2}}\right) f\left(\frac{t_{m}-t_{m-1}}{z^{2}}\right) \cdot \prod_{h=1}^{m^{\prime}} f\left(\frac{t_{q_{h}}-t_{q_{h-1}}}{z^{2}}\right) \rightarrow 0
$$

for $m^{\prime}<m-2$, where, $2 \leq q_{1}<\cdots<q_{m^{\prime}} \leq m-1$.

Proof. If $q_{1}>2$ or $q_{m^{\prime}}<m-1$, by the boundedness of $f$, the left hand side of $(4.28)$ is less or equal to

$$
C \cdot z^{-2\left(m^{\prime}+1\right)} \int_{0}^{t} d t_{1} \cdots \int_{0}^{t m-1} d t_{m} f\left(\frac{t_{m}-t_{1}}{z^{2}}\right) \cdot \prod_{h=1}^{m^{\prime}} f\left(\frac{t_{q_{h}}-t_{q_{h}-1}}{z^{2}}\right)
$$

where $C$ is a constant. It is clear that (4.29) is a type II term in the sense of [1], therefore by the same arguments as those of the proof of Lemma (3.3) in [1] (4.28) follows.

Now, suppose that

$$
q_{1}=2 \quad q_{m^{\prime}}=m-1 .
$$

Introduce the connected decomposition

$$
\left\{q_{h}\right\}_{h=1}^{m^{\prime}}=\left\{q_{h}\right\}_{h=1}^{x_{1}} \bigcup\left\{q_{h}\right\}_{h=x_{1}+1}^{x_{2}} \cup \cdots \cup\left\{q_{h}\right\}_{h=x_{y-1}+1}^{m^{\prime}}
$$

characterized by the following properties:

(i) $q_{1}=q_{2}-1=\cdots=q_{x_{1}}-\left(x_{1}-1\right), \cdots, q_{x_{v-1}}=q_{x_{v-1}+1}-1=\cdots=q_{m^{\prime}}$ $-\left(m^{\prime}-x_{y-1}-1\right)$;

(ii) $q_{x_{1}}+1<q_{x_{1}+1}, \cdots, q_{x_{v-1}}+1<q_{x y-1+1}$. 
Then, similarly to the proof of Lemma (3.3) of [1], one has

$$
\begin{aligned}
& z^{-2\left(m^{\prime}+1\right)} \int_{0}^{t} d t_{1} \cdots \int_{0}^{t_{m-1}} d t_{m} f\left(\frac{t_{m}-t_{1}}{z^{2}}\right) f\left(\frac{t_{m}-t_{m-1}}{z^{2}}\right) \cdot \prod_{h=1}^{m^{\prime}} f\left(\frac{t_{q_{h}}-t_{q_{h-1}}}{z^{2}}\right) \\
& \leq z^{-2} \int_{0}^{t} d t_{1} \cdots \int_{0}^{t_{q_{1}-2}^{\prime}} d t_{\dot{q}_{1}-1}^{\prime} \int_{-t q_{1-1} / z^{2}}^{0} f\left(t_{q_{1}}^{\prime} d t_{q_{1}}^{\prime} \cdots\right. \\
& \int_{-t q_{1}-1 / z^{2}}^{0} f\left(t_{q_{x_{1}}}\right) d t_{q_{x_{1}}}^{\prime} \int_{0}^{t q_{1}-1} d t_{q_{x_{1}}+1}^{\prime} \\
& \cdots \int_{0}^{t_{q_{x_{1}+1^{-2}}}^{\prime}} d t_{q_{x_{1}+1}-1}^{\prime} \int_{-t_{q_{x_{1}+1^{-1}} / z^{2}}^{\prime}}^{0} f\left(t_{q_{x 1+1}}^{\prime}\right) d t_{q_{x 1+1}}^{\prime} \cdots \int_{-t_{q_{x_{1}+1^{-1}} / z^{2}}}^{0} f\left(t_{q_{x_{2}}}^{\prime}\right) d t_{q_{x_{2}}}^{\prime} \ldots \\
& \int_{0}^{t^{\prime} x_{x_{y-1}}{ }^{-2}} d t_{q_{x_{y-1}}-1}^{\prime} \int_{-t_{x_{y+1}}^{\prime}{ }^{-1 / z^{2}}}^{0} f\left(t_{q_{x_{y-1}}}^{\prime}\right) d t_{q_{x_{y-1}}}^{\prime} \cdots
\end{aligned}
$$

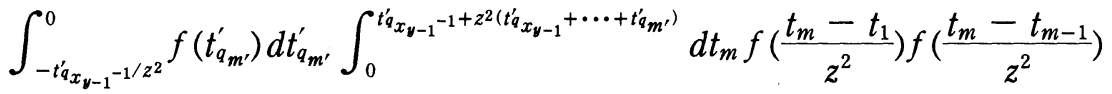

where, since $q_{m^{\prime}}=m-1$, then,

$$
t_{m-1}:=t_{a_{x_{y-1}}-1}^{\prime}+z^{2}\left(t_{a_{x_{y-1}}}^{\prime}+\cdots+t_{q_{m}}^{\prime}\right) .
$$

With the change of variable

$$
\left(t_{m}-t_{1}\right) / z^{2}=s_{m}
$$

the right hand side of (4.30) is majorized by

$$
\begin{gathered}
C_{n} \cdot \int_{0}^{t} d t_{1} \cdots \int_{0}^{t_{q_{1}-2}^{\prime}} d t_{q_{1}-1}^{\prime} \int_{0}^{t_{q_{1}-1}^{\prime}} d t_{q_{1}+1}^{\prime} \cdots \\
\cdots \int_{0}^{t^{\prime} x_{x_{y-1}-2}} d t_{q_{x_{y-1}-1}^{\prime}}^{\prime} \cdots \int_{-t_{1} / z^{2}}^{-\left(t_{1}-t^{\prime} x_{\left.x_{y-1}-1\right) / z^{2}+t_{q_{x-1}}^{\prime}}+\cdots+t^{\prime} q_{m}^{\prime}\right.} d s_{m} f\left(s_{m}\right)
\end{gathered}
$$

and this vanishes as $z \rightarrow 0$.

Proof of Theorem (4.8). It is enough to show that if either there exists a $h=1, \ldots, k-1$, such that

$$
\left|\left\{i_{h}^{\prime}\right\}_{h=1}^{k} \cap\left\{i_{h}+1, \cdots, i_{h+1}-1\right\}\right| \neq 1
$$

or there exists a $h=1, \ldots, k-1$, such,that

$$
\left|\left\{i_{h}\right\}_{h=1}^{k} \cap\left\{i_{h}^{\prime}+1, \cdots, i_{h+1}^{\prime}-1\right\}\right| \neq 1
$$

then,

$$
\lim _{z \rightarrow 0} z^{2(n-k-m)} \cdot \int_{0}^{t / z^{2}} d t_{1} \int_{0}^{t_{1}} d t_{2} \cdots \int_{0}^{t_{n-1}} d t_{n} \prod_{h=1}^{m}\left|\left\langle S_{t_{q-1}} Q+g_{1-\varepsilon\left(q_{k}-1\right)}, S_{t_{o x}} Q+g_{\varepsilon\left(q_{h}\right)}\right\rangle\right|
$$


$\prod_{h=1}^{k}\left|\left\langle S_{t_{i_{h}-1}} Q+g_{1-\varepsilon\left(i_{h}^{\prime}-1\right)}, S_{t_{i_{i}}} Q+g_{\varepsilon\left(i_{h}^{\prime}\right)}\right\rangle\right| \prod_{h=1}^{k}\left|\left\langle Q^{-} S_{t_{t_{h}}} g_{\varepsilon\left(t_{h}\right)}, Q^{-} S_{t_{t_{h}}} g_{1-\varepsilon\left(i_{h}^{\prime}\right)}\right\rangle\right|=0$

We shall prove (4.35) separately distinguishing 4 cases.

(i) There exists a $h=1, \ldots, k-1$, such that

$$
\left|\left\{i_{h}^{\prime}\right\}_{h=1}^{k} \cap\left\{i_{h}+1, \cdots, i_{h+1}-1\right\}\right|=0 .
$$

In this case, denoting by $h_{0}$ the minimum $h$ satisfying (4.36), since $i_{h}<i_{h}^{\prime}$ and $i_{h}^{\prime} \notin\left\{i_{h}\right\}_{h=1}^{k}$ for any $h$, we have

$$
i_{h_{0-1}}^{\prime}<i_{h_{0}}<i_{h_{0+1}}<i_{h_{0}}^{\prime}
$$

and therefore

$$
\begin{aligned}
& \prod_{h=1}^{m}\left|\left\langle S_{t_{q^{\prime}-1}} Q+g_{1-\varepsilon\left(q_{h}-1\right)}, S_{t_{t_{k}}} Q+g_{\varepsilon\left(q_{h}\right)}\right\rangle\right\rangle \mid \text {. }
\end{aligned}
$$

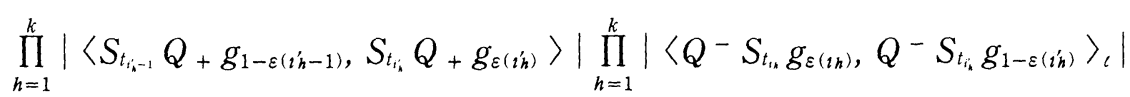

$$
\begin{aligned}
& \leq C_{n} \cdot z^{2(n-k-m)} \cdot \int_{0}^{t / z^{2}} d t_{1} \int_{0}^{t_{1}} d t_{2} \cdots \int_{0}^{t_{n-1}} d t_{n} \\
& \prod_{h=1}^{m}\left|\left\langle S_{t_{q \alpha-1}} Q+g_{1-\varepsilon\left(q_{h}-1\right)} S_{t_{q_{*}}} Q+g_{\varepsilon\left(q_{h}\right)}\right\rangle\right| \cdot \\
& \prod_{h \in\left\{1, \cdots, \hat{n}_{0}, \cdots, k\right\}}\left|\left\langle S_{t_{i-1}} Q+g_{1-\varepsilon\left(i^{\prime}-1\right)}, S_{t_{i,}} Q+g_{\varepsilon\left(i_{h}^{\prime}\right)}\right\rangle\right| \\
& \left|\left\langle Q^{-} S_{t_{i_{0}}} g_{\varepsilon\left(t_{h_{0}}\right)}, Q^{-} S_{t_{i_{0}}} g_{1-\varepsilon\left(t_{h_{0}}\right)}\right\rangle_{\iota}\right|=: J_{n}(1, t)
\end{aligned}
$$$$
z^{2(n-k-m)} \cdot \int_{0}^{t^{\prime} z^{2}} d t_{1} \int_{0}^{t_{1}} d t_{2} \cdots \int_{0}^{t_{n-1}} d t_{n}
$$

where, $C_{n}$ is a constant. Now, we shall show that $J_{n}(1, t)$ goes to zero as $z \rightarrow 0$. In the following, we denote $\left\{i_{h}^{\prime}\right\}_{h=1}^{k} \cup\left\{q_{h}\right\}_{h=1}^{m}$ as $\left\{\bar{q}_{h}\right\}_{h=1}^{m+k}$. By (4.36), one knows that

$$
\left\{i_{h}^{\prime}, i_{h}^{\prime}-1\right\}_{h \in\left\{1, \cdots, \hat{h}_{0}, \cdots, k\right\}} \cap\left\{i_{h_{0}}+1, \cdots, i_{h_{0}+1}-1\right\}=\emptyset .
$$

Moreover, with the change of variable $(4.10 \mathrm{a})$ and enlarging $t_{h_{0}-1}$ to $t$, one finds that

$$
\begin{aligned}
& j_{n}(1, t)=C_{n} \cdot z^{-2(k+m)} \cdot \int_{0}^{t} d t_{1} \int_{0}^{t_{1}} d t_{2} \cdots \int_{0}^{t_{n-1}} d t_{n} \\
& \prod_{h=1}^{m}\left|\left\langle Q_{+} g_{1-\varepsilon\left(q_{h}-1\right)}, \frac{S_{t_{q_{h}}-t_{q-1}}}{z^{2}} Q_{+} g_{\varepsilon\left(q_{h}\right)}\right\rangle\right| \cdot \\
& \prod_{h \in\left\{1, \cdots, \hat{h_{0}}, \cdots, k\right\}}\left|\left\langle Q+g_{1-\varepsilon\left(i_{h}^{\prime}-1\right)}, \frac{S_{\frac{t_{i_{i}}-t_{i j-1}}{z^{2}}}}{z^{2}} Q_{+} g_{\varepsilon\left(i_{h}^{\prime}\right)}\right\rangle\right|
\end{aligned}
$$




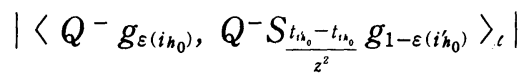

$$
\begin{aligned}
& \leq C_{n} \cdot z^{-2(k+m)} \cdot \int_{0}^{t} d t_{1} \cdots \int_{0}^{t_{h_{0}-2}} d t_{h_{0-1}} \cdot \int_{0}^{t} d t_{h 0} \cdots \int_{0}^{t n-1} d t_{n} \\
& \prod_{h=1}^{m}\left|\left\langle Q+g_{1-\varepsilon\left(q_{h}-1\right)}, S_{\frac{t_{q_{4}}-t_{q_{h}}-1}{z^{2}}} Q+g_{\varepsilon\left(q_{h}\right)}\right\rangle\right| \text {. } \\
& \prod_{h=1, \cdots, \hat{n}_{0}, \cdots, k}\left|\left\langle Q+g_{1-\varepsilon\left(i_{h}^{\prime}-1\right)}, \frac{S_{t_{i j}-t_{i}-1}}{z^{2}} Q+g_{\varepsilon\left(i_{h}^{\prime}\right)}\right\rangle\right| \\
& \left|\left\langle Q^{-} g_{\varepsilon\left(i_{h_{0}}\right)}, Q^{-} S_{\frac{t_{i_{0}}-t_{\text {ino }}}{z^{2}}} g_{1-\varepsilon\left(i_{h_{0}}\right)}\right\rangle_{l}\right| \text {. }
\end{aligned}
$$

Putting

$$
p:=\left|\left\{h \in\{1, \ldots, m\}: i_{h_{0}}<q_{h}<i_{h_{0}}^{\prime}\right\}\right|
$$

and

$$
\left\{\beta_{h}\right\}_{h=1}^{h}:=\left\{q_{h}: i_{h_{0}}<q_{h}<i_{h 0}^{\prime}, h=1, \ldots, m\right\}
$$

Then,

$$
\begin{aligned}
& \prod_{h=1}^{p}\left|\left\langle Q+g_{1-\varepsilon\left(\beta_{h}-1\right)}, S_{\frac{t_{\beta_{h}}-t_{\beta_{1}}-1}{z^{2}}} Q+g_{\varepsilon\left(\beta_{h}\right)}\right\rangle\right| \text {. }
\end{aligned}
$$

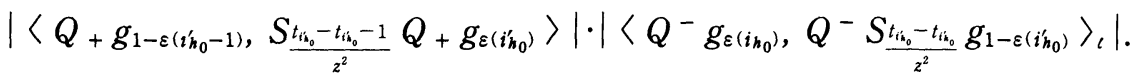

Applying Lemma (4.8) to the right hand side of (4.43), we know that term goes to zero as $z \rightarrow 0$.

(ii) There exists a $h=1, \ldots, k-1$, such that

$$
\left|\left\{i_{h}^{\prime}\right\}_{h=1}^{k} \cap\left\{i_{h}+1, \cdots, i_{h+1}-1\right\}\right|=d \geq 2 .
$$

In this case, denoting by $h_{0}$ the minimum $h$ satisfying (4.44), then since $i_{h}$ $<i_{h}^{\prime}$ for any $h=1, \ldots, k$, we know that not only $i_{h_{0}}^{\prime} \in\left\{i_{h_{0}}+1, \cdots, i_{h_{0}+1}-1\right\}$ but also $i_{h_{0}-1}^{\prime} \in\left\{i_{h_{0}}+1, \cdots, i_{h_{0}+1}-1\right\}$, i.e.

$$
i_{h 0-1}<i_{h 0}<i_{h 0-1}^{\prime}<i_{h 0}^{\prime} .
$$

Considering the piece $i_{h_{0}-1}^{\prime}, \cdots, i_{h_{0}}^{\prime}$, one gets (4.43) again. This implies (4.35).

(iii) There exists a $h=1, \ldots, k-1$, such that

$$
\left|\left\{i_{h}\right\}_{h=1}^{k} \cap\left\{i_{h}^{\prime}+1, \cdots, i_{h+1}^{\prime}-1\right\}\right|=0 .
$$

In this case, $i_{h+1}<i_{h}^{\prime}$, considering the piece $i_{h}, \cdots, i_{h}^{\prime}$, one gets (4.43) again.

(iv) There exists a $h=1, \ldots, k-1$, such that 


$$
\left|\left\{i_{h}\right\}_{h=1}^{k} \cap\left\{i_{h}^{\prime}+1, \cdots, i_{h+1}^{\prime}-1\right\}\right| \geq 2 .
$$

In this case, since $i_{h}<i_{h}^{\prime}, h=1, \ldots, k$, we can write (4.40) as

$$
\left|\left\{i_{j}\right\}_{j=h+1}^{k} \cap\left\{i_{h}^{\prime}+1, \cdots, i_{h+1}^{\prime}-1\right\}\right| \geq 2
$$

therefore there exist $h_{1}, h_{2} \in\{h+1, \ldots, k\}$ such that

$$
i_{h}^{\prime}<i_{h_{1}}<i_{h_{2}}<i_{h+1}^{\prime}
$$

considering the piece $i_{h_{1}}, \cdots, i_{h_{1}+1}^{\prime}$, one gets (4.43) again.

From the above arguments our proof follows.

Summing up, we know that as $z \rightarrow 0$ the limit of $I_{n}^{\varepsilon}$ is equal to the limit of the following quantity:

$$
\begin{aligned}
& \mathrm{I}_{n}^{\varepsilon}(4, z, t):=\sum_{k=0}^{[n / 2]} \sum_{1 \leq i_{1}<i_{1}^{\prime}<\cdots<i k<i_{k}^{\prime} \leq n} \\
& \sum_{k^{\prime \prime \prime}=0}^{n-2 k} \sum_{1 \leq i_{1}^{\prime \prime \prime}<\cdots<i_{k^{\prime \prime \prime \prime}} \leq n} \\
& \left\{i_{h}^{\prime \prime \prime}\right\}_{h=1}^{\prime \prime \prime} \cap\left(\{i h\}_{h=1}^{k} \cup\left\{i_{h}^{\prime}\right\}_{h=1}^{k}\right)=\varnothing \\
& \sum_{m=0}^{n-2 k-k^{\prime \prime \prime}} \\
& \sum_{1 \leq q_{1}<\cdots<q_{m} \leq n} \\
& \left\{q_{h}\right\}_{h=1}^{m} \subset\{1, \cdots, n\} \backslash\left(\left\{i_{h}, i_{h}^{\prime}\right\}_{h=1}^{k} \cup\left\{i_{h}^{\prime \prime \prime}\right)_{h=1}^{\prime \prime \prime \prime}\right) ;\left\{q_{h}-1\right\}_{h=1}^{m} \cap\left(\left\{i i_{n} i_{h}^{\prime}-1\right\}_{h=1}^{k} \cup\left\{i_{h}^{\prime \prime \prime}\right\}_{h=1}^{\prime \prime \prime}\right)=\varnothing \\
& z^{2(n-k-m)} \cdot \int_{0}^{t / z^{2}} d t_{1} \int_{0}^{t_{1}} d t_{2} \cdots \int_{0}^{t_{n-1}} d t_{n} \\
& \prod_{h=1}^{k} \mid\left\langle Q^{-} S_{t_{1,}} g_{\varepsilon\left(i_{h}\right)}, Q^{-} S_{t_{i,}} g_{1-\varepsilon\left(i_{h}^{\prime}\right)}\right\rangle_{\iota} \\
& \prod_{h=1}^{m} \mid\left\langle S_{t_{q-1}} Q+g_{1-\varepsilon\left(q_{h}-1\right)}, S_{t_{q_{\alpha}}} Q+g_{\varepsilon\left(q_{h}\right)}\right\rangle \cdot \prod_{h=1}^{k}\left\langle S_{t_{\mathrm{i}-1}} Q+g_{1-\varepsilon\left(i^{\prime}-1\right)}, S_{t_{\mathrm{i}}} Q+g_{\varepsilon\left(i_{h}^{\prime}\right)}\right\rangle \\
& \prod_{\alpha \in\left\{1, \cdots, n \nmid \backslash\left(\left\{h, i_{h}^{\prime}\right\}_{h=1}^{k} \cup\left\{t_{h}^{\prime \prime \prime}\right\}_{h=1}^{k^{\prime \prime \prime} \cup} \cup\left\{q_{h}\right\}_{h=1}^{m}\right.\right.} \int_{S / z^{2}}^{T / z^{2}} d u\left\langle S_{u} Q+f, S_{t_{\alpha}} Q+g_{\varepsilon(\alpha)}\right\rangle \\
& \prod_{\alpha \in\{1, \cdots, n\} \backslash\left\{i_{h}^{\prime}\right\}_{h=1}^{k} \cup\left\{i_{h}^{\prime \prime \prime}\right\}_{h=1}^{k^{\prime \prime \prime}} \cup\left\{q_{h-1}\right\}_{h=1}^{m} \cup\left\{i_{h-1}^{\prime}\right\}_{h=1}^{k}} \int_{S^{\prime} / z^{2}}^{T^{\prime} / 2} d u\left\langle S_{t_{\alpha}} Q+g_{1-\varepsilon(\alpha)}, S_{u} Q+f^{\prime}\right\rangle . \\
& \prod_{h=1}^{k^{\prime \prime \prime \prime}}\left\langle Q^{-} g_{\varepsilon\left(i h^{\prime \prime}\right)}, Q^{-} g_{1-\varepsilon\left(i h^{\prime \prime}\right)}\right\rangle_{\iota} \\
& \left\langle W\left(z \int_{S / z^{2}}^{T / z^{2}} Q+S_{u} f d u\right) \Phi_{F} \otimes W\left(z^{2} \int_{S / z^{2}}^{T / z^{2}} Q-S_{u} f d u\right) \Phi_{F}^{\iota},\right. \\
& \left.W\left(z \int_{S^{\prime} / z^{2}}^{T^{\prime} / z^{2}} Q+S_{u} f^{\prime} d u\right) \Phi_{F} \otimes W\left(z^{2} \int_{S^{\prime} / z^{2}}^{T^{\prime} / z^{2}} Q^{-} S_{u} f^{\prime} d u\right) \Phi_{F}^{\epsilon}\right\rangle .
\end{aligned}
$$


Now let us investigate the limit. In order to do this we shall first prove two Lemmata.

Lemma (4.10). For each $m \in \mathbf{N},\left\{f_{h}\right\}_{h=1}^{m+1} \subset L^{1}(\mathbf{R})$ and bounded, the limit

$$
\lim _{z \rightarrow 0} z^{-2 m} \int_{0}^{t} d t_{1} \cdots \int_{0}^{t m} d t_{m+1} f_{1}\left(\frac{t_{m+1}-t_{1}}{z^{2}}\right) f_{m+1}\left(\frac{t_{m+1}-t_{m}}{z^{2}}\right) \cdot \prod_{h=1}^{m-1} f_{h+1}\left(\frac{t_{h+1}-t_{h}}{z^{2}}\right)
$$

exists.

Proof. With the change of variables

$$
\left(t_{h+1}-t_{h}\right) / z^{2} \hookrightarrow t_{h+1}, h=1, \ldots, m
$$

we have that

$$
\begin{gathered}
z^{-2 m} \int_{0}^{t} d t_{1} \cdots \int_{0}^{t_{m}} d t_{m+1} f_{1}\left(\frac{t_{m+1}-t_{1}}{z^{2}}\right) f_{m+1}\left(\frac{t_{m+1}-t_{m}}{z^{2}}\right) \cdot \prod_{h-1}^{m-1} f_{h+1}\left(\frac{t_{h+1}-t_{h}}{z^{2}}\right) \\
=\int_{0}^{t} d t_{1} \cdots \int_{-t_{1} / z^{2}}^{0} d t_{2} f_{2}\left(t_{2}\right) \int_{-t_{1} / z^{2}-t_{2}}^{0} d t_{3} f_{3}\left(t_{3}\right) \cdots \\
\int_{-t / z^{2}-t^{2}-\cdots-t m}^{0} d t_{m+1} f_{1}\left(t_{m+1}+\cdots+t_{2}\right) f_{m+1}\left(t_{m+1}\right) \\
=: M(m, z, t) .
\end{gathered}
$$

Moreover,

$$
\begin{gathered}
\leq \mid \int_{0}^{t} d t_{1} \cdots \int_{-t_{1} / z_{1}^{2}}^{0} d t_{2} f_{2}\left(t_{2}\right) \int_{-t_{1} / z_{1}^{2}-t_{2}}^{0} d t_{3} f_{3}\left(t_{3}\right) \cdots \\
\int_{-t_{1} / z_{1}^{2}-t_{2}-\cdots-t m}^{0} d t_{m+1} f_{1}\left(t_{m+1}+\cdots+t_{2}\right) f_{m+1}\left(t_{m+1}\right) \\
-\int_{0}^{t} d t_{1} \cdots \int_{-t_{1} / z_{1}^{2}}^{0} d t_{2} f_{2}\left(t_{2}\right) \int_{-t_{1} / z_{1}^{2}-t_{2}}^{0} d t_{3} f_{3}\left(t_{3}\right) \cdots \\
\quad \int_{-t_{1} / z_{2}^{2}-t_{2}-\cdots-t_{m}}^{0} d t_{m+1} f_{1}\left(t_{m+1}+\cdots+t_{2}\right) f_{m+1}\left(t_{m+1}\right) \\
+\int_{0}^{t} d t_{1}\left|\int_{-t_{1} / z_{2}^{2}}^{-t_{1} / z_{1}^{2}} d t_{2}\right| f_{2}\left(t_{2}\right)|| \cdot\left(\max _{1 \leq h \leq m+1} \int_{-\infty}^{0}\left|f_{h}\right| d t\right)^{m-1} \sup _{t \in \mathbf{R}}|f(t)| \\
\leq \cdots \leq m \cdot \int_{0}^{t} d t_{1}\left|\int_{-t_{1} / z_{2}^{2}}^{-t_{1} / z_{1}^{2}} d t_{2}\right| f_{2}\left(t_{2}\right)|| \cdot\left(\max _{1 \leq h \leq m+1} \int_{-\infty}^{0}\left|f_{h}\right| d t\right)^{m-1} \sup _{t \in \mathbf{R}}|f(t)|
\end{gathered}
$$$$
\longrightarrow 0
$$ 
as $z_{1}, z_{2} \rightarrow 0$. Therefore by the Cauchy principle the proof is finished.

LEMMA (4.11). With the above symbols and assumptions

$$
\begin{gathered}
\lim _{z \rightarrow 0} M(m, z, t)=\int_{0}^{t} d t_{1} \int_{-\infty}^{0} d t_{2} f_{2}\left(t_{2}\right) \cdots \int_{-\infty}^{0} d t_{m} f_{m}\left(t_{m}\right) \\
\int_{-\infty}^{0} d t_{m+1} f_{m+1}\left(t_{m+1}\right) f_{1}\left(t_{m+1}+\cdots+t_{2}\right)
\end{gathered}
$$

Proof.

$$
\begin{aligned}
& \left|M(m, z, t)-\int_{0}^{t} d t_{1} \int_{-\infty}^{0} d t_{2} f_{2}\left(t_{2}\right) \cdots \int_{-\infty}^{0} d t_{m+1} f_{m+1}\left(t_{m+1}\right) f_{1}\left(t_{m+1}+\cdots+t_{2}\right)\right| \\
& \leq \mid \int_{0}^{t} d t_{1} \cdots \int_{-T}^{0} d t_{2} f_{2}\left(t_{2}\right) \int_{-t_{1} / z_{1}^{2}-t_{2}}^{0} d t_{3} f_{3}\left(t_{3}\right) \cdots \\
& \int_{-t_{1} / z^{2}-t_{2}-\cdots-t_{m}}^{0} d t_{m+1} f_{1}\left(t_{m+1}+\cdots+t_{2}\right) f_{m+1}\left(t_{m+1}\right) \\
& -\int_{0}^{t} d t_{1} \int_{-\infty}^{0} d t_{2} f_{2}\left(t_{2}\right) \cdots \int_{-\infty}^{0} d t_{m+1} f_{m+1}\left(t_{m+1}\right) f_{1}\left(t_{m+1}+\cdots+t_{2}\right) \\
& +C_{m}\left|\int_{0}^{t} d t_{1} \int_{-T}^{-t_{1} / z^{2}}\right| f_{2}\left(t_{2}\right)\left|d t_{2}\right| \\
& \leq \mid \int_{0}^{t} d t_{1} \int_{-T}^{0} d t_{2} f_{2}\left(t_{2}\right) \int_{-T}^{0} d t_{3} f_{3}\left(t_{3}\right) \int_{-t_{1} / z_{1}^{2}-t_{2}-t_{3}}^{0} d t_{4} f_{4}\left(t_{4}\right) \cdots \\
& \int_{-t_{1} / z_{1}^{2}-t_{2}-\cdots-t_{m}}^{0} d t_{m+1} f_{1}\left(t_{m+1}+\cdots+t_{2}\right) f_{m+1}\left(t_{m+1}\right) \\
& -\int_{0}^{t} d t_{1} \int_{-\infty}^{0} d t_{2} f_{2}\left(t_{2}\right) \cdots \int_{-\infty}^{0} d t_{m+1} f_{m+1}\left(t_{m+1}\right) f_{1}\left(t_{m+1}+\cdots+t_{2}\right) \\
& +C_{m}\left|\int_{0}^{t} d t_{1} \int_{-T}^{0}\right| f_{2}\left(t_{2}\right)\left|d t_{2} \int_{-T}^{-t_{1} / z^{2}-t_{2}}\right| f_{3}\left(t_{3}\right)\left|d t_{3}\right| \\
& +C_{m}\left|\int_{0}^{t} d t_{1} \int_{-T}^{-t_{1} / z^{2}}\right| f_{2}\left(t_{2}\right)\left|d t_{2}\right| \\
& \leq \cdots \leq \cdots \\
& \leq C_{m}\left|\int_{0}^{t} d t_{1} \int_{-T}^{-t_{1} / z^{2}}\right| f_{2}\left(t_{2}\right)\left|d t_{2}\right| \\
& +C_{m}\left|\int_{0}^{t} d t_{1} \int_{-T}^{0}\right| f_{2}\left(t_{2}\right)\left|d t_{2} \int_{-T}^{-t_{1} / z^{2}-t_{2}}\right| f_{3}\left(t_{3}\right)\left|d t_{3}\right| \\
& +\cdots+ \\
& +C_{m}\left|\int_{0}^{t} d t_{1} \int_{-T}^{0}\right| f_{2}\left(t_{2}\right)\left|d t_{2} \int_{-T}^{0}\right| f_{3}\left(t_{3}\right) \mid d t_{3} \cdots
\end{aligned}
$$




$$
\begin{gathered}
\int_{-T}^{-t_{1} / z^{2}-t_{2}-\cdots-t_{m}}\left|f_{m+1}\left(t_{m+1}\right)\right| d t_{m+1} \mid \\
+\mid \int_{0}^{t} d t_{1} \int_{-T}^{0} d t_{2} f_{2}\left(t_{2}\right) \cdots \int_{-T}^{0} d t_{m+1} f_{m+1(t m+1)} f_{1}\left(t_{m+1}+\cdots+t_{2}\right)- \\
-\left|\int_{0}^{t} d t_{1} \int_{-\infty}^{0} d t_{2} f_{2}\left(t_{2}\right) \cdots \int_{-\infty}^{0} d t_{m+1} f_{m+1}\left(t_{m+1}\right) f_{1}\left(t_{m+1}+\cdots+t_{2}\right)\right|
\end{gathered}
$$

where,

$$
C_{m}:=\left(\max _{h=1, \cdots, m+1} \int_{-\infty}^{0}\left|f_{h}(t) d t\right| \bigvee \max _{h=1, \cdots, m+1} \sup _{t \in \mathbf{R}}\left|f_{h}(t)\right| \bigvee 1\right)^{m} .
$$

For each $\eta>0$, we take $T$ such that

$$
\max _{h=1, \cdots, m} \int_{-\infty}^{-T}\left|f_{h}(t)\right| d t<\eta
$$

then,

$$
\begin{gathered}
\lim _{z \rightarrow 0}\left|M(m, z, t)-\int_{0}^{t} d t_{1} \int_{-\infty}^{0} d t_{2} f_{2}\left(t_{2}\right) \cdots \int_{-\infty}^{0} d t_{m+1} f_{m+1}\left(t_{m+1}\right) f_{1}\left(t_{m+1}+\cdots+t_{2}\right)\right| \\
\leq \eta \cdot t\left(C_{m}+C_{m}^{2}+\cdots+C_{m}^{m}\right)
\end{gathered}
$$

this shows the validity of (4.55).

Using the two Lemmata, we can make our sixth step:

THEOREM (4.12).

$$
\begin{aligned}
& \lim _{z \rightarrow 0} \mathbb{I}_{n}^{\varepsilon}=\sum_{k=0}^{[n / 2]} \sum_{1 \leq i i_{1}<i_{1}^{\prime}<\cdots<i_{k}<i_{k}^{\prime} \leq n} \sum_{k^{\prime \prime \prime}=0}^{n-2 k} \sum_{1 \leq i_{1}^{\prime \prime}<\cdots<i_{k}^{\prime \prime \prime \prime} \leq n} \\
& \left\langle i i_{h}^{\prime \prime \prime}\right\}_{h=1}^{\prime \prime \prime} \cap\left(\left\langlei h h_{h=1}^{k} \cup\left\langle i_{h}^{\prime} h_{h=1}^{k}\right)=\emptyset\right.\right. \\
& \sum_{m=0}^{n-2 k-k^{\prime \prime \prime}} \sum_{1 \leq q_{1}<\cdots<q_{m} \leq n} \\
& \left\{q_{h}\right\}_{h=1}^{m} \subset\{1, \cdots, n\} \backslash\left(\left\{t_{h}, i_{h}^{\prime}\right\}_{h=1}^{k} \cup\left\langle i_{h}^{\prime \prime \prime}\right\}_{h=1}^{\prime \prime \prime}\right) ;\left\{q_{h}-1\right\}_{h=1}^{m} \cap\left(\left\langle i_{h}^{\prime}, i_{h}^{\prime}-1\right\}_{h=1}^{k} \cup\left\langle i_{h}^{\prime \prime \prime}\right\}_{h=1}^{k^{\prime \prime \prime}}\right)=\varnothing
\end{aligned}
$$

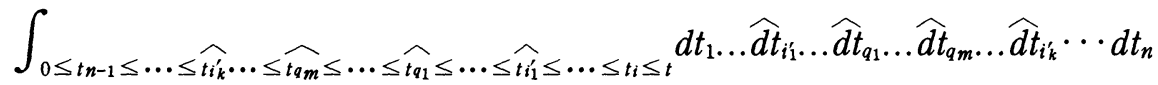

$$
\begin{aligned}
& \prod_{h=1}^{k} M\left(\varepsilon, i_{h}, i_{h}^{\prime},\left\{q_{h}\right\}_{h=1}^{m}\right) \cdot N\left(\varepsilon,\left\{i_{h+1}, i_{h}^{\prime}\right\}_{h=0}^{k},\left\{q_{h}\right\}_{h=1}^{m}\right) \\
& \prod_{h=1}^{k^{\prime \prime \prime}}\left\langle g_{1-\varepsilon\left(i^{\prime \prime}\right)}, e^{-\frac{1}{2} \beta H} g_{\varepsilon\left(i^{\prime \prime}\right)}\right\rangle \cdot\left\langle W\left(\chi_{|S, T|} \otimes f\right) \Psi, W\left(\chi_{\left|S^{\prime}, T^{\prime}\right|} \otimes f^{\prime}\right) \Psi\right\rangle
\end{aligned}
$$

with

$$
M\left(\varepsilon, i_{h}, i_{h}^{\prime},\left\{q_{h}\right\}_{h=1}^{m}\right):=\int_{-\infty}^{0} d t_{i_{h}+1} \cdots \int_{-\infty}^{0} d t_{i_{h}^{\prime}}
$$




$$
\prod_{\alpha=0}^{i_{h}-i_{h}-1}\left\langle g_{1-\varepsilon\left(i_{h}+\alpha\right),} S_{t_{w+\alpha+1}} g_{\varepsilon\left(i_{h}+\alpha+1\right)}\right\rangle \overline{\left\langle g_{\varepsilon\left(i_{h}\right)}, S_{t_{h}+\cdots+t_{n+1}} e^{-\frac{1}{2} \beta H} g_{1-\varepsilon\left(i_{h}^{\prime}\right)}\right\rangle} .
$$

Moreover, denoting

$$
\begin{gathered}
\left\{\beta_{h}\right\}_{h=1}^{\bar{m}}:=\left\{q_{h}: q_{h} \notin \bigcup_{h=1}^{k}\left\{i_{n}+1, \cdots, i_{h}^{\prime}-1\right\}, h=1, \ldots, m\right\} \\
\bar{m}=m-\sum_{h=1}^{k}\left(i_{h}^{\prime}-i_{h}-1\right)
\end{gathered}
$$

then $\left\{\beta_{h}\right\}_{h=1}^{\bar{m}}$ has the connected decomposition

$$
\left\{\beta_{h}\right\}_{h=1}^{\bar{m}}=\left\{\beta_{h}\right\}_{h=1}^{r_{1}} \cup\left\{\beta_{h}\right\}_{h=r_{1+1}}^{r_{2}} \cup \cdots \cup\left\{\beta_{h}\right\}_{h=r x-1+1}^{r_{x}^{x}}
$$

the quantity $N\left\{\varepsilon,\left\{i_{h+1}, i_{h}^{\prime}\right\}_{h=0}^{k},\left\{q_{h}\right\}_{h=1}^{m}\right)$ is defined by the following expression:

$$
\begin{aligned}
& \prod_{a \in\{1, \cdots, n\} \backslash\left\langle\left\{i_{h}^{\prime}, i_{h}\right\}_{h=1}^{k} \cup\left\{i_{h}^{\prime \prime \prime}\right\}_{h=1}^{\prime \prime \prime \prime} \cup \cup\left(q_{h}\right\}_{h=1}^{\prime}\right)}\left(f \mid g_{\varepsilon(\alpha)}\right) \cdot \chi_{|S, T|}\left(t_{\alpha}\right) \\
& \prod_{a \in\left(\left\{1, \cdots, n \nmid \backslash\left(\left\{i_{h}^{\prime}, i_{h}^{\prime}-1\right\}_{h=1}^{k} \cup\left\{i_{h}^{\prime \prime \prime}\right\}_{h=1}^{k^{\prime \prime \prime}} \cup\left\{q_{h}-1\right\}_{h=1}^{m}\right)\right) \cap\left\{1, \cdots, \beta_{1}-2\right\}\right.}\left(g_{1-\varepsilon(\alpha)} \mid f^{\prime}\right) \cdot \chi_{\left|S^{\prime}, T^{\prime}\right|}\left(t_{\alpha}\right) \\
& \cdot \chi_{\left|S^{\prime}, T^{\prime}\right|}\left(t_{\beta_{1}-1}\right)\left(g_{1-\varepsilon\left(\beta r_{1}\right)} \mid f^{\prime}\right) \\
& \prod_{a \in\left(\{1, \cdots, n\} \backslash\left(\left\{i_{h}^{\prime}, i_{h}^{\prime}-1\right\}_{h=1}^{k} \cup\left\{i_{h}^{\prime \prime \prime}\right\}_{h=1}^{k^{\prime \prime \prime}} \cup\left\{q_{h}-1\right\}_{h=1}^{m}\right)\right) \cap\left\{\beta r_{1}+1, \cdots, \beta r_{1}+1-2\right\}}\left(g_{1-\varepsilon(\alpha)} \mid f^{\prime}\right) \cdot \chi_{\left|S^{\prime}, T^{\prime}\right|}\left(t_{\alpha}\right) \\
& \text {...... } \\
& \cdot \chi_{\left\{S^{\prime}, T^{\prime}\right\}}\left(t_{\beta r_{x-1}+1-1}\right)\left(g_{1-\varepsilon\left(\beta r_{x}\right)} \mid f^{\prime}\right) \\
& \prod_{a \in\left(\{1, \cdots, n\} \backslash\left(\left\{i_{h}^{\prime}, i_{h}^{\prime}-1\right\}_{h=1}^{k} \cup\left\{q_{h}-1\right\}_{h=1}^{m}\right)\right) \cap\left\langle\beta r_{x+1}+1, \cdots, n\right\}}\left(g_{1-\varepsilon(\alpha)} \mid f^{\prime}\right) \cdot \chi_{\left|S^{\prime}, T^{\prime}\right|}\left(t_{\alpha}\right) .
\end{aligned}
$$

Proof. Using Lemma (4.11), the proof of the lemma (3.4) in [1] and dominated convergence, it is enough to show for each subset $R_{z}$ of $\mathbf{R}$, and $\xi, \eta \in K$,

$$
\lim _{z \rightarrow 0} \int_{R z}\left\langle Q+\xi, S_{t} Q+\eta\right\rangle d t=\lim _{z \rightarrow 0} \int_{R z}\left\langle\xi, S_{t} \eta\right\rangle d t .
$$

In fact

$$
\begin{aligned}
& \left|\int_{R z}\left\langle Q+\xi, S_{t} Q+\eta\right\rangle d t-\int_{R z}\left\langle\xi, S_{t} \eta\right\rangle d t\right| \\
& \quad \leq \int_{R z}\left|\left\langle Q+\xi, S_{t} Q+\eta\right\rangle-\left\langle\xi, S_{t} \eta\right\rangle\right| d t
\end{aligned}
$$

and

So,

$$
Q_{+}^{2}=\left(1-z^{2} e^{-\frac{1}{2} \beta H}\right)^{-1}=\sum_{n=0}^{\infty} z^{2 n} e^{-\frac{1}{2} \beta n H} .
$$

$$
\int_{R z}\left|\left\langle Q_{+} \xi, S_{t} Q_{+} \eta\right\rangle-\left\langle\xi, S_{t} \eta\right\rangle\right| d t \leq z^{2} \sum_{n=1}^{\infty} z^{2(n-1)}
$$




$$
\times \int_{-\infty}^{\infty}\left|\left\langle\xi, S_{t} e^{-\frac{1}{2} \beta n H} \eta\right\rangle\right| d t=z^{2} \cdot O(1) \longrightarrow 0
$$

\section{§5. The uniform estimate}

Having investigated the low density limit term by term, the present section will be devoted to study the uniform estimate.

Applying (3.24), (3.25) to (3.22), it will be obtained that

$$
\begin{aligned}
& \mid<W\left(z \int_{S / z^{2}}^{T / z^{2}} Q+S_{u} f d u\right) \Phi_{F} \otimes W\left(z^{2} \int_{S / z^{2}}^{T / z^{2}} Q^{-} S_{u} f d u\right) \Phi_{F}^{\iota}, \\
& \int_{0}^{t_{/ 2}^{2}} d t_{1} \int_{0}^{t_{1}} d t_{2} \cdots \int_{0}^{t_{n-1}} d t_{n}\left(\mathrm{I}_{n}^{\varepsilon}+\mathrm{II}_{n}^{\varepsilon}\right) \\
& \left.\cdot W\left(z \int_{S^{\prime} / z^{2}}^{T^{\prime} / z^{2}} Q+S_{u} f^{\prime} d u\right) \Phi_{F} \otimes W\left(z^{2} \int_{S^{\prime} / z^{2}}^{T^{\prime} / z^{2}} Q-S_{u} f^{\prime} d u\right) \Phi_{F}^{c}\right\rangle \mid \leq
\end{aligned}
$$

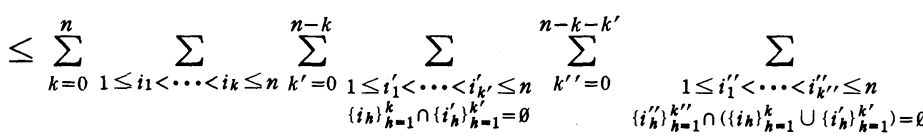

$$
\begin{aligned}
& \sum_{k^{\prime \prime \prime}=0}^{n-k-k^{\prime}-k^{\prime \prime}} \sum_{1 \leq i_{1}^{\prime \prime \prime}<\cdots<i_{k^{\prime \prime \prime}}^{\prime \prime \prime} \leq n} \\
& \left\{i_{h}^{\prime \prime \prime}\right\}_{h=1}^{k^{\prime \prime \prime}} \cap\left(\left\langle i i_{h}\right\}_{h=1}^{k} \cup\left\{i_{h}^{\prime}\right\}_{h=1}^{k^{\prime}} \cup\left\{i_{h}^{\prime \prime}\right\}_{h=1}^{\prime \prime \prime}\right)=\varnothing \\
& \sum_{m=0}^{n-k-k^{\prime}-k^{\prime \prime}-k^{\prime \prime \prime}} \sum_{m^{\prime}=0}^{k^{\prime} \wedge\left(n-k^{\prime}-k^{\prime \prime}-k^{\prime \prime \prime}-m\right)} \sum_{d^{\prime}=0}^{k^{\prime} \wedge\left(k+k^{\prime \prime}\right)} \sum_{d^{\prime \prime}=0}^{k^{\prime \prime} \wedge\left(k-k^{\prime \prime}-d^{\prime}\right)} \sum_{d=0}^{d^{\prime}}\left(d^{\prime}-\bar{d}\right) !
\end{aligned}
$$

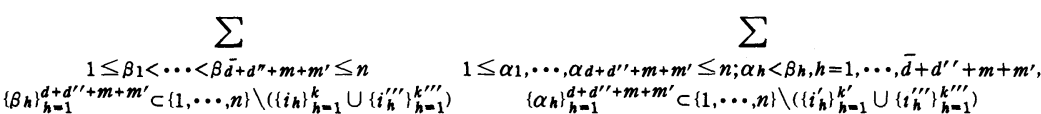

$$
\begin{aligned}
& C_{1}^{2\left(n-m-m^{\prime}-d^{\prime}-d^{\prime \prime}\right)} \cdot z^{2\left(n-m-m^{\prime}-d^{\prime}-d^{\prime \prime}+k^{\prime}+k^{\prime \prime}\right)} \cdot \int_{0}^{t / z^{2}} d t_{1} \int_{0}^{t_{1}} d t_{t} \cdots \int_{0}^{t_{n-1}} d t_{n} \\
& \prod_{h=1}^{\bar{d}+d^{\prime \prime}+m+m^{\prime}}\left|\left\langle P S_{t_{\alpha},} g_{1-\varepsilon\left(\alpha_{h}\right)}, P S_{t_{\beta}, g_{\varepsilon\left(\beta_{h}\right)}}\right\rangle\right| \\
& 1<W\left(z \int_{S / z^{2}}^{T / z^{2}} Q+S_{u} f d u\right) \Phi_{F} \otimes W\left(z^{2} \int_{S / z^{2}}^{T / z^{2}} Q^{-} S_{u} f d u\right) \Phi_{F}^{\iota}, \\
& \left.W\left(z \int_{S^{\prime} / z^{2}}^{T^{\prime} / z^{2}} Q+S_{u} f^{\prime} d u\right) \Phi_{F} \otimes W\left(z^{2} \int_{S^{\prime} / z^{2}}^{T^{\prime} / z^{2}} Q-S_{u} f^{\prime} d u\right) \Phi_{F}^{\iota}\right\rangle \mid
\end{aligned}
$$

where, the factor $\left(d^{\prime}-\bar{d}\right)$ is due to the fact that all the elements in

$$
\left\{p_{h}\right\}_{h=1}^{m} \bigcup\left\{p_{h}^{\prime}\right\}_{h=1}^{m^{\prime}} \bigcup\left\{\tilde{p}_{h}^{\prime}\right\}_{h=1}^{d^{\prime}} \bigcup\left\{\tilde{p}_{h}^{\prime \prime}\right\}_{h=1}^{d^{\prime \prime}} \backslash\left\{\alpha_{h}\right\}_{h=1}^{\bar{d}+b^{\prime \prime}+m+m^{\prime}}
$$

being chose freely and $P=Q_{+}$or $Q^{-}$.

In the following, for each $n \in \mathbf{N}$ and $\varepsilon \in\{0,1\}^{n}$, we denote the left hand side 
of $(5.1)$ by $\Delta(n, \varepsilon, t)$ then, one has

THEOREM (5.1). For each $n \in \mathbf{N}$,

$$
\Delta(n, \varepsilon, t) \leq n^{5} \cdot 8^{n} \max _{0 \leq m \leq n}\left(\|g\|_{s}+o_{z}(1)\right)^{2 m} t^{n-m}
$$

where, $o_{z}(1)$ tends to zero as $z \rightarrow 0$ and $\|g\|_{s}^{2}$ is defined in (1.15).

Proof. Put

$$
f(t):=\sum_{\varepsilon \in\{0,1\}} \max _{P=Q_{+, c^{-1} Q^{-}}}\left|\left\langle P g_{\varepsilon}, S t P g_{\varepsilon}\right\rangle\right|
$$

then,

$$
\int_{-\infty}^{\infty} f(t) d t=\sum_{\varepsilon \in\{0,1\}} \max _{P=Q+, \iota^{-1} Q^{-}} \int_{-\infty}^{\infty}\left|\left\langle P g_{\varepsilon}, S_{t} P g_{\varepsilon}\right\rangle\right| d t
$$

this is controlled by

$$
2 \cdot\|g\|_{s}^{2}+o_{z}(1)
$$

for $z$ small enough. Moreover, $f$ is a bounded symmetric function, therefore using Lemma (9.1) of [0] to the quantity

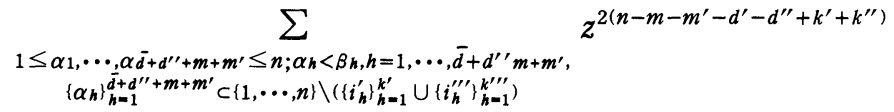

$$
\begin{aligned}
& \int_{0}^{t / z^{2}} d t_{1} \int_{0}^{t_{1}} d t_{2} \cdots \int_{0}^{t_{n-1}} d t_{n}{ }^{\bar{d}+d^{\prime \prime}+m+m^{\prime}} \prod_{h=1}\left|\left\langle S_{t_{\alpha}} P g_{1-\varepsilon\left(\alpha_{h}\right)}, S_{t_{t_{B}}} P g_{\varepsilon\left(\beta_{h}\right)}\right\rangle\right|
\end{aligned}
$$

we find that

$$
\begin{aligned}
& \Delta(n, \varepsilon, t) \leq \sum_{k=0}^{n} \sum_{1 \leq i_{1}<\cdots<i_{k} \leq n} \sum_{k^{\prime}=0}^{n-k} \sum_{1 \leq i_{1}^{\prime}<\cdots<i_{k^{\prime}} \leq n} \sum_{k^{\prime \prime}=0}^{n-k-k^{\prime}} \sum_{1 \leq i_{1}^{\prime \prime}<\cdots<i_{k^{\prime \prime}}^{\prime \prime} \leq n} \\
& \left\{i_{h}\right\}_{h=1}^{k} \cap\left\{i_{h}^{\prime}\right\}_{h=1}^{k^{\prime}=\varnothing} \quad\left\{i_{h}^{\prime \prime}\right\}_{h=1}^{k^{\prime \prime}} \cap\left(\left\{i_{h}\right\}_{h=1}^{k} \cup\left\{i_{h}^{\prime}\right\}_{h=1}^{k^{\prime}}\right)=\varnothing
\end{aligned}
$$

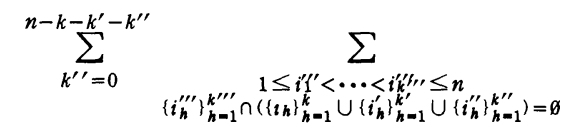

$$
\begin{aligned}
& \sum_{m=0}^{n-k-k^{\prime}-k^{\prime \prime}-k^{\prime \prime \prime}} \sum_{m^{\prime}=0}^{k^{\prime} \wedge\left(n-k^{\prime}-k^{\prime \prime}-k^{\prime \prime \prime}-m\right)} \sum_{d^{\prime}=0}^{k^{\prime} \wedge\left(k+k^{\prime \prime}\right)} \sum_{d^{\prime \prime}=0}^{k^{\prime \prime} \wedge\left(k+k^{\prime \prime}-d^{\prime}\right)} \sum_{\bar{d}=0}^{d^{\prime}} \\
& \sum \quad 2^{n-k-k^{\prime \prime \prime}}\left(d^{\prime}-\bar{d}\right) ! \cdot c_{1}^{2\left(n-m-m^{\prime}-d^{\prime}-d^{\prime \prime}\right)} \cdot z^{2\left(k^{\prime}+k^{\prime \prime}+\bar{d}-d^{\prime}\right)} \\
& \begin{array}{l}
1 \leq \beta_{1}<\cdots<\beta \bar{d}+d^{\prime \prime}+m+m^{\prime} \leq n \\
+m+m^{\prime} \subset\left\{1, \cdots, n \backslash \backslash\left(\left\{i_{h}\right\}_{h=1}^{k} \cup\left\{i_{h}^{\prime \prime \prime}\right\}_{h=1}^{k \prime \prime}\right)\right.
\end{array} \\
& \frac{t^{n-\bar{d}-d^{\prime \prime}-m-m^{\prime}}}{\left(n-\bar{d}-d^{\prime \prime}-m-m^{\prime}\right) !} \cdot\left(\|g\|_{s}+o_{z}(1)\right)^{2\left(\bar{d}+d^{\prime \prime}+m+m^{\prime}\right)}
\end{aligned}
$$


where,

$$
c_{1}:=1+\max _{\varepsilon \in\{0,1\} ; Q^{\prime}=-\frac{1}{2} \beta H, 1 ; F=f, f^{\prime}} \int_{-\infty}^{\infty}\left|\left\langle g_{\varepsilon}, S_{t} Q^{\prime} F\right\rangle\right| d t
$$

Notice that the set

$$
\left\{p_{h}\right\}_{h=1}^{m} \bigcup\left\{p_{h}^{\prime}\right\}_{h=1}^{m^{\prime}} \bigcup\left\{\bar{p}_{h}^{\prime}\right\}_{h=1}^{d^{\prime}} \bigcup\left\{\bar{p}_{h}^{\prime \prime}\right\}_{h=1}^{d^{\prime \prime}}
$$

labels the annihilation operator which are used to produce scalar products, therefore we should have at least $m+m^{\prime}+d^{\prime}+d^{\prime \prime}$ creators, but each fixed time corresponds to two operators, this implies that

$$
m+m^{\prime}+d^{\prime}+d^{\prime \prime}+m+m^{\prime}+d^{\prime}+d^{\prime \prime} \leq 2 n
$$

i.e.

$$
m+m^{\prime}+d^{\prime}+d^{\prime \prime} \leq n
$$

therefore,

$$
\begin{aligned}
& \frac{\left(d^{\prime}-d\right) ! \cdot c_{1}^{2\left(n-m-m^{\prime}-d^{\prime}-d^{\prime \prime}\right)} \cdot z^{2\left(k^{\prime}+k^{\prime \prime}+d-d^{\prime}\right)}}{\left(n-\bar{d}-d^{\prime \prime}-m-m^{\prime}\right) !} \\
& \leq \frac{\left(d^{\prime}-d\right) ! \cdot c_{1}^{2\left(n-m-m^{\prime}-d^{\prime}-d^{\prime \prime}\right)}}{\left(n-d^{\prime}-d^{\prime \prime}-m-m^{\prime}+\left(d^{\prime}-\bar{d}\right)\right) !} \\
& \leq \frac{c_{1}^{2\left(n-m-m^{\prime}-d^{\prime}-d^{\prime \prime}\right)}}{\left(n-d^{\prime}-d^{\prime \prime}-m-m^{\prime}\right) !}=O(1) .
\end{aligned}
$$

Moreover, the sum

$$
\sum_{m=0}^{n-k-k^{\prime}-k^{\prime \prime}-k^{\prime \prime \prime}} \sum_{m^{\prime}=0}^{k^{\prime} \wedge\left(n-k^{\prime}-k^{\prime \prime}-k^{\prime \prime \prime}-m\right)} \sum_{d^{\prime}=0}^{k^{\prime} \wedge\left(k+k^{\prime \prime}\right)} \sum_{d^{\prime \prime}=0}^{k^{\prime \prime} \wedge\left(k+k^{\prime \prime}-d^{\prime}\right)} \sum_{d=0}^{d^{\prime}}
$$

is less than $n^{5}$ and

$$
\begin{aligned}
& \sum_{k=0}^{n} \sum_{1 \leq i_{1}<\cdots<i k \leq n} \sum_{k^{\prime}=0}^{n-k} \sum_{1 \leq i_{1}^{\prime}<\cdots<i_{k^{\prime}} \leq n} \sum_{k^{\prime \prime}=0}^{n-k-k^{\prime}} \sum_{1 \leq i_{1}^{\prime \prime}<\cdots<i_{k^{\prime \prime \prime}} \leq n} \\
& \left\{i_{h}\right\}_{h=1}^{k} \cap\left\{i_{h}^{\prime} h_{h=1}^{k^{\prime}}=\varnothing \quad\left\{i_{h}^{\prime \prime}\right\}_{h=1}^{k^{\prime \prime}} \cap\left(\{i h\}_{h=1}^{k} \cup\left\{i_{h}^{\prime}\right\}_{h=1}^{k^{\prime}}\right)=\varnothing\right. \\
& \sum_{k^{\prime \prime \prime}=0}^{n-k-k^{\prime}-k^{\prime \prime}} \sum_{\substack{1 \leq i_{1}^{\prime \prime \prime}<\cdots<i_{k^{\prime \prime \prime \prime}}^{\prime \prime \prime} \leq n \\
\left\{i _ { h } ^ { \prime \prime \prime } k _ { h = 1 } ^ { \prime \prime } \cap \left(\{ t h \} _ { h = 1 } ^ { k } \cup \left\{i_{h}^{\prime} k_{h=1}^{\prime \prime} \cup\left\{i_{h}^{\prime \prime}\right\}_{h=1}^{\left.k^{\prime \prime}\right)=\varnothing}\right.\right.\right.}} 2^{n-k-k^{\prime \prime \prime}}
\end{aligned}
$$

$$
\begin{gathered}
=\sum_{k=0}^{n} 2^{n-k}\left(\begin{array}{l}
n \\
k
\end{array}\right) \sum_{k^{\prime}=0}^{n-k}\left(\begin{array}{c}
n-k \\
k^{\prime}
\end{array}\right) \sum_{k^{\prime \prime}=0}^{n-k-k^{\prime}}\left(n-\underset{k^{\prime \prime}}{k-k^{\prime}}\right) \sum_{k^{\prime \prime \prime}=0}^{n-k-k^{\prime}-k^{\prime \prime}}\left(\begin{array}{c}
n-\underset{k^{\prime \prime \prime}}{k}-k^{\prime}-k^{\prime \prime} \\
k^{\prime}
\end{array}\right) 2^{-k^{\prime \prime \prime}} \\
=\sum_{k=0}^{n} 2^{n-k}\left(\begin{array}{l}
n \\
k
\end{array}\right) \sum_{k^{\prime}=0}^{n-k}\left(\begin{array}{c}
n-k \\
k^{\prime}
\end{array}\right) \sum_{k^{\prime \prime}=0}^{n-k-k^{\prime}}\left(\begin{array}{c}
n-\underset{k}{k-k^{\prime}} \\
k^{\prime \prime}
\end{array}\right)(3 / 2)^{n-k-k^{\prime}-k^{\prime \prime}}
\end{gathered}
$$




$$
\begin{aligned}
& =\sum_{k=0}^{n} 2^{n-k}\left(\begin{array}{l}
n \\
k
\end{array}\right) \sum_{k^{\prime}=0}^{n-k}\left(\begin{array}{c}
n-k \\
k^{\prime}
\end{array}\right)(5 / 2)^{n-k-k^{\prime}} \\
= & \sum_{k=0}^{n} 2^{n-k}\left(\begin{array}{l}
n \\
k
\end{array}\right)(7 / 2)^{n-k}=\sum_{k=0}^{n}\left(\begin{array}{l}
n \\
k
\end{array}\right) 7^{n-k}=8^{n}
\end{aligned}
$$

so the proof is finished.

Theorem (5.2). For each $r \in\{0, \pm 1, \pm 2\}, \sigma \in\{0,1\}$,

$$
\begin{gathered}
\mid\left\langle W\left(z \int_{S / z^{2}}^{T / z^{2}} Q_{+} S_{u} f d u\right) \Phi_{F} \otimes W\left(z^{2} \int_{S / z^{2}}^{T / z^{2}} Q-S_{u} f d u\right) \Phi_{F}^{\iota}\right. \\
\frac{1}{z^{2}} \cdot z^{|r|} A_{r}\left(S_{t / z^{2}} g_{\sigma}, \mathrm{S}_{t / z^{2}} g_{1-\sigma}\right) \int_{0}^{t / z^{2}} d t_{1} \int_{0}^{t_{1}} d t_{2} \cdots \int_{0}^{t n-1} d t_{n}\left(\mathrm{I}_{n}^{\varepsilon}+\mathrm{II}_{n}^{\varepsilon}\right) \cdot \\
\left.\cdot W\left(z \int_{S^{\prime} / z^{2}}^{T^{\prime} / z^{2}} Q+S_{u} f^{\prime} d u\right) \Phi_{F} \otimes W\left(z^{2} \int_{S^{\prime} / z^{2}}^{T^{\prime} / z^{2}} Q-S_{u} f^{\prime} d u\right) \Phi_{F}^{\iota}\right\rangle \mid \leq \\
\leq n^{6} \cdot 8^{n} \max _{0 \leq m \leq n}\left(\|g\|_{s}+o_{z}(1)\right)^{2 m} t^{n-m} .
\end{gathered}
$$

Proof. The proof is almost the same as ones of Theorem (5.1) and the difference is only replacing

by

$$
\frac{t^{n-\bar{d}-d^{\prime \prime}-m-m^{\prime}}}{\left(n-\bar{d}-d^{\prime \prime}-m-m^{\prime}\right) !}
$$

$$
\frac{t^{n-\bar{d}-d^{\prime \prime}-m-m^{\prime}-1}}{\left(n-\bar{d}-d^{\prime \prime}-m-m^{\prime}-1\right) !}
$$

\section{\$6. The low density limit and its properties}

In the section we shall

i) prove that the limit (1.13) exists and formulate its explicit form;

ii) find a differential (or integro) equation satisfied by the limit (1.13).

Theorem (6.1). For each $g_{0}, g_{1}, \in K, t \geq 0, D \in B\left(H_{0}\right)$ satisfying (1.15), and each $f, f^{\prime} \in K, S, T, S^{\prime}, T^{\prime \prime}, \in \mathbf{R}, u, v \in H_{0}$, the low density limit

(6.1) $\lim _{z \rightarrow 0}\left\langle u \otimes W\left(z \int_{S / z^{2}}^{T / z^{2}} S_{u} f d u\right) \Phi_{z}, U_{t / z^{2}} v \otimes W\left(z \int_{S^{\prime} / z^{2}}^{T^{\prime} / z^{2}} S_{u} f^{\prime} d u\right) \Phi_{z}\right\rangle$ exists and is equal to

$$
\sum_{n=0}^{\infty} \sum_{\alpha \in\{0,1\}}\left\langle u, D_{\varepsilon(1)} \cdots D_{\varepsilon(n)} v\right\rangle
$$




$$
\begin{aligned}
& \sum_{k=0}^{[n / 2]} \sum_{1 \leq i_{1}^{\prime}<\cdots<i_{k}<i_{k}^{\prime} \leq n} \sum_{l=1}^{n-2 k} \sum_{\substack{1 \leq j_{1}<\cdots<j_{l} \leq n \\
\left\{j_{h}\right\}_{h=1}^{l} \in\{1, \cdots, n\} \backslash\left(i_{h}, i_{h}^{\prime}\right\}_{h=1}^{k}}} \\
& \sum_{m=\Sigma_{h=1}^{k}\left(i_{h}^{\prime}-i_{h}-1\right)}^{n-2 k-l} \sum_{\substack{1 \leq q_{1}<\cdots<q_{m} \leq n \\
\left\{q_{h}\right\}_{h=1}^{m} \subset\{1, \cdots, n\} \backslash\left\{\left(i_{h}, i_{h}^{\prime}\right\}_{h=1}^{k} \cup\left\{j_{h}^{\prime}\right\}_{h=1}^{\prime} ;\left\{q_{h}-1\right\}_{h=1}^{m} \cap\left(\left\{i_{h}^{\prime}, i_{h}^{\prime}-1\right\}_{h=1}^{k}=\varnothing\right.\right.}}
\end{aligned}
$$

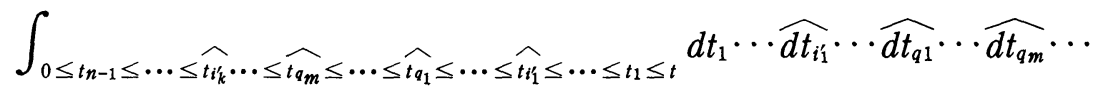

$$
\begin{aligned}
& \widehat{d t_{i^{\prime}}} \cdots d t_{n} \\
& \prod_{h=1}^{k} M\left(\varepsilon_{1}, i_{h}, i_{h}^{\prime},\left\{q_{h}\right\}_{h=1}^{m}\right) \cdot N\left(\varepsilon_{1}\left\{i_{h+1}, i_{h}^{\prime}\right\}_{h=0}^{k},\left\{q_{h}\right\}_{h=1}^{m}\right) \\
& \prod_{h=1}^{l}\left\langle g_{\varepsilon\left(j_{h}\right)}, g_{1-\varepsilon\left(j_{h}\right)}\right\rangle\left\langle W\left(\chi_{|S, T|} \otimes f\right) \Psi, W\left(\chi_{\left|S^{\prime}, T^{\prime}\right|} \otimes f^{\prime}\right) \Psi\right\rangle
\end{aligned}
$$

where, $M\left(\varepsilon, i_{h}, i_{h}^{\prime},\left\{q_{h}\right\}_{h=1}^{m}\right\}, h=1, \ldots, m$ and $N\left(\varepsilon,\left\{i_{h+1}, i_{h}^{\prime}\right\}_{h=0}^{k},\left\{q_{h}\right\}_{h=1}^{m}\right)$ are defined in (4.61) and (4.65).

Proof.

$$
\begin{gathered}
\left\langle u \otimes W\left(z \int_{S / z^{2}}^{T / z^{2}} S_{u} f d u\right) \Phi_{z}, U_{t / z^{2}} v \otimes W\left(z \int_{S^{\prime} / z^{2}}^{T^{\prime} / z^{2}} S_{u} f^{\prime} d u\right) \Phi_{z}\right\rangle \\
=\sum_{n=0}^{\infty} \sum_{\varepsilon \in(0,1\}}\left\langle u, D_{\varepsilon(1)} \cdots D_{\varepsilon(n)} v\right\rangle \\
\left\langle u \otimes W\left(z \int_{S / z^{2}}^{T / z^{2}} S_{u} f d u\right) \Phi_{z}, \int_{0}^{t / z^{2}} d t_{1} \int_{0}^{t} d t_{2} \cdots \int_{0}^{t n-1} d t_{n}\right. \\
\left.A^{+}\left(S_{t_{1}} g_{\varepsilon(1)}\right) A\left(S_{t_{1}} g_{1-\varepsilon(1)}\right) \cdots A^{+}\left(S_{t n} g_{\varepsilon(n)}\right) A\left(S_{t n} g_{1-\varepsilon(n)}\right) v \otimes W\left(z \int_{S^{\prime} / z^{2}}^{T^{\prime} / z^{2}} S_{u} f^{\prime} d u\right) \Phi_{z}\right\rangle .
\end{gathered}
$$

By the uniform estimate,

$$
\begin{gathered}
\text { (6.4) } \lim _{z \rightarrow 0}\left\langle u \otimes W\left(z \int_{S / z^{2}}^{T / z^{2}} S_{u} f d u\right) \Phi_{z}, U_{t / z^{2}} v \otimes W\left(z \int_{S^{\prime} / z^{2}}^{T^{\prime} / z^{2}} S_{u} f^{\prime} d u\right) \Phi_{z}\right\rangle \\
=\sum_{n=0}^{\infty} \sum_{\alpha \in(0,1)}\left\langle u, D_{\varepsilon(1)} \cdots D_{\varepsilon(n)} u\right\rangle \\
\lim _{z \rightarrow 0}\left\langle u \otimes W\left(z \int_{S / z^{2}}^{T / z^{2}} S_{u} f d u\right) \Phi_{z}, \int_{0}^{t^{\prime} / z^{2}} d t_{1} \int_{0}^{t_{1}} d t_{2} \cdots \int_{0}^{t_{n-1}} d t_{n}\right. \\
\left.A^{+}\left(S_{t_{1}} g_{\varepsilon(1)}\right) A\left(S_{t_{1}} g_{1-\varepsilon(1)}\right) \cdots A^{+}\left(S_{t n} g_{\varepsilon(n)}\right) A\left(S_{t n} g_{1-\varepsilon(n)}\right) v \otimes W\left(z \int_{S^{\prime} / z^{2}}^{T^{\prime} / z^{2}} S_{u} f^{\prime} d u\right) \Phi_{z}\right\rangle .
\end{gathered}
$$

Applying Theorem (4.12) to (6.4), we finish the proof at once.

This theorem shows that for each $u, v \in H_{0}$, there exists a $G(t) \in H_{0}$, such that 


$$
\begin{gathered}
\langle u, G(t)\rangle \\
=\lim _{z \rightarrow 0}\left\langle u \otimes W\left(z \int_{S / z^{2}}^{T / z^{2}} S_{u} f d u\right) \Phi_{z}, U_{t / z^{2}} v \otimes W\left(z \int_{S^{\prime} / z^{2}}^{T^{\prime} / z^{2}} S_{u} f^{\prime} d u\right) \Phi_{z}\right\rangle .
\end{gathered}
$$

For each $z>0$, defining

$$
\begin{gathered}
\left\langle u, G_{z}(t)\right\rangle \\
:=\left\langle u \otimes W\left(z \int_{S / z^{2}}^{T / z^{2}} S_{u} f d u\right) \Phi_{z}, U_{t / z^{2}} v \otimes W\left(z \int_{S^{\prime} / z^{2}}^{T^{\prime} / z^{2}} S_{u} f^{\prime} d u\right) \Phi_{z}\right\rangle
\end{gathered}
$$

then

$$
\begin{gathered}
\langle u, G(0)\rangle=\lim _{z \rightarrow 0}\left\langle u, G_{z}(0)\right\rangle= \\
=\lim _{z \rightarrow 0}\left\langle u \otimes W\left(z \int_{S / z^{2}}^{T / z^{2}} S_{u} f d u\right) \Phi_{z}, v \otimes W\left(z \int_{S^{\prime} / z^{2}}^{T^{\prime} / z^{2}} S_{u} f^{\prime} d u\right) \Phi_{z}\right\rangle \\
=\langle u, v\rangle\left\langle W\left(\chi_{|S, T|} \otimes f\right) \Psi, W\left(\chi_{\left|S^{\prime}, T^{\prime}\right|} \otimes f^{\prime}\right) \Psi\right\rangle .
\end{gathered}
$$

So,

$$
\begin{aligned}
\langle u, G(t)\rangle= & \lim _{z \rightarrow 0}\left\langle u, G_{z}(t)\right\rangle=\langle u, G(0)\rangle \\
& +\lim _{z \rightarrow 0} \int_{0}^{t} \frac{d}{d s}\left\langle u, G_{z}(s)\right\rangle d s .
\end{aligned}
$$

Moreover, for each $z>0, n \in \mathbf{N}$,

$$
\begin{gathered}
\mid \frac{d}{d s}\left\langle u \otimes W\left(z \int_{S / z^{2}}^{T / z^{2}} S_{u} f d u\right) \Phi_{z}, \int_{0}^{s / z^{2}} d t_{1} \int_{0}^{t_{1}} d t_{2} \cdots \int_{0}^{t_{n-1}} d t_{n}\right. \\
\left.V\left(t_{1}\right) \cdots V\left(t_{n}\right) v \otimes W\left(z \int_{S^{\prime} / z^{2}}^{T^{\prime} / z^{2}} S_{u} f^{\prime} d u\right) \Phi_{z}\right\rangle \mid= \\
=\mid\left\langle u \otimes W\left(z \int_{S / z^{2}}^{T / z^{2}} S_{u} f d u\right) \Phi_{z}, \int_{0}^{s / z^{2}} d t_{2} \int_{0}^{t_{2}} d t_{2} \cdots \int_{0}^{t_{n-1}} d t_{n}\right. \\
\left.\frac{1}{z^{2}} V\left(s / z^{2}\right) V\left(t_{2}\right) \cdots V\left(t_{n}\right) v \otimes W\left(z \int_{S^{\prime} / z^{2}}^{T^{\prime} / z^{2}} S_{u} f^{\prime} d u\right) \Phi_{z}\right\rangle \mid .
\end{gathered}
$$

Hence from Theorem (5.2) follows that

$$
\begin{gathered}
\frac{d}{d s}\left\langle u, G_{z}(s)\right\rangle=\sum_{n=1}^{\infty}\left\langle u \otimes W\left(z \int_{S / z^{2}}^{T / z^{2}} S_{u} f d u\right) \Phi_{z},\right. \\
\frac{d}{d s} \int_{0}^{s / z^{2}} d t_{1} \int_{0}^{t_{1}} d t_{2} \cdots \int_{0}^{t n-1} d t_{n}(-i)^{n} V\left(t_{1}\right) \cdots V\left(t_{n}\right) v \otimes \\
\left.W\left(z \int_{S^{\prime} / z^{2}}^{T^{\prime} / z^{2}} S_{u} f^{\prime} d u\right) \Phi_{z}\right\rangle
\end{gathered}
$$




$$
\begin{gathered}
=\sum_{n-1}^{\infty} \frac{-i}{z^{2}}\left\langle u \otimes W\left(z \int_{S / z^{2}}^{T / z^{2}} S_{u} f d u\right) \Phi_{z},\right. \\
V\left(s / z^{2}\right) \int_{0}^{s / z^{2}} d t_{2} \int_{0}^{t_{2}} d t_{3} \cdots \int_{0}^{t_{n-1}} d t_{n}(-i)^{n-1} V\left(t_{2}\right) \cdots V\left(t_{n}\right) \\
\left.v \otimes W\left(z \int_{S^{\prime} / z^{2}}^{T^{\prime} / z^{2}} S_{u} f^{\prime} d u\right) \Phi_{z}\right\rangle \\
=\frac{-i}{z^{2}}\left\langle u \otimes W\left(z \int_{S / z^{2}}^{T / z^{2}} S_{u} f d u\right) \Phi_{z}, V\left(s / z^{2}\right) U_{s / z^{2}} v \otimes W\left(z \int_{S^{\prime} / z^{2}}^{T^{\prime} / z^{2}} S_{u} f^{\prime} d u\right) \Phi_{z}\right\rangle .
\end{gathered}
$$

Moreover, by expanding $V$ to the sum of the products of creation and annihilation operators according to (1.15) and (1.10), we find that

$$
\begin{gathered}
\frac{d}{d s}\left\langle u, G_{z}(s)\right\rangle=\frac{1}{z^{2}}\left\langle u \otimes W\left(z \int_{S / z^{2}}^{T / z^{2}} S_{u} Q+f d u\right) \Phi_{F}\right. \\
\left.\otimes W\left(z^{2} \int_{S / z^{2}}^{T / z^{2}} Q^{-} S_{u} f d u\right) \Phi_{F}^{c}\right\rangle \\
\sum_{\varepsilon \in\{0,1\}} D_{\varepsilon} \otimes\left(A^{+}\left(S_{S / z^{2}} Q+g_{\varepsilon}\right) A\left(S_{S / z^{2}} Q+g_{1-\varepsilon}\right) \otimes 1\right.
\end{gathered}
$$

$$
\begin{gathered}
z \cdot A^{+}\left(S_{s / z^{2}} Q+g_{\varepsilon}\right) \otimes A^{+}\left(Q^{-} S_{s / z^{2}} g_{1-\varepsilon}\right)+z \cdot A\left(S_{s / z^{2}} Q+g_{1-\varepsilon}\right) \otimes A\left(Q^{-} S_{s / z^{2}} g_{\varepsilon}\right) \\
\left.+z^{2} 1 \otimes\left(A^{+}\left(Q^{-} S_{s / z^{2}} g_{1-\varepsilon}\right) A\left(Q^{-} S_{s / z^{2}} g_{\varepsilon}\right)+\left\langle Q^{-} g_{\varepsilon}, Q^{-} g_{1-\varepsilon}\right\rangle_{\iota}\right)\right) U_{s / z^{2}} \\
\left.v \otimes W\left(z \int_{S^{\prime} / z^{2}}^{T^{\prime} / z^{2}} S_{u} Q_{+} f^{\prime} d u\right) \Phi_{F} \otimes W\left(z^{2} \int_{S^{\prime} / z^{2}}^{T^{\prime} / z^{2}} S_{u} Q^{-} f^{\prime} d u\right) \Phi_{F}^{c}\right\rangle \\
:=\frac{1}{z^{2}} \mathrm{I}_{z}+\frac{1}{z} \mathrm{II}_{z}+\frac{1}{z} \mathrm{III}_{z}+\mathrm{IV}_{z} .
\end{gathered}
$$

Now our first step is to deal with $\frac{1}{z^{2}} \mathrm{I}_{z}$. We split $\frac{1}{z^{2}} \mathrm{I}_{z}$ into a sum of two terms, one of them corresponds to the case of the creator $A^{+}\left(S_{s / z^{2}} Q+g_{\varepsilon}\right)$ acting on the coherent vector $W\left(z \int_{S / z^{2}}^{T / z^{2}} S_{u} Q+f d u\right) \Phi_{F}$ and the annihilation operator $A^{+}\left(S_{S / z^{2}} Q_{+} g_{1-\varepsilon}\right)$ acting on the coherent vector $W\left(z \int_{S^{\prime} / z^{2}}^{T} S_{u} Q_{+} f^{\prime} d u\right) \Phi_{F}$; the other one corresponds to the case of the creator $A^{+}\left(S_{s / z^{2}} Q+g_{\varepsilon}\right)$ acting on the coherent vector $W\left(z \int_{S / z^{2}}^{T / z^{2}} S_{u} Q_{+} f d u\right) \Phi_{F}$ and the annihilation operator $A^{+}\left(S_{s / z^{2}} Q_{+}\right.$ $\left.g_{1-\varepsilon}\right)$ commuting with the operator $U_{s / z^{2}}$, i.e.

$$
\frac{1}{z^{2}} \mathrm{I}_{z}=\sum_{\varepsilon \in\{0.1\}}\left\langle D_{\varepsilon}^{+} u \otimes W\left(z \int_{S / z^{2}}^{T / z^{2}} S_{u} Q+f d u\right) \Phi_{F} \otimes W\left(z^{2} \int_{S / z^{2}}^{T / z^{2}} Q^{-} S_{u} f d u\right) \Phi_{F}^{\iota}\right.
$$




$$
\begin{gathered}
\int_{S / z^{2}}^{T / z^{2}}\left\langle S_{u} Q+f, S_{S / z^{2}} Q+g_{\varepsilon}\right\rangle d u \cdot \int_{S^{\prime} / z^{2}}^{T^{\prime} / z^{2}}\left\langle S_{S / z^{2}} Q+g_{1-\varepsilon}, S_{u} Q+f^{\prime}\right\rangle d u \cdot U_{S / z^{2}} \\
\left.v \otimes W\left(z \int_{S^{\prime} / z^{2}}^{T^{\prime} / z^{2}} S_{u} Q+f^{\prime} d u\right) \Phi_{F} \otimes W\left(z^{2} \int_{S^{\prime} / z^{2}}^{T^{\prime} / z^{2}} Q-S_{u} f^{\prime} d u\right) \Phi_{F}^{\iota}\right\rangle+ \\
+\sum_{\varepsilon \in\{0,1\}}\left\langle D_{\varepsilon}^{+} u \otimes W\left(z \int_{S / z^{2}}^{T / z^{2}} S_{u} Q+f d u\right) \Phi_{F} \otimes W\left(z^{2} \int_{S / z^{2}}^{T / z^{2}} Q^{-} S_{u} f d u\right) \Phi_{F}^{\iota}\right. \\
\frac{1}{z} \int_{S / z^{2}}^{T / z^{2}}\left\langle S_{u} Q_{+} f, S_{S / z^{2}} Q+g_{\varepsilon}\right\rangle d u \cdot\left[1 \otimes A\left(S_{S^{\prime} / z^{2}} Q+g_{1-\varepsilon}\right) \otimes, U_{S / z^{2}}\right] \\
\left.v \otimes W\left(z \int_{S^{\prime} / z^{2}}^{T^{\prime} / z^{2}} S_{u} Q_{+} f^{\prime} d u\right) \Phi_{F} \otimes W\left(z^{2} \int_{S^{\prime} / z^{2}}^{T^{\prime} / z^{2}} Q-S_{u} f^{\prime} d u\right) \Phi_{F}^{\iota}\right\rangle \\
:=\mathrm{I}_{z}(a)+\mathrm{I}_{z}(b) .
\end{gathered}
$$

It is obvious that

$$
\lim _{z \rightarrow 0} \mathrm{I}_{z}(a)=\sum_{\varepsilon \in\{0,1\}} \chi_{\mid S, T\}}(s)\left(f \mid g_{\varepsilon}\right) \cdot \chi_{\left[S^{\prime}, T^{\prime}\right]}(s)\left(g_{1-\varepsilon} \mid f^{\prime}\right)\left\langle D_{\varepsilon}^{+} u, G(s)\right\rangle \text { a.e. }
$$

Moreover denoting for each $n \in \mathbf{N}$,

$$
\begin{gathered}
\mathrm{I}_{z}(n, a):=\sum_{\varepsilon \in\{0,1\}}\left\langle D_{\varepsilon}^{+} u \otimes W\left(z \int_{S / z^{2}}^{T / z^{2}} S_{u} Q+f d u\right) \Phi_{F} \otimes W\right. \\
\times\left(z^{2} \int_{S / z^{2}}^{T / z^{2}} Q^{-} S_{u} f d u\right) \Phi_{F}^{\iota} \\
\int_{S / z^{2}}^{T / z^{2}}\left\langle S_{u} Q_{+} f, S_{S / z^{2}} Q+g_{\varepsilon}\right\rangle d u \cdot \int_{S^{\prime} / z^{2}}^{T^{\prime} / z^{2}}\left\langle S_{S / z^{2}} Q+g_{1-\varepsilon}, S_{u} Q_{+} f^{\prime}\right\rangle d u \\
\int_{0}^{s / z^{2}} d t_{1} \int_{0}^{t_{1}} d t_{2} \cdots \int_{0}^{t n-1} d t_{n} V\left(t_{1}\right) \cdots V\left(t_{n}\right) \\
\left.v \otimes W\left(z \int_{S^{\prime} / z^{2}}^{T^{\prime} / z^{2}} S_{u} Q+f^{\prime} d u\right) \Phi_{F} \otimes W\left(z^{2} \int_{S^{\prime} / z^{2}}^{T^{\prime} / z^{2}} Q^{-} S_{u} f^{\prime} d u\right) \Phi_{F}^{<}\right\rangle
\end{gathered}
$$

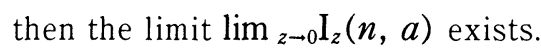

In order to obtain the limit

$$
\lim _{z \rightarrow 0} I_{z}(b)
$$

we need the following

Lemma (6.2). The limit

$\lim _{z \rightarrow 0} \sum_{\varepsilon \in\{0,1\}}\left\langle D_{\varepsilon}^{+} u \otimes W\left(z \int_{S / z^{2}}^{T / z^{2}} S_{u} Q_{+} f d u\right) \Phi_{F} \otimes W\left(z^{2} \int_{S / z^{2}}^{T / z^{2}} Q^{-} S_{u} f d u\right) \Phi_{F}^{\iota}\right.$, 


$$
\begin{gathered}
\frac{1}{z}\left[1 \otimes A\left(S_{s / z^{2}} Q+g_{1-\varepsilon}\right) \otimes 1, U_{s / z^{2}}\right] \\
\left.v \otimes W\left(z \int_{S^{\prime} / z^{2}}^{T^{\prime} / z^{2}} S_{u} Q+f^{\prime} d u\right) \Phi_{F} \otimes W\left(z^{2} \int_{S^{\prime} / z^{2}}^{T^{\prime} / z^{2}} Q^{-} S_{u} f^{\prime} d u\right) \Phi_{F}^{\iota}\right\rangle
\end{gathered}
$$

exists.

Proof. By the uniform estimate (Theorem (5.2)), it is enough to show that for each $n \in \mathbf{N}$,

$$
\begin{gathered}
\lim _{z \rightarrow 0} \sum_{\varepsilon \in\{0,1\}}\left\langle D_{\varepsilon}^{+} u \otimes W\left(z \int_{S / z^{2}}^{T / z^{2}} S_{u} Q_{+} f d u\right) \Phi_{F} \otimes W\left(z^{2} \int_{S / z^{2}}^{T / z^{2}} Q^{-} S_{u} f d u\right) \Phi_{F}^{\iota},\right. \\
\int_{0}^{s / z^{2}} d t_{1} \cdots \int_{0}^{t n-1} d t_{n} \frac{1}{z}(-i)^{n}\left[1 \otimes A\left(S_{S / z^{2}} Q_{+} g_{1-\varepsilon}\right) \otimes 1, V\left(t_{1}\right) \cdots V\left(t_{n}\right)\right] \\
\left.v \otimes W\left(z \int_{S^{\prime} / z^{2}}^{T^{\prime} / z^{2}} S_{u} Q_{+} f^{\prime} d u\right) \Phi_{F} \otimes W\left(z^{2} \int_{S^{\prime} / z^{2}}^{T^{\prime} / z^{2}} Q^{-} S_{u} f^{\prime} d u\right) \Phi_{F}^{\iota}\right\rangle
\end{gathered}
$$

exists.

Denoting the limit (6.16) by

$$
\lim _{z \rightarrow 0} J(n, z, s)
$$

let us prove the lemma by induction.

First of all,

$$
\begin{gathered}
J(1, z, s)=\sum_{\varepsilon, \varepsilon(1) \in\{0,1\}} \\
\left\langle D_{\varepsilon}^{+} u \otimes W\left(z \int_{S / z^{2}}^{T / z^{2}} S_{u} Q_{+} f d u\right) \Phi_{F} \otimes W\left(z^{2} \int_{S / z^{2}}^{T / z^{2}} Q^{-} S_{u} f d u\right) \Phi_{F}^{\iota}\right. \\
\int_{0}^{s / z^{2}} d t_{1} D_{\varepsilon(1)} \otimes \frac{1}{z}\left[A\left(S_{s / z^{2}} Q_{+} g_{1-\varepsilon}\right) \otimes 1\right. \\
\left(A^{+}\left(S_{t_{1}} Q_{+} g_{\varepsilon(1)}\right) A\left(S_{t_{1}} Q_{+} g_{1-\varepsilon(1)}\right) \otimes 1+\right.
\end{gathered}
$$

$z\left(A^{+}\left(S_{t_{1}} Q_{+} g_{\varepsilon(1)}\right) \otimes A^{+}\left(Q^{-} S_{t_{1}} g_{1-\varepsilon(1)}\right)+A\left(S_{t_{1}} Q_{+} g_{1-\varepsilon(1)}\right) \otimes A\left(Q^{-} S_{t_{1}} g_{\varepsilon(1)}\right)\right)+$

$\left.+z^{2} 1 \otimes\left(\left\langle Q^{-} S_{t_{1}} g_{\varepsilon(1)}, Q^{-} S_{t_{1}} g_{1-\varepsilon(1)}\right\rangle+A^{+}\left(Q^{-} S_{t_{1}} g_{1-\varepsilon(1)}\right) A\left(Q^{-} S_{t_{1}} g_{\varepsilon(1)}\right)\right)\right]$

$$
\begin{gathered}
\left.v \otimes W\left(z \int_{S^{\prime} / z^{2}}^{T^{\prime} / z^{2}} S_{u} Q_{+} f^{\prime} d u\right) \Phi_{F} \otimes W\left(z^{2} \int_{S^{\prime} / z^{2}}^{T^{\prime} / z^{2}} Q-S_{u} f^{\prime} d u\right) \Phi_{F}^{L}\right\rangle \\
:=J_{1}(1, z, s)+J_{2}(1, z, s)+J_{3}(1, z, s)+J_{4}(1, z, s)
\end{gathered}
$$

Notice that $J_{3}(1, z, s)=J_{4}(1, z, s)=0$, now let us investigate the first two 
terms.

$$
J_{1}(1, z, s)=\sum_{\varepsilon, \varepsilon(1) \in\{0,1\}}\left\langle D_{\varepsilon(1)}^{+} D_{\varepsilon}^{+} u, v\right\rangle
$$

$$
\begin{gathered}
\left\langle W\left(z \int_{S / z^{2}}^{T / z^{2}} S_{u} Q+f d u\right) \Phi_{F}, \int_{0}^{s / z^{2}} d t_{1} \frac{1}{z}\left[A\left(S_{S / z^{2}} Q+g_{1-\varepsilon}\right), A^{+}\left(S_{t_{1}} Q+g_{\varepsilon(1)}\right)\right]\right. \\
\left.A\left(S_{t_{1}} Q+g_{1-\varepsilon(1)}\right) W\left(z \int_{S^{\prime} / z^{2}}^{T^{\prime} / z^{2}} S_{u} Q+f^{\prime} d u\right) \Phi_{F}\right\rangle \\
\left\langle W\left(z \int_{S / z^{2}}^{T / z^{2}} Q^{-} S_{u} f d u\right) \Phi_{F}^{c}, W\left(z^{2} \int_{S^{\prime} / z^{2}}^{T^{\prime} / z^{2}} Q^{-} S_{u} f^{\prime} d u\right) \Phi_{F}^{c}\right\rangle_{\iota} \\
=\sum_{\varepsilon, \varepsilon(1) \in\{0,1\}}\left\langle D_{\varepsilon(1)}^{+} D_{\varepsilon}^{+} u, v\right\rangle
\end{gathered}
$$

$\int_{0}^{s / z^{2}} d t_{1}\left\langle S_{s / z^{2}} Q+g_{1-\varepsilon}, S_{t_{1}} Q+g_{\varepsilon(1)}\right\rangle \int_{S^{\prime} / z^{2}}^{T / / z^{2}}\left\langle S_{t_{1}} Q+g_{1-\varepsilon(1)}, S_{u} Q+f^{\prime}\right\rangle d u$

$$
\begin{gathered}
\left\langle W\left(z \int_{S / z^{2}}^{T / z^{2}} S_{u} Q_{+} f d u\right) \Phi_{F}, W\left(z \int_{S^{\prime} / z^{2}}^{T^{\prime} / z^{2}} S_{u} Q_{+} f^{\prime} d u\right) \Phi_{F}\right\rangle \\
\left\langle W\left(z^{2} \int_{S / z^{2}}^{T / z^{2}} Q^{-} S_{u} f d u\right) \Phi_{F}^{\iota}, W\left(z^{2} \int_{S^{\prime} / z^{2}}^{T^{\prime} / z^{2}} Q^{-} S_{u} f^{\prime} d u\right) \Phi_{F}^{\iota}\right\rangle_{\iota}
\end{gathered}
$$

With the change of variable

$$
t_{1}-s / z^{2}=s_{1}
$$

the right hand side of (6.19) becomes

$$
\sum_{\varepsilon, \varepsilon(1) \in\{0,1\}}\left\langle D_{\varepsilon(1)}^{+} D_{\varepsilon}^{+} u, v\right\rangle
$$

$$
\begin{gathered}
\int_{-s / z^{2}}^{0} d s_{1}\left\langle Q+g_{1-\varepsilon}, S_{s_{1}} Q+g_{\varepsilon(1)}\right\rangle \int_{S^{\prime} / z^{2}}^{T / z^{2}}\left\langle S_{S_{1}+s / z^{2}} Q+g_{1-\varepsilon(1)}, S_{u} Q+f^{\prime}\right\rangle d u \\
\left\langle W\left(z \int_{S / z^{2}}^{T / z^{2}} S_{u} f d u\right) \Phi_{z}, W\left(z \int_{S^{\prime} / z^{2}}^{T^{\prime} / z^{2}} S_{u} f^{\prime} d u\right) \Phi_{z}\right\rangle .
\end{gathered}
$$

Again with the change of variable

$$
u-s_{1}-s / z^{2}=v
$$

(6.20) becomes

$$
\sum_{\varepsilon, \varepsilon(1) \in\{0,1\}}\left\langle D_{\varepsilon(1)}^{+} D_{\varepsilon}^{+} u, v\right\rangle
$$

$$
\begin{gathered}
\int_{-s / z^{2}}^{0} d s_{1}\left\langle Q_{+} g_{1-\varepsilon}, S_{s_{1}} Q_{+} g_{\varepsilon(1)}\right\rangle \int_{\left(S^{\prime}-s\right) / z^{2}-s_{1}}^{\left(T^{\prime}-s\right) / z^{2}-s_{1}}\left\langle Q_{+} g_{1-\varepsilon(1)}, S_{u} Q_{+} f^{\prime}\right\rangle d v \\
\left\langle W\left(z \int_{S / z^{2}}^{T / z^{2}} S_{u} f d u\right) \Phi_{z}, W\left(z \int_{S^{\prime} / z^{2}}^{T^{\prime} / z^{2}} S_{u} f^{\prime} d u\right) \Phi_{z}\right\rangle
\end{gathered}
$$


which tends to

$$
\begin{gathered}
\sum_{\varepsilon, \varepsilon(1) \in\{0,1\}}\left\langle D_{\varepsilon(1)}^{+} D_{\varepsilon}^{+} u, v\right\rangle \int_{-\infty}^{0} d s_{1}\left\langle g_{1-\varepsilon}, S_{s_{1}} g_{\varepsilon(1)}\right\rangle\left(g_{1-\varepsilon(1)} \mid f^{\prime}\right) \cdot \chi_{\left[S^{\prime}, T^{\prime}\right]}(s) \\
\left\langle W\left(\chi_{[S, T]} \otimes f\right) \Psi, W\left(\chi_{\left[S^{\prime}, T^{\prime}\right]} \otimes f^{\prime}\right) \Psi\right\rangle
\end{gathered}
$$

Moreover,

$$
J_{2}(1, z, s)=\sum_{\varepsilon, \varepsilon(1) \in\{0,1\}}
$$

$$
\begin{gathered}
\left\langle D_{\varepsilon(1)}^{+} D_{\varepsilon}^{+} u \otimes W\left(z \int_{S / z^{2}}^{T / z^{2}} S_{u} Q+f d u\right) \Phi_{F} \otimes W\left(z^{2} \int_{S / z^{2}}^{T / z^{2}} Q-S_{u} f d u\right) \Phi_{F}^{\iota},\right. \\
\int_{0}^{s / z^{2}} d t_{1} 1 \otimes\left[A\left(S_{S / z^{2}} Q+g_{1-\varepsilon}\right), A^{+}\left(S_{t_{1}} Q+g_{\varepsilon(1)}\right)\right] \otimes A^{+}\left(Q^{-} S_{t_{1}} g_{1-\varepsilon(1)}\right) \\
\left.v \otimes W\left(z \int_{S^{\prime} / z^{2}}^{T^{\prime} / z^{2}} S_{u} Q+f^{\prime} d u\right) \Phi_{F} \otimes W\left(z^{2} \int_{S^{\prime} / z^{2}}^{T^{\prime} / z^{2}} Q^{-} S_{u} f^{\prime} d u\right) \Phi_{F}^{\iota}\right\rangle \\
=\sum_{\varepsilon, \varepsilon(1) \in\{0,1\}}\left\langle D_{\varepsilon(1)}^{+} D_{\varepsilon}^{+} u, v\right\rangle \\
z^{2} \int_{S / z^{2}}^{T / z^{2}}\left\langle Q-S_{u} f, Q^{-} S_{t_{1}} g_{1-\varepsilon(1)}\right\rangle, d u \int_{0}^{s / z^{2}} d t_{1}\left\langle S_{S / z^{2}} Q+g_{1-\varepsilon}, S_{t_{1}} Q+g_{\varepsilon(1)}\right\rangle \\
\left\langle W\left(z \int_{S / z^{2}}^{T / z^{2}} S_{u} f d u\right) \Phi_{Q}, W\left(z \int_{S^{\prime} / z^{2}}^{T^{\prime} / z^{2}} S_{u} f^{\prime} d u\right) \Phi_{Q}\right\rangle .
\end{gathered}
$$

With the same change of variables (6.19) and (6.21), one knows that the right hand side of (6.23) is equal to $z^{2} \cdot O(1)$ and tends to zero.

Assuming that the limit (6.16) exists for $n$, let see the situation in $n+1$. By the same arguments as in (6.17), one can write $J(n+1, z, s)$ as a sum of $J_{1}(n+1, z, s)$ and $J_{2}(n+1, z, s)$, where

$$
\begin{gathered}
J_{1}(n+1, z, s):=\sum_{\varepsilon, \varepsilon(1) \in\{0,1\}} \\
\left\langle D_{\varepsilon}^{+} u \otimes W\left(z \int_{S / z^{2}}^{T / z^{2}} S_{u} Q+f d u\right) \Phi_{F} \otimes W\left(z^{2} \int_{S / z^{2}}^{T / z^{2}} Q^{-} S_{u} f d u\right) \Phi_{F}^{\iota},\right. \\
\int_{0}^{s / z^{2}} d t_{1} \cdots \int_{0}^{t_{n}} d t_{n+1} D_{\varepsilon(1)} \otimes \frac{1}{z}\left[A\left(S_{S / z^{2}} Q+g_{1-\varepsilon}\right), A^{+}\left(S_{t_{1}} Q+g_{\varepsilon(1)}\right)\right] \\
A\left(S_{t_{1}} Q+g_{1-\varepsilon(1)}\right) \otimes 1 V\left(t_{2}\right) \cdots V\left(t_{n+1}\right)(-i)^{n} \\
\left.v \otimes W\left(z \int_{S^{\prime} / z^{2}}^{T^{\prime} / z^{2}} S_{u} Q_{+} f^{\prime} d u\right) \Phi_{F} \otimes W\left(z^{2} \int_{S^{\prime} / z^{2}}^{T^{\prime} / z^{2}} Q^{-} S_{u} f^{\prime} d u\right) \Phi_{F}^{<}\right\rangle
\end{gathered}
$$

and

$$
J_{2}(n+1, z, s):=\sum_{\varepsilon, \varepsilon(1) \in\{0,1\}}
$$




$$
\begin{gathered}
\left\langle D_{\varepsilon}^{+} u \otimes W\left(z \int_{S / z^{2}}^{T / z^{2}} S_{u} Q+f d u\right) \Phi_{F} \otimes W\left(z^{2} \int_{S / z^{2}}^{T / z^{2}} Q-S_{u} f d u\right) \Phi_{F}^{\iota}\right. \\
\int_{0}^{s / z^{2}} d t_{1} \int_{0}^{t_{1}} d t_{2} \cdots \int_{0}^{t_{n}} d t_{n+1} D_{\varepsilon(1)} \otimes\left[A\left(S_{S / z^{2}} Q+g_{1-\varepsilon}\right), A^{+}\left(S_{t 1} Q+g_{\varepsilon(1)}\right)\right] \\
1 \otimes 1 \otimes A^{+}\left(Q^{-} S_{t_{1}} g_{1-\varepsilon(1)}\right) V\left(t_{2}\right) \cdots V\left(t_{n+1}\right)(-i)^{n} \\
\left.v \otimes W\left(z \int_{S^{\prime} / z^{2}}^{T^{\prime} / z^{2}} S_{u} Q_{+} f^{\prime} d u\right) \Phi_{F} \otimes W\left(z^{2} \int_{S^{\prime} / z^{2}}^{T^{\prime} / z^{2}} Q^{-} S_{u} f^{\prime} d u\right) \Phi_{F}^{\iota}\right\rangle
\end{gathered}
$$

In (6.25), we apply the creator $A^{+}\left(Q^{-} S_{t_{1}} g_{1-\varepsilon(1)}\right)$ to the coherent vector

$$
W\left(z^{2} \int_{S / z^{2}}^{T / z^{2}} Q^{-} S_{u} f d u\right) \Phi_{F}^{\iota}
$$

and change of variable

$$
t_{1}-s / z^{2}=: t^{\prime}
$$

one finds that the module of $J_{2}(n+1, z, s)$ is less than or equal to

$$
\sum_{\varepsilon, \varepsilon(1) \in\{0,1\}} \mid\left\langle D_{\varepsilon(1)}^{+} D_{\varepsilon}^{+} u \otimes W\left(z \int_{S / z^{2}}^{T / z^{2}} S_{u} Q+f d u\right) \Phi_{F}\right.
$$

$$
\begin{gathered}
\otimes W\left(z^{2} \int_{S / z^{2}}^{T / z^{2}} Q^{-} S_{u} f d u\right) \Phi_{F}^{\iota}, \int_{-s / z^{2}}^{0} d t^{\prime}\left\langle Q+g_{1-\varepsilon}, S_{t^{\prime}} Q_{+} g_{\varepsilon(1)}\right\rangle \\
z_{2} \int_{S / z^{2}}^{T / z^{2}}\left\langle Q^{-} S_{u} f, Q^{-} S_{t^{\prime}-s / z^{2}} g_{1-\varepsilon(1)}\right\rangle_{\iota} \int_{0}^{t^{\prime}+s / z^{2}} d t_{2} \cdots \int_{0}^{t_{n}} d t_{n+1} V\left(t_{2}\right) \cdots V\left(t_{n+1}\right) \\
\left.v \otimes W\left(z \int_{S^{\prime} / z^{2}}^{T^{\prime} / z^{2}} S_{u} Q_{+} f^{\prime} d u\right) \Phi_{F} \otimes W\left(z^{2} \int_{S^{\prime} / z^{2}}^{T^{\prime} / z^{2}} Q^{-} S_{u} f^{\prime} d u\right) \Phi_{F}^{\iota}\right\rangle \mid \\
=z^{2} \cdot O(1) \longrightarrow 0 .
\end{gathered}
$$

In the formula (6.24), the term $\left[A\left(S_{s / z^{2}} Q+g_{1-\varepsilon}\right), A^{+}\left(S_{t_{1}} Q+g_{\varepsilon(1)}\right]\right.$ is equal to the scalar product $\left\langle S_{s / z^{2}} Q+g_{1-\varepsilon}, S_{t_{1}} Q+g_{\varepsilon(1)}\right\rangle$ and the operator

$$
1 \otimes A\left(S_{t_{1}} Q_{+} g_{1-\varepsilon(1)}\right) \otimes 1 V\left(t_{2}\right) \cdots V\left(t_{n+1}\right)
$$

is equal to the sum of the following two terms:

$$
V\left(t_{2}\right) \cdots V\left(t_{n+1}\right) 1 \otimes A\left(S_{t_{1}} Q+g_{1-\varepsilon(1)}\right) \otimes 1
$$

and

$$
\left[1 \otimes A\left(S_{t_{1}} Q+g_{1-\varepsilon(1)}\right) \otimes 1, V\left(t_{2}\right) \cdots \mathrm{V}\left(t_{n+1)}\right]\right.
$$

therefore 


$$
\begin{gathered}
J_{1}(n+1, z, s)=\sum_{\varepsilon, \varepsilon(1) \in\{0,1\}} \\
\left\langle D_{\varepsilon}^{+} u \otimes W\left(z \int_{S / z^{2}}^{T / z^{2}} S_{u} Q+f d u\right) \Phi_{F} \otimes W\left(z^{2} \int_{S / z^{2}}^{T / z^{2}} Q^{-} S_{u} f d u\right) \Phi_{F}^{\iota},\right. \\
(-i)^{n} \int_{0}^{s / z^{2}} d t_{1} \cdots \int_{0}^{t n} d t_{n+1} D_{\varepsilon(1)} \otimes \frac{1}{z}\left\langle S_{S / z^{2}} Q+g_{1-\varepsilon}, S_{t_{1}} Q+g_{\varepsilon(1)}\right\rangle \\
\left(V\left(t_{2}\right) \cdots V\left(t_{n+1}\right) 1 \otimes A\left(S_{t_{1}} Q+g_{1-\varepsilon(1)}\right) \otimes 1+\right. \\
\left.+\left[1 \otimes A\left(S_{t_{1}} Q+g_{1-\varepsilon(1)}\right) \otimes 1, V\left(t_{2}\right) \cdots V\left(t_{n+1}\right)\right]\right) \\
\left.v \otimes W\left(z \int_{S^{\prime} / z^{2}}^{T^{\prime} / z^{2}} S_{u} Q_{+} f^{\prime} d u\right) \Phi_{F} \otimes W\left(z^{2} \int_{S^{\prime} / z^{2}}^{T^{\prime} / z^{2}} Q^{-} S_{u} f^{\prime} d u\right) \Phi_{F}^{\iota}\right\rangle .
\end{gathered}
$$

By applying the annihilation operator $A\left(S_{t_{1}} Q+g_{1-\varepsilon(1)}\right)$ on the coherent vector

$$
W\left(z \int_{S^{\prime} / z^{2}}^{T^{\prime} / z^{2}} S_{u} Q_{+} f^{\prime} d u\right) \Phi_{F}
$$

the first term of (6.29) becomes

$$
\begin{gathered}
\sum_{\varepsilon, \varepsilon(1) \in\{0,1\}}\left\langle D_{\varepsilon(1)}^{+} D_{\varepsilon}^{+} u \otimes W\left(z \int_{S / z^{2}}^{T / z^{2}} S_{u} Q_{+} f d u\right) \Phi_{F} \otimes W\left(z^{2} \int_{S / z^{2}}^{T / z^{2}} Q-S_{u} f d u\right) \Phi_{F}^{\iota},\right. \\
\quad \int_{0}^{s / z^{2}} d t_{1} \cdots \int_{0}^{t_{n}} d t_{n+1} \frac{1}{z}\left\langle S_{S / z^{2}} Q+g_{1-\varepsilon}, S_{t_{1}} Q+g_{\varepsilon(1)}\right\rangle \\
(-i)^{n} V\left(t_{2}\right) \cdots V\left(t_{n+1}\right) z \int_{S^{\prime} / z^{2}}^{T^{\prime} / z^{2}}\left\langle S_{t_{1}} Q_{+} g_{1-\varepsilon(1)}, S_{u} Q_{+} f^{\prime}\right\rangle d u \\
\left.v \otimes W\left(z \int_{S^{\prime} / z^{2}}^{T^{\prime} / z^{2}} S_{u} Q_{+} f^{\prime} d u\right) \Phi_{F} \otimes W\left(z^{2} \int_{S^{\prime} / z^{2}}^{T^{\prime} / z^{2}} Q-S_{u} f^{\prime} d u\right) \Phi_{F}^{\iota}\right\rangle .
\end{gathered}
$$

With the change of variable (6.26) and using Lemma (6.3) of [2], (6.30) becomes

$$
\begin{gathered}
\sum_{\varepsilon, \varepsilon(1) \in\{0,1\}}\left\langle D_{\varepsilon(1)}^{+} D_{\varepsilon}^{+} u \otimes W\left(z \int_{S / z^{2}}^{T / z^{2}} S_{u} Q_{+} f d u\right) \Phi_{F} \otimes W\left(z^{2} \int_{S / z^{2}}^{T / z^{2}} Q^{-} S_{u} f d u\right) \Phi_{F}^{\iota},\right. \\
\int_{-s / z^{2}}^{0} d t^{\prime}\left\langle Q_{+} g_{1-\varepsilon}, S_{t^{\prime}} Q_{+} g_{\varepsilon(1)}\right\rangle \int_{0}^{s / z^{2}} d t_{2} \cdots \int_{0}^{t n} d t_{n+1} V\left(t_{2}\right) \cdots V\left(t_{n+1}\right)(-i)^{n} \\
\int_{s^{\prime} / z^{2}}^{T^{\prime} / z^{2}}\left\langle S_{t^{\prime}+s / z^{2}} Q_{+} g_{1-\varepsilon(1)}, S_{u} Q_{+} f^{\prime}\right\rangle d u \\
\left.v \otimes W\left(z \int_{S^{\prime} / z^{2}}^{T^{\prime} / z^{2}} S_{u} Q_{+} f^{\prime} d u\right) \Phi_{F} \otimes W\left(z^{2} \int_{S^{\prime} / z^{2}}^{T^{\prime} / z^{2}} Q-S_{u} f^{\prime} d u\right) \Phi_{F}^{\iota}\right\rangle .
\end{gathered}
$$

Thus the conclusion of Section 4 and the fact that 


$$
\begin{gathered}
\int_{-s / z^{2}}^{0} d t^{\prime}\left\langle Q_{+} g_{1-\varepsilon}, S_{t^{\prime}} Q+g_{\varepsilon(1)}\right\rangle \int_{s^{\prime} / z^{2}}^{T^{\prime} / z^{2}}\left\langle S_{t^{\prime}+3 / z^{2}} Q+g_{1-\varepsilon(1)}, S_{u} Q+f^{\prime}\right\rangle d u \\
\longrightarrow \int_{-\infty}^{0} d t^{\prime}\left\langle g_{1-\varepsilon}, S_{t^{\prime}} g_{\varepsilon(1)}\right\rangle \chi_{\left[S^{\prime}, T^{\prime}\right]}(s)\left(g_{1-\varepsilon(1)} \mid f^{\prime}\right), \text { a.e. }
\end{gathered}
$$

shows that the limit of (6.30) exists.

Similar arguments show that by induction the limit of the second term of (6.30) exists.

Summing up we finish the proof.

LEMma (6.3).

$$
\begin{aligned}
\lim _{z \rightarrow 0} \sum_{\varepsilon \in\{0,1\}}\left\langle D_{\varepsilon}^{+} u \otimes W\right. & \left.\left(z \int_{S / z^{2}}^{T / z^{2}} S_{u} Q_{+} f d u\right) \Phi_{F} \otimes W\left(z^{2} \int_{S / z^{2}}^{T / z^{2}} Q^{-} S_{u} f d u\right) \Phi_{F}^{\iota}\right\rangle \\
& {\left[1 \otimes 1 \otimes A\left(Q^{-} S_{S / z^{2}} g_{\varepsilon}\right), U_{S / z^{2}}\right] }
\end{aligned}
$$$$
\left.v \otimes W\left(z \int_{S^{\prime} / z^{2}}^{T^{\prime} / z^{2}} S_{u} Q+f^{\prime} d u\right) \Phi_{F} \otimes W\left(z^{2} \int_{S^{\prime} / z^{2}}^{T^{\prime} / z^{2}} Q^{-} S_{u} f^{\prime} d u\right) \Phi_{F}^{\iota}\right\rangle=0 .
$$

Proof. By the uniform estimate (Theorem (5.2)) it is enough to show that for each $n \in \mathbf{N}$,

$$
\begin{aligned}
& \sum_{\varepsilon\{0,1\}}\left\langle D_{\varepsilon}^{+} u \otimes W\left(z \int_{S / z^{2}}^{T / z^{2}} S_{u} Q_{+} f d u\right) \Phi_{F} \otimes W\left(z^{2} \int_{S / z^{2}}^{T / z^{2}} Q^{-} S_{u} f d u\right) \Phi_{F}^{c}\right. \\
& \int_{0}^{s / z^{2}} d t_{1} \cdots \int_{0}^{t n-1} d t_{n}(-i)^{n}\left[1 \otimes 1 \otimes A\left(Q^{-} S_{S / z^{2}} g_{\varepsilon}\right), V\left(t_{1}\right) \cdots V\left(t_{n}\right)\right] \\
& \left.v \otimes W\left(z \int_{S^{\prime} / z^{2}}^{T^{\prime} / z^{2}} S_{u} Q+f^{\prime} d u\right) \Phi_{F} \otimes W\left(z^{2} \int_{S^{\prime} / z^{2}}^{T^{\prime} / z^{2}} Q^{-} S_{u} f^{\prime} d u\right) \Phi_{F}^{\iota}\right\rangle \longrightarrow 0
\end{aligned}
$$

The left hand side of (6.34) is equal to

$$
\begin{gathered}
\sum_{\varepsilon, \varepsilon(1) \in\{0,1\}}\left\langle D_{\varepsilon(1)}^{+} D_{\varepsilon}^{+} u \otimes W\left(z \int_{S / z^{2}}^{T / z^{2}} S_{u} Q+f d u\right) \Phi_{F} \otimes W\left(z^{2} \int_{S / z^{2}}^{T / z^{2}} Q^{-} S_{u} f d u\right) \Phi_{F}^{c},\right. \\
\int_{0}^{s / z^{2}} d t_{1} \cdots \int_{0}^{t_{n-1}} d t_{n}(-i)^{n-1}\left(z\left\langle Q^{-} S_{S / z^{2}} g_{\varepsilon}, Q^{-} S_{t_{1}} g_{\varepsilon(1)}\right\rangle_{\iota}\right. \\
1 \otimes A^{+}\left(Q^{-} S_{t_{1}} g_{1-\varepsilon(1)}\right) \otimes 1 V\left(t_{2}\right) \cdots V\left(t_{n}\right)+ \\
+z^{2}\left\langle Q^{-} S_{S / z^{2}} g_{\varepsilon}, Q^{-} S_{t_{1}} g_{1-\varepsilon(1)}\right\rangle_{\iota} V\left(t_{2}\right) \cdots V\left(t_{n}\right) 1 \otimes A\left(Q^{-} S_{t_{1}} g_{\varepsilon(1)} \otimes 1+\right. \\
\left.+z^{2}\left\langle Q^{-} S_{S / z^{2}} g_{\varepsilon}, Q^{-} S_{t_{1}} g_{1-\varepsilon(1)}\right\rangle_{\iota}\left[1 \otimes 1 \otimes A\left(Q^{-} S_{t_{1}} g_{\varepsilon(1)}\right) V\left(t_{2}\right) \cdots V\left(t_{n}\right)\right]\right)
\end{gathered}
$$




$$
\left.v \otimes W\left(z \int_{S^{\prime} / z^{2}}^{T^{\prime} / z^{2}} S_{u} Q_{+} f^{\prime} d u\right) \Phi_{F} \otimes W\left(z^{2} \int_{S^{\prime} / z^{2}}^{T^{\prime} / z^{2}} Q^{-} S_{u} f^{\prime} d u\right) \Phi_{F}^{\iota}\right\rangle
$$

The first two terms of (6.35) are both equal to

$$
z^{2} \cdot O(1) \longrightarrow 0
$$

and moreover the same arguments as those in the proof of Lemma (6.2) show that the third term of (6.35) is $z^{2} O(1)$, therefore we end our proof.

We can obtain more from the proof of Lemma (6.2), in fact the proof shows how does get the limit (6.14). Now let us realize it.

By definition

$$
\begin{gathered}
\mathrm{I}_{z}(b)=\sum_{n=1}^{\infty} \sum_{\varepsilon \in\{0,1\}} \\
\left\langle D_{\varepsilon}^{+} u \otimes W\left(z \int_{S / z^{2}}^{T / z^{2}} S_{u} Q_{+} f d u\right) \Phi_{F} \otimes W\left(z^{2} \int_{S / z^{2}}^{T / z^{2}} Q^{-} S_{u} f d u\right) \Phi_{F}^{\iota}\right. \\
\int_{0}^{s / z^{2}} d t_{1} \cdots \int_{0}^{t n-1} d t_{n} \int_{S / z^{2}}^{T / z^{2}}\left\langle S_{u} Q_{+} f, S_{s / z^{2}} Q_{+} g_{\varepsilon}\right\rangle d u \\
\frac{1}{z}(-i)^{n}\left[1 \otimes A\left(S_{S / z^{2}} Q_{+} g_{1-\varepsilon}\right) \otimes 1, V\left(t_{1}\right) \cdots V\left(t_{n}\right)\right] \\
\left.v \otimes W\left(z \int_{S^{\prime} / z^{2}}^{T^{\prime} / z^{2}} S_{u} Q_{+} f^{\prime} d u\right) \Phi_{F} \otimes W\left(z^{2} \int_{S^{\prime} / z^{2}}^{T^{\prime} / z^{2}} Q-S_{u} f^{\prime} d u\right) \Phi_{F}^{\iota}\right\rangle .
\end{gathered}
$$

By Lemma (6.2), the fact that

$$
\int_{S / z^{2}}^{T / z^{2}}\left\langle S_{u} Q+f, S_{s / z^{2}} Q+g_{\varepsilon}\right\rangle d u \longrightarrow \chi_{[S, T]}(s) \cdot\left(f \mid g_{\varepsilon}\right), \text { a.e. }
$$

and an argument similar to the one used in $(6.24), \ldots,(6.30)$, we know that the limit of $\mathrm{I}_{z}(b)$ is equal to limit of

$$
\begin{gathered}
\sum_{n=0}^{\infty} \sum_{\varepsilon, \varepsilon(1) \in\{0,1\}}\left\langle D_{\varepsilon(1)}^{+} D_{\varepsilon}^{+} u \otimes W\left(z \int_{S / z^{2}}^{T / z^{2}} S_{u} Q+f d u\right) \Phi_{F} \otimes W\left(z^{2} \int_{S / z^{2}}^{T / z^{2}} Q^{-} S_{u} f d u\right) \Phi_{F}^{\iota},\right. \\
\chi[S, T](s) \cdot\left(f \mid g_{\varepsilon}\right) \int_{-s / z^{2}}^{0} d t^{\prime}\left\langle Q+g_{1-\varepsilon}, S_{t^{\prime}} Q+g_{\varepsilon(1)}\right\rangle \\
(-i)^{n} \int_{0}^{s / z^{2}} d t_{2} \cdots \int_{0}^{t_{n}} d t_{n+1} V\left(t_{2}\right) \cdots V\left(t_{n-1}\right) \\
\times \int_{S^{\prime} / z^{2}}^{T^{\prime} / z^{2}}\left\langle S_{t^{\prime}+s / z^{2}} Q_{+} g_{1-\varepsilon(1)}, S_{u} Q_{+} f^{\prime}\right\rangle d u \\
\left.v \otimes W\left(z \int_{S^{\prime} / z^{2}}^{T^{\prime} / z^{2}} S_{u} Q_{+} f^{\prime} d u\right) \Phi_{F} \otimes W\left(z^{2} \int_{S^{\prime} / z^{2}}^{T^{\prime} / z^{2}} Q^{-} S_{u} f^{\prime} d u\right) \Phi_{F}^{\iota}\right\rangle
\end{gathered}
$$




$$
\begin{gathered}
+\sum_{n=1}^{\infty} \sum_{\varepsilon, \varepsilon(1) \in\{0,1\}}\left\langle D_{\varepsilon(1)}^{+} D_{\varepsilon}^{+} u \otimes W\left(z \int_{S / z^{2}}^{T / z^{2}} S_{u} Q_{+} f d u\right) \Phi_{F} \otimes\right. \\
W\left(z^{2} \int_{S / z^{2}}^{T / z^{2}} Q^{-} S_{u} f d u\right) \Phi_{F}^{\iota}, \\
\chi_{[S, T\}}(s) \cdot\left(f \mid g_{\varepsilon}\right) \int_{0}^{s / z^{2}} d t_{2} \cdots \int_{0}^{t n} d t_{n+1} \int_{-s / z^{2}}^{0} d t^{\prime}\left\langle Q+g_{1-\varepsilon}, S_{t^{\prime}} Q+g_{\varepsilon(1)}\right\rangle \\
(-i)^{n} \frac{1}{z}\left[1 \otimes A\left(S_{t^{\prime}+s / z^{2}} Q_{+} g_{1-\varepsilon(1)}\right) \otimes 1, V\left(t_{2}\right) \cdots V\left(t_{n+1}\right)\right] \\
\left.v \otimes W\left(z \int_{S^{\prime} / z^{2}}^{T^{\prime} / z^{2}} S_{u} Q+f^{\prime} d u\right) \Phi_{F} \otimes W\left(z^{2} \int_{S^{\prime} / z^{2}}^{T^{\prime} / z^{2}} Q^{-} S_{u} f^{\prime} d u\right) \Phi_{F}^{\iota}\right\rangle .
\end{gathered}
$$

Using Lemma (6.3) of [2], we know that the limit of (6.39) is equal to the limit of $(6.40)$

$\sum_{n=0}^{\infty} \sum_{\varepsilon, \varepsilon(1) \in\{0,1\}}\left\langle D_{\varepsilon(1)}^{+} D_{\varepsilon}^{+} u \otimes W\left(z \int_{S / z^{2}}^{T / z^{2}} S_{u} Q_{+} f d u\right) \Phi_{F} \otimes W\left(z^{2} \int_{S / z^{2}}^{T / z^{2}} Q^{-} S_{u} f d u\right) \Phi_{F}^{\epsilon}\right.$,

$$
\begin{aligned}
& \chi_{[S, T]}(s) \cdot\left(f \mid g_{\varepsilon}\right) \int_{-\infty}^{0} d t^{\prime}\left\langle g_{1-\varepsilon}, S_{t^{\prime}} g_{\varepsilon(1)}\right\rangle \\
& (-i)^{n} \int_{0}^{s / z^{2}} d t_{2} \cdots \int_{0}^{t_{n}} d t_{n+1} V\left(t_{2}\right) \cdots V\left(t_{n+1}\right) \chi_{\left[S^{\prime}, T^{\prime}\right]}\left(g_{1-\varepsilon(1)} \mid f^{\prime}\right) \\
& \left.v \otimes W\left(z \int_{S^{\prime} / z^{2}}^{T^{\prime} / z^{2}} S_{u} Q_{+} f^{\prime} d u\right) \Phi_{F} \otimes W\left(z^{2} \int_{S^{\prime} / z^{2}}^{T^{\prime} / z^{2}} Q-S_{u} f^{\prime} d u\right) \Phi_{F}^{\iota}\right\rangle+ \\
& +\sum_{n=1}^{\infty} \sum_{\varepsilon, \varepsilon(1) \in\{0,1\}}\left\langle D_{\varepsilon(1)}^{+} D_{\varepsilon}^{+} u \otimes W\left(z \int_{S / z^{2}}^{T / z^{2}} S_{u} Q_{+} f d u\right) \Phi_{F} \otimes\right. \\
& W\left(z^{2} \int_{S / z^{2}}^{T / z^{2}} Q^{-} S_{u} f d u\right) \Phi_{F}^{\iota} \\
& \chi_{[S, T]}(s) \cdot\left(f \mid g_{\varepsilon}\right) \int_{-\infty}^{0} d t^{\prime}\left\langle g_{1-\varepsilon}, S_{t^{\prime}} g_{\varepsilon(1)}\right\rangle \\
& (-i)^{n} \frac{1}{z} \int_{0}^{s / z^{2}} d t_{2} \cdots \int_{0}^{t_{n}} d t_{n+1}\left[1 \otimes A\left(S_{s / z^{2}} Q_{+} g_{1-\varepsilon(1)}\right) \otimes 1, V\left(t_{2}\right) \cdots V\left(t_{n+1}\right)\right] \\
& \left.v \otimes W\left(z \int_{S^{\prime} / z^{2}}^{T^{\prime} / z^{2}} S_{u} Q_{+} f^{\prime} d u\right) \Phi_{F} \otimes W\left(z^{2} \int_{S^{\prime} / z^{2}}^{T^{\prime} / z^{2}} Q^{-} S_{u} f^{\prime} d u\right) \Phi_{F}^{\iota}\right\rangle \\
& =\sum_{\varepsilon, \varepsilon(1) \in\{0,1\}}\left\langle D_{\varepsilon(1)}^{+} D_{\varepsilon}^{+} u \otimes W\left(z \int_{S / z^{2}}^{T / z^{2}} S_{u} Q_{+} f d u\right) \Phi_{F} \otimes W\left(z^{2} \int_{S / z^{2}}^{T / z^{2}} Q^{-} S_{u} f d u\right) \Phi_{F}^{\iota},\right. \\
& \chi_{[S, T]}(s) \cdot\left(f \mid g_{\varepsilon}\right) \chi_{\left[S^{\prime}, T^{\prime}\right]}\left(g_{1-\varepsilon(1)} \mid f^{\prime}\right)\left(g_{1-\varepsilon} \mid g_{\varepsilon(1)}\right)_{-} \\
& \left.U_{s / z^{2}} v \otimes W\left(z \int_{S^{\prime} / z^{2}}^{T^{\prime} / z^{2}} S_{u} Q+f^{\prime} d u\right) \Phi_{F} \otimes W\left(z^{2} \int_{S^{\prime} / z^{2}}^{T^{\prime} / z^{2}} Q^{-} S_{u} f^{\prime} d u\right) \Phi_{F}^{\iota}\right\rangle
\end{aligned}
$$




$$
\begin{gathered}
+\sum_{\varepsilon \in\{0,1\}}\left\langle D_{1-\varepsilon}^{+} D_{\varepsilon}^{+} u \otimes W\left(z \int_{S / z^{2}}^{T / z^{2}} S_{u} Q_{+} f d u\right) \Phi_{F} \otimes W\left(z^{2} \int_{S / z^{2}}^{T / z^{2}} Q^{-} S_{u} f d u\right) \Phi_{F}^{\iota},\right. \\
\frac{1}{z} \chi_{[S, T]}(s) \cdot\left(f \mid g_{\varepsilon}\right)\left(g_{1-\varepsilon} \mid g_{1-\varepsilon}\right)-\left[1 \otimes A\left(S_{S / z^{2}} Q+g_{1-\varepsilon(1)}\right) \otimes 1, U_{S / z^{2}}\right] \\
\left.v \otimes W\left(z \int_{S^{\prime} / z^{2}}^{T^{\prime} / z^{2}} S_{u} Q_{+} f^{\prime} d u\right) \Phi_{F} \otimes W\left(z^{2} \int_{S^{\prime} / z^{2}}^{T^{\prime} / z^{2}} Q^{-} S_{u} f^{\prime} d u\right) \Phi_{F}^{\iota}\right\rangle .
\end{gathered}
$$

As $z \rightarrow 0$, the first term of the right hand side of (6.40) goes to

$$
\sum_{\varepsilon, \varepsilon(1) \in\{0,1\}}\left\langle D_{\varepsilon^{(}(1)}^{+} D_{\varepsilon}^{+} u, G(s)\right\rangle \chi_{[s, T]}(s) \cdot\left(f \mid g_{\varepsilon}\right) \chi_{\left[S^{\prime}, T^{\prime}\right]}(s)\left(g_{1-\varepsilon(1)} \mid f^{\prime}\right)\left(g_{1-\varepsilon} \mid g_{\varepsilon(1)}\right)_{-}
$$

and the second term of the right hand side of (6.40) is of the same type as $\mathrm{I}_{z}(b)$.

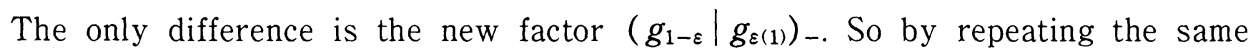
arguments about $I_{z}(b)$ on the second term of the right hand side of (6.40), we obtain the following result:

Lemma (6.4).

$$
\begin{gathered}
\lim _{z \rightarrow 0} I_{z}(b)=\sum_{\varepsilon \in\{0,1\}} \sum_{n=0}^{\infty} \sum_{\sigma \in\{0,1\} n}\left\langle D_{\varepsilon}^{+} D_{\sigma(n)}^{+} \cdots \cdots D_{\sigma(1)}^{+} D_{\varepsilon}^{+} u, G(s)\right\rangle \\
\left(g_{1-\varepsilon} \mid g_{\sigma(1)}\right)_{-} \cdot\left(g_{1-\sigma(1)} \mid g_{\sigma(2)}\right)_{-} \cdots \cdots\left(g_{1-\sigma(n)} \mid g_{\varepsilon}\right)_{-} \\
\quad \chi_{[S, T]}(s) \cdot\left(f \mid g_{\varepsilon}\right) \chi_{\left[S^{\prime}, T^{\prime}\right]}(s) \cdot\left(g_{1-\varepsilon} \mid f^{\prime}\right) \\
+\sum_{\varepsilon \in\{0,1\}} \sum_{n=0}^{\infty} \sum_{\sigma \in\{0,1\}^{n}}\left\langle D_{1-\varepsilon}^{+} D_{\sigma(n)}^{+} \cdots \cdots D_{\sigma(1)}^{+} D_{\varepsilon}^{+} u, G(s)\right\rangle \\
\left(g_{1-\varepsilon} \mid g_{\sigma(1)}\right)_{-} \cdot\left(g_{1-\sigma(1)} \mid g_{\sigma(2)}\right)_{-} \cdots \cdots\left(g_{1-\sigma(n)} \mid g_{1-\varepsilon}\right)_{-} \\
\chi_{[S, T]}(s) \cdot\left(f \mid g_{\varepsilon}\right) \chi_{\left[S^{\prime}, T^{\prime}\right]}(s) \cdot\left(g_{\varepsilon} \mid f^{\prime}\right) .
\end{gathered}
$$

Proof. By the above arguments, one has

$$
\begin{gathered}
\lim _{z \rightarrow 0} \mathrm{I}_{z}(b)=\sum_{\varepsilon, \varepsilon(1) \in\{0,1\}}\left\langle D_{\varepsilon(1)}^{+} D_{\varepsilon}^{+} u, G(s)\right\rangle \\
\chi_{[S, T]}(s) \cdot\left(f \mid g_{\varepsilon}\right) \chi_{\left[S^{\prime}, T^{\prime}\right]}\left(g_{1-\varepsilon(1)} \mid f^{\prime}\right)\left(g_{1-\varepsilon} \mid g_{\varepsilon(1)}\right)_{-}+ \\
+\lim _{z \rightarrow 0} \sum_{\varepsilon, \varepsilon(1) \in\{0,1\}}\left\langle D_{\varepsilon(1)}^{+} D_{\varepsilon}^{+} u \otimes W\left(z \int_{S / z^{2}}^{T / z^{2}} S_{u} Q_{+} f d u\right) \Phi_{F} \otimes\right. \\
W\left(z^{2} \int_{S / z^{2}}^{T / z^{2}} Q^{-} S_{u} f d u\right) \Phi_{F}^{\iota},
\end{gathered}
$$




$$
\begin{aligned}
& \frac{1}{z} \chi_{[S, T]}(s) \cdot\left(f \mid g_{\varepsilon}\right)\left(g_{1-\varepsilon} \mid g_{\varepsilon(1)}\right)-\left[1 \otimes A\left(S_{s / z^{2}} Q+g_{1-\varepsilon(1)}\right) \otimes 1, U_{s / z^{2}}\right] \\
& \left.v \otimes W\left(z \int_{S^{\prime} / z^{2}}^{T^{\prime} / z^{2}} S_{u} Q_{+} f^{\prime} d u\right) \Phi_{F} \otimes W\left(z^{2} \int_{S^{\prime} / z^{2}}^{T^{\prime} / z^{2}} Q^{-} S_{u} f^{\prime} d u\right) \Phi_{F}^{\iota}\right\rangle \\
& =\sum_{\varepsilon, \varepsilon(1) \in\{0,1\}}\left\langle D_{\varepsilon(1)}^{+} D_{\varepsilon}^{+} u, G(s)\right\rangle \chi_{[s, T]}(s) \cdot\left(f \mid g_{\varepsilon}\right)\left(g_{1-\varepsilon} \mid g_{\varepsilon(1)}\right)-\chi_{\left[S^{\prime}, T^{\prime}\right]}(s)\left(g_{1-\varepsilon(1)} \mid f^{\prime}\right)+ \\
& +\sum_{\varepsilon, \varepsilon(1), \varepsilon(2) \in\{0,1\}}\left\langle D_{\varepsilon(2)}^{+} D_{\varepsilon(1)}^{+} D_{\varepsilon}^{+} u, G(s)\right\rangle\left(g_{1-\varepsilon} \mid g_{\varepsilon(1)}\right)_{-}\left(g_{1-\varepsilon(1)} \mid g_{\varepsilon(2)}\right)_{-} \\
& \chi_{[S, T]}(s) \cdot\left(f \mid g_{\varepsilon}\right) \chi_{\left[S^{\prime}, T^{\prime}\right]}(s)\left(g_{1-\varepsilon(2)} \mid f^{\prime}\right)+ \\
& +\lim _{z \rightarrow 0} \sum_{\varepsilon, \varepsilon(1), \varepsilon(2) \in\{0,1\}} \\
& \left\langle D_{\varepsilon(2)}^{+} D_{\varepsilon(1)}^{+} D_{\varepsilon}^{+} u \otimes W\left(z \int_{S / z^{2}}^{T / z^{2}} S_{u} Q_{+} f d u\right) \Phi_{F} \otimes W\left(z^{2} \int_{S / z^{2}}^{T / z^{2}} Q^{-} S_{u} f d u\right) \Phi_{F}^{\iota},\right. \\
& \chi_{[S, T]}(s) \cdot\left(f \mid g_{\varepsilon}\right)\left(g_{1-\varepsilon} \mid g_{\varepsilon(1)}\right)_{-} \cdot\left(g_{1-\varepsilon(1)} \mid g_{\varepsilon(2)}\right)_{-} \\
& \frac{1}{z}\left[1 \otimes A\left(S_{S / z^{2}} Q+g_{1-\varepsilon(2)}\right) \otimes 1, U_{\varepsilon / z^{2}}\right] \\
& \left.v \otimes W\left(z \int_{S^{\prime} / z^{2}}^{T^{\prime} / z^{2}} S_{u} Q_{+} f^{\prime} d u\right) \Phi_{F} \otimes W\left(z^{2} \int_{S^{\prime} / z^{2}}^{T^{\prime} / z^{2}} Q^{-} S_{u} f^{\prime} d u\right) \Phi_{F}^{c}\right\rangle
\end{aligned}
$$

Iterating, we obtain that

$$
\begin{aligned}
& \lim _{z \rightarrow 0} \mathrm{I}_{z}(b)=\sum_{\varepsilon, \varepsilon(1) \in\{0,1\}}\left\langle D_{\varepsilon(1)}^{+} D_{\varepsilon}^{+} u, G(s)\right\rangle \\
& \chi_{[S, T]}(s) \cdot\left(f \mid g_{\varepsilon}\right)\left(g_{1-\varepsilon} \mid g_{\varepsilon(1)}\right)-\chi_{\left[S^{\prime}, T^{\prime}\right]}(s)\left(g_{1-\varepsilon(1)} \mid f^{\prime}\right)+ \\
& +\sum_{\varepsilon, \varepsilon(1), \varepsilon(2) \in\{0,1\}}\left\langle D_{\varepsilon^{(2)}}^{+} D_{\varepsilon(1)}^{+} D_{\varepsilon}^{+} u, G(s)\right\rangle\left(g_{1-\varepsilon} \mid g_{\varepsilon(1)}\right)_{-}\left(g_{1-\varepsilon(1)} \mid g_{\varepsilon(2)}\right)_{-} \\
& \left.\chi_{[S, T]}(s) \cdot\left(f \mid g_{\varepsilon}\right) \chi_{\left[S^{\prime}, T^{\prime}\right]}(s)\left(g_{1-\varepsilon(2)}\right) \mid f^{\prime}\right)+ \\
& +\sum_{\varepsilon, \varepsilon(1), \varepsilon(2), \varepsilon(3) \in\{0,1\}}\left\langle D_{\varepsilon(3)}^{+} D_{\varepsilon(2)}^{+} D_{\varepsilon(1)}^{+} D_{\varepsilon}^{+} u, G(s)\right\rangle \\
& \left(g_{1-\varepsilon} \mid g_{\varepsilon(1)}\right)_{-} \cdot\left(g_{1-\varepsilon(1)} \mid g_{\varepsilon(2)}\right)_{-} \cdot\left(g_{1-\varepsilon(2)} \mid g_{\varepsilon(3)}\right)_{-} \\
& \left.\chi_{[S, T]}(s) \cdot\left(f \mid g_{\varepsilon}\right) \cdot \chi_{\left[S^{\prime}, T^{\prime}\right]}(s)\left(g_{1-\varepsilon(3)}\right) \mid f^{\prime}\right)+ \\
& +\cdots \cdots+ \\
& =\sum_{n=1}^{\infty} \sum_{\varepsilon, \varepsilon(1), \cdots, \varepsilon(n) \in\{0,1\}}\left\langle D_{\varepsilon(n)}^{+} \cdots \cdots \cdot D_{\varepsilon(1)}^{+} D_{\varepsilon}^{+} u, G(s)\right\rangle \\
& \left(g_{1-\varepsilon} \mid g_{\varepsilon(1)}\right)_{-} \cdot\left(g_{1-\varepsilon(1)} \mid g_{\varepsilon(2)}\right)_{-} \cdots \cdot\left(g_{1-\varepsilon(n-1)} \mid g_{\varepsilon(n)}\right)_{-}
\end{aligned}
$$


where, $\varepsilon(0):=\varepsilon$.

$$
\chi_{[S, T]}(s) \cdot\left(f \mid g_{\varepsilon}\right) \cdot \chi_{\left[S^{\prime}, T^{\prime}\right]}(s)\left(g_{1-\varepsilon(n)} \mid f^{\prime}\right)
$$

We split the right hand side of (6.43) into two terms according to

$$
\varepsilon(n)=\varepsilon
$$

or

$$
\varepsilon(n)=1-\varepsilon
$$

then the right hand side of (6.43) is equal to

$$
\begin{aligned}
& \sum_{n=1}^{\infty} \sum_{\varepsilon, \varepsilon(1), \cdots, \varepsilon(n-1) \in\{0,1\}}\left\langle D_{\varepsilon}^{+} D_{\varepsilon(n-1)}^{+} \cdots \cdots D_{\varepsilon(1)}^{+} D_{\varepsilon}^{+} u, G(s)\right\rangle \\
& \left(g_{1-\varepsilon} \mid g_{\varepsilon(1)}\right)_{-} \cdot\left(g_{1-\varepsilon(1)} \mid g_{\varepsilon(2)}\right)_{-} \cdots \cdot\left(g_{1-\varepsilon(n-1)} \mid g_{\varepsilon}\right)_{-} \\
& \chi_{[S, T]}(s) \cdot\left(f \mid g_{\varepsilon}\right) \chi_{\left[S^{\prime}, T^{\prime}\right]}(s) \cdot\left(g_{1-\varepsilon} \mid f^{\prime}\right) \\
& +\sum_{n=1}^{\infty} \sum_{\varepsilon, \varepsilon(1), \cdots, \varepsilon(n-1) \in\{0,1\}}\left\langle D_{1-\varepsilon}^{+} D_{\varepsilon(n-1)}^{+} \cdots \cdots D_{\varepsilon(1)}^{+} D_{\varepsilon}^{+} u, G(s)\right\rangle \\
& \left(g_{1-\varepsilon} \mid g_{\varepsilon(1)}\right)_{-} \cdot\left(g_{1-\varepsilon(1)} \mid g_{\varepsilon(2)}\right)_{-} \cdots \cdot\left(g_{1-\varepsilon(n-1)} \mid g_{1-\varepsilon)}\right)_{-} \\
& \chi_{[S, T]}(s) \cdot\left(f \mid g_{\varepsilon}\right) \cdot \chi_{\left[S^{\prime}, T^{\prime}\right]}(s) \cdot\left(g_{1-\varepsilon} \mid f^{\prime}\right) \\
& =\sum_{\varepsilon \in\{1,0\}} \sum_{n=0}^{\infty} \sum_{\sigma \in\{0,1\}^{n}}\left\langle D_{\varepsilon}^{+} D_{\sigma(n)}^{+} \cdots \cdots D_{\sigma(1)}^{+} D_{\varepsilon}^{+} u, G(s)\right\rangle \\
& \left(g_{1-\varepsilon} \mid g_{\varepsilon(1)}\right)_{-} \cdot\left(g_{1-\sigma(1)} \mid g_{\sigma(2)}\right)_{-} \cdots \cdot\left(g_{1-\sigma(n)} \mid g_{\varepsilon)}\right)_{-} \\
& \chi_{[S, T]}(s) \cdot\left(f \mid g_{\varepsilon}\right) \cdot \chi_{\left[S^{\prime}, T^{\prime}\right]}(s) \cdot\left(g_{1-\varepsilon} \mid f^{\prime}\right) \\
& +\sum_{\varepsilon \in\{1,0\}} \sum_{n=1}^{\infty} \sum_{\sigma \in\{0,1\} n}\left\langle D_{1-\varepsilon}^{+} D_{\sigma(n)}^{+} \cdots \cdots D_{\sigma(1)}^{+} D_{\varepsilon}^{+} u, G(s)\right\rangle \\
& \left(g_{1-\varepsilon} \mid g_{\sigma(1)}\right)_{-} \cdot\left(g_{1-\sigma(1)} \mid g_{\sigma(2)}\right)_{-} \cdots \cdot\left(g_{1-\sigma(n)} \mid g_{1-\varepsilon)}\right)_{-} \\
& \left(\chi_{[S, T]}(s) \cdot\left(f \mid g_{\varepsilon}\right) \cdot \chi_{\left[S^{\prime}, T^{\prime}\right]}(s) \cdot\left(g_{\varepsilon} \mid f^{\prime}\right)\right.
\end{aligned}
$$

where, $\sigma(0):=\varepsilon$. This finish the proof of (6.42).

Lemma (6.5).

$$
\lim _{z \rightarrow 0} \frac{1}{z} \mathrm{II}_{z}=0
$$

Proof.

$$
\frac{1}{z} \mathrm{II}_{z}=\sum_{\varepsilon \in\{0,1\}} \frac{1}{z}\left\langle D_{\varepsilon}^{+} u \otimes W\left(z \int_{S / z^{2}}^{T / z^{2}} S_{u} Q_{+} f d u\right) \Phi_{F} \otimes W\left(z^{2} \int_{S / z^{2}}^{T / z^{2}} Q-S_{u} f d u\right) \Phi_{F}^{\iota},\right.
$$


$1 \otimes A^{+}\left(S_{s / z^{2}} Q+g_{\varepsilon}\right) \otimes A^{+}\left(Q-S_{s / z^{2}} g_{1-\varepsilon}\right) U_{s / z^{2}}$

$$
\begin{aligned}
& \left.v \otimes W\left(z \int_{S^{\prime} / z^{2}}^{T^{\prime} / z^{2}} S_{u} Q_{+} f^{\prime} d u\right) \Phi_{F} \otimes W\left(z^{2} \int_{S^{\prime} / z^{2}}^{T^{\prime} / z^{2}} Q^{-} S_{u} f^{\prime} d u\right) \Phi_{F}^{\iota}\right\rangle \\
= & \left\langle D_{\varepsilon}^{+} u \otimes W\left(z \int_{S / z^{2}}^{T / z^{2}} S_{u} Q+f d u\right) \Phi_{F} \otimes W\left(z^{2} \int_{S / z^{2}}^{T / z^{2}} Q^{-} S_{u} f d u\right) \Phi_{F}^{\iota},\right.
\end{aligned}
$$

$$
\begin{gathered}
\sum_{\varepsilon \in\{0,1\}} z^{2} \int_{S / z^{2}}^{T / z^{2}}\left\langle S_{u} Q+f, S_{S / z^{2}} Q+g_{\varepsilon}\right\rangle d u \\
\int_{S / z^{2}}^{T / z^{2}}\left\langle Q^{-} S_{u} f, Q^{-} S_{S / z^{2}} g_{1-\varepsilon}\right\rangle_{\iota} d u \cdot U_{S / z^{2}}
\end{gathered}
$$

$\left.v \otimes W\left(z \int_{S^{\prime} / z^{2}}^{T^{\prime} / z^{2}} S_{u} Q_{+} f^{\prime} d u\right) \Phi_{F} \otimes W\left(z^{2} \int_{S^{\prime} / z^{2}}^{T^{\prime} / z^{2}} Q-S_{u} f^{\prime} d u\right) \Phi_{F}^{\iota}\right\rangle$

$$
=z^{2} \cdot O(1) \longrightarrow 0 \text { a.e. }
$$

LEMMA (6.6).

$$
\lim _{z \rightarrow 0} \mathrm{IV}_{z}=\sum_{\varepsilon \in\{0,1\}}\left\langle D_{\varepsilon}^{+} u, G(s)\right\rangle\left\langle g_{1-\varepsilon,} e^{-\frac{1}{2} \beta H} g_{\varepsilon}\right\rangle .
$$

Proof. It is enough to prove that the quantity

$$
\begin{gathered}
\operatorname{IV}(z, s):=\mid\left\langle D_{\varepsilon}^{+} u \otimes W\left(z \int_{S / z^{2}}^{T / z^{2}} S_{u} Q_{+} f d u\right) \Phi_{F} \otimes W\left(z^{2} \int_{S / z^{2}}^{T / z^{2}} S_{u} Q^{-} f d u\right) \Phi_{F}^{\iota},\right. \\
1 \otimes 1 \otimes A^{+}\left(S_{S / z^{2}} Q^{-} g_{1-\varepsilon}\right) A\left(S_{S / z^{2}} Q^{-} g_{\varepsilon}\right) U_{s / z^{2}} \\
\left.v \otimes W\left(z \int_{S^{\prime} / z^{2}}^{T^{\prime} / z^{2}} S_{u} Q_{+} f^{\prime} d u\right) \Phi_{F} \otimes W\left(z^{2} \int_{S^{\prime} / z^{2}}^{T^{\prime} / z^{2}} S_{u} Q^{-} f^{\prime} d u\right) \Phi_{F}^{\iota}\right\rangle \mid
\end{gathered}
$$

tends to zero (a.e.) as $z \rightarrow 0$.

Since the integral

$$
\int_{0}^{t} d s \operatorname{IV}(z, s)
$$

is a term of type II in the sense of Section $3\left(k^{\prime \prime}=0\right)$, one gets that

$$
\lim _{z \rightarrow 0} \int_{0}^{t} d s \operatorname{IV}(z, s)=0
$$

By the uniform estimate, we obtain

$$
0=\int_{0}^{t} d s \lim _{z \rightarrow 0} \operatorname{IV}(z, s)
$$


This result and the positivity of $\operatorname{IV}(\boldsymbol{z}, \boldsymbol{s})$ concludes the proof.

Our next step is to investigate the limit

$$
\lim _{z \rightarrow 0} \frac{1}{z} \mathrm{III}_{z}
$$

By definition

$$
\begin{gathered}
\frac{1}{z} \mathrm{III}_{z}=\frac{1}{z} \sum_{\varepsilon \in\{0,1\}}\left\langle D_{\varepsilon}^{+} u \otimes W\left(z \int_{S / z^{2}}^{T / z^{2}} S_{u} Q_{+} f d u\right) \Phi_{F} \otimes W\left(z^{2} \int_{S / z^{2}}^{T / z^{2}} Q^{-} S_{u} f d u\right) \Phi_{F}^{\iota},\right. \\
1 \otimes A\left(S_{s / z^{2}} Q_{+} g_{1-\varepsilon}\right) \otimes A\left(Q^{-} S_{s / z^{2}} g_{\varepsilon}\right) \cdot U_{s / z^{2}} \\
\left.v \otimes W\left(z \int_{S^{\prime} / z^{2}}^{T^{\prime} / z^{2}} S_{u} Q_{+} f^{\prime} d u\right) \Phi_{F} \otimes W\left(z^{2} \int_{S^{\prime} / z^{2}}^{T^{\prime} / z^{2}} Q^{-} S_{u} f^{\prime} d u\right) \Phi_{F}^{\iota}\right\rangle
\end{gathered}
$$

where, one can write the product

$$
1 \otimes A\left(S_{s / z^{2}} Q+g_{1-\varepsilon}\right) \otimes A\left(Q^{-} S_{s / z^{2}} g_{\varepsilon}\right) \cdot U_{s / z^{2}}
$$

to a sum of four terms:

$$
\begin{gathered}
U_{s / z^{2}} 1 \otimes A\left(S_{s / z^{2}} Q+g_{1-\varepsilon}\right) \otimes A\left(Q^{-} S_{s / z^{2}} g_{\varepsilon}\right)+ \\
+\left[1 \otimes A\left(S_{s / z^{2}} Q+g_{1-\varepsilon}\right) \otimes 1, U_{s / z^{2}}\right] 1 \otimes 1 \otimes A\left(Q^{-} S_{s / z^{2}} g_{\varepsilon}\right)+ \\
+\left[1 \otimes 1 \otimes A\left(Q^{-} S_{s / z^{2}} g_{\varepsilon}\right), U_{s / z^{2}}\right] 1 \otimes A\left(S_{s / z^{2}} Q_{+} g_{1-\varepsilon}\right) \otimes 1+ \\
+\left[1 \otimes A\left(S_{s / z^{2}} Q+g_{1-\varepsilon}\right) \otimes A\left(Q^{-} S_{s / z^{2}} g_{\varepsilon}\right) \cdot U_{s / z^{2}}\right]
\end{gathered}
$$

Respectively, $\frac{1}{z} \mathrm{III}_{z}$ can be written as a sum of four terms, namely

$$
\mathrm{III}_{z}(\mathrm{a})+\mathrm{III}_{z}(\mathrm{~b})+\mathrm{III}_{z}(\mathrm{c})+\mathrm{III}_{z}(\mathrm{~b})
$$

where,

$$
\begin{gathered}
\operatorname{III}_{z}(\mathrm{a}):=\frac{1}{z} \sum_{\varepsilon \in\{0,1\}}\left\langle D_{\varepsilon}^{+} u \otimes W\left(z \int_{S / z^{2}}^{T / z^{2}} S_{u} Q+f d u\right) \Phi_{F} \otimes\right. \\
W\left(z^{2} \int_{S / z^{2}}^{T / z^{2}} Q-S_{u} f d u\right) \Phi_{F}^{\iota}, \\
U_{s / z^{2}} 1 \otimes A\left(S_{S / z^{2}} Q+g_{1-\varepsilon}\right) \otimes A\left(Q-S_{S / z^{2}} g_{\varepsilon}\right) \\
\left.\otimes W\left(z \int_{S^{\prime} / z^{2}}^{T^{\prime} / z^{2}} S_{u} Q+f^{\prime} d u\right) \Phi_{F} \otimes W\left(z^{2} \int_{S^{\prime} / z^{2}}^{T^{\prime} / z^{2}} Q-S_{u} f^{\prime} d u\right) \Phi_{F}^{<}\right\rangle
\end{gathered}
$$


(6.57b)

$$
\begin{gathered}
\mathrm{III}_{z}(\mathrm{~b}):=\frac{1}{z} \sum_{\varepsilon \in\{0,1\}}\left\langle D_{\varepsilon}^{+} u \otimes W\left(z \int_{S / z^{2}}^{T / z^{2}} S_{u} Q_{+} f d u\right) \Phi_{F} \otimes W\left(z^{2} \int_{S / z^{2}}^{T / z^{2}} Q^{-} S_{u} f d u\right) \Phi_{F}^{\iota},\right. \\
{\left[1 \otimes A\left(S_{S / z^{2}} Q_{+} g_{1-\varepsilon}\right) \otimes 1, U_{s / z^{2}}\right] 1 \otimes 1 \otimes A\left(Q^{-} S_{S / z^{2}} g_{\varepsilon}\right)} \\
\left.v \otimes W\left(z \int_{S^{\prime} / z^{2}}^{T^{\prime} / z^{2}} S_{u} Q_{+} f^{\prime} d u\right) \Phi_{F} \otimes W\left(z^{2} \int_{S^{\prime} / z^{2}}^{T^{\prime} / z^{2}} Q^{-} S_{u} f^{\prime} d u\right) \Phi_{F}^{\iota}\right\rangle
\end{gathered}
$$

$\operatorname{III}_{z}(\mathrm{c}):=\frac{1}{z} \sum_{\varepsilon \in\{0,1\}}\left\langle D_{\varepsilon}^{+} u \otimes W\left(z \int_{S / z^{2}}^{T / z^{2}} S_{u} Q_{+} f d u\right) \Phi_{F} \otimes W\left(z^{2} \int_{S / z^{2}}^{T / z^{2}} Q^{-} S_{u} f d u\right) \Phi_{F}^{\iota}\right.$,

$\left[1 \otimes 1 \otimes A\left(Q^{-} S_{s / z^{2}} g_{1-\varepsilon}\right), U_{s / z^{2}}\right] 1 \otimes A\left(S_{s / z^{2}} Q+g_{1-\varepsilon}\right) \otimes 1$

$$
\left.v \otimes W\left(z \int_{S^{\prime} / z^{2}}^{T^{\prime} / z^{2}} S_{u} Q+f^{\prime} d u\right) \Phi_{F} \otimes W\left(z^{2} \int_{S^{\prime} / z^{2}}^{T^{\prime} / z^{2}} Q^{-} S_{u} f^{\prime} d u\right) \Phi_{F}^{\iota}\right\rangle
$$

and

$$
\begin{gathered}
\operatorname{III}_{z}(\mathrm{~d}):=\frac{1}{z} \sum_{\varepsilon \in\{0,1\}}\left\langle D_{\varepsilon}^{+} u \otimes W\left(z \int_{S / z^{2}}^{T / z^{2}} S_{u} Q_{+} f d u\right) \Phi_{F} \otimes\right. \\
W\left(z^{2} \int_{S / z^{2}}^{T / z^{2}} Q^{-} S_{u} f d u\right) \Phi_{F}^{\iota} \\
{\left[1 \otimes A\left(S_{S / z^{2}} Q+g_{1-\varepsilon}\right) \otimes A\left(Q^{-} S_{S / z^{2}} g_{\varepsilon}\right) \cdot U_{s / z^{2}}\right]} \\
\left.v \otimes W\left(z \int_{S^{\prime} / z^{2}}^{T^{\prime} / z^{2}} S_{u} Q_{+} f^{\prime} d u\right) \Phi_{F} \otimes W\left(z^{2} \int_{S^{\prime} / z^{2}}^{T^{\prime} / z^{2}} Q^{-} S_{u} f^{\prime} d u\right) \Phi_{F}^{\iota}\right\rangle .
\end{gathered}
$$

For the four terms we have that

LEMMa (6.7).

$$
\operatorname{III}_{z}(a)=z^{2} \cdot O(1) \longrightarrow 0
$$

Proof. In the right hand side of (6.57a), applying the two annihilation operators on the coherent vectors and we obtain that

$$
\begin{gathered}
\operatorname{III}_{z}(\mathrm{a})=\frac{1}{z} \sum_{\varepsilon \in\{0,1\}}\left\langle D_{\varepsilon}^{+} u \otimes W\left(z \int_{S / z^{2}}^{T / z^{2}} S_{u} Q+f d u\right) \Phi_{F} \otimes W\left(z^{2} \int_{S / z^{2}}^{T / z^{2}} Q-S_{u} f d u\right) \Phi_{F}^{\epsilon}\right. \\
U_{S / z^{2}} z \int_{S^{\prime} / z^{2}}^{T^{\prime} / z^{2}}\left\langle S_{S / z^{2}} Q+g_{1-\varepsilon}, S u Q+f^{\prime}\right\rangle d u \\
z^{2} \int_{S^{\prime} / z^{2}}^{T^{\prime} / z^{2}}\left\langle Q^{-} S_{S / z^{2}} g_{\varepsilon}, Q-S u f^{\prime}\right\rangle, d u
\end{gathered}
$$




$$
\begin{gathered}
\left.v \otimes W\left(z \int_{S^{\prime} / z^{2}}^{T^{\prime} / z^{2}} S_{u} Q+f^{\prime} d u\right) \Phi_{F} \otimes W\left(z^{2} \int_{S^{\prime} / z^{2}}^{T^{\prime} / z^{2}} Q-S_{u} f^{\prime} d u\right) \Phi_{F}^{<}\right\rangle \\
=z^{2} \cdot \sum_{\varepsilon \in\{0,1\}} \int_{S^{\prime} / z^{2}}^{T^{\prime} / z^{2}}\left\langle S_{S / z^{2}} Q+g_{1-\varepsilon}, S_{u} Q+f^{\prime}\right\rangle d u . \\
\cdot \int_{S^{\prime} / z^{2}}^{T^{\prime} / z^{2}}\left\langle Q^{-} S_{S / z^{2}} g_{\varepsilon}, Q-S u f^{\prime}\right\rangle, d u \\
\left\langle D_{\varepsilon}^{+} u, G_{z}(s)\right\rangle .
\end{gathered}
$$

This gives (6.58).

Lemma (6.8).

$$
\mathrm{III}_{z}(\mathrm{~b})=z^{2} \cdot O(1) \longrightarrow 0 .
$$

Proof. In the right hand side of $(6.57 \mathrm{~b})$, apply the annihilation operator $A\left(Q^{-} S_{S / z^{2}} g_{\varepsilon}\right)$ on the coherent vector

$$
W\left(z^{2} \int_{S^{\prime} / z^{2}}^{T^{\prime} / z^{2}} Q^{-} S_{u} f^{\prime} d u\right) \Phi_{F}^{\iota}
$$

one finds that

$$
\begin{gathered}
\operatorname{III}_{z}(\mathrm{~b})=\sum_{\varepsilon \in\{0,1\}} z^{2} \int_{S / z^{2}}^{T / z^{2}}\left\langle Q^{-} S_{S / z^{2}} g_{\varepsilon}, Q^{-} S_{u} f^{\prime}\right\rangle_{\iota} d u \\
\left\langle D_{\varepsilon}^{+} u \otimes W\left(z \int_{S / z^{2}}^{T / z^{2}} S_{u} Q+f d u\right) \Phi_{F} \otimes W\left(z^{2} \int_{S / z^{2}}^{T / z^{2}} Q^{-} S_{u} f d u\right) \Phi_{F}^{\iota},\right. \\
\frac{1}{z}\left[1 \otimes A\left(S_{S / z^{2}} Q+g_{1-\varepsilon}\right) \otimes 1, U_{S / z^{2}}\right] \\
\left.v \otimes W\left(z \int_{S^{\prime} / z^{2}}^{T^{\prime} / z^{2}} S_{u} Q_{+} f^{\prime} d u\right) \Phi_{F} \otimes W\left(z^{2} \int_{S^{\prime} / z^{2}}^{T^{\prime} / z^{2}} Q^{-} S_{u} f^{\prime} d u\right) \Phi_{F}^{\iota}\right\rangle .
\end{gathered}
$$

This formula and Lemma (6.2) imply (6.60).

LEMma (6.9).

$$
\mathrm{III}_{z}(\mathrm{c}) \longrightarrow 0
$$

Proof. This Lemma is a direct result of Lemma (6.3).

Our next goal is to obtain the limit of $\operatorname{III}_{z}(\mathrm{~d})$

Lemma (6.10). For $g_{0}, g_{1}, t$ satisfying the condition ( $\left.*\right)$, the limit

$$
\lim _{z \rightarrow 0} \int_{0}^{t} \operatorname{III}_{z}(\mathrm{~d}) d s
$$


exists and is equal to

$$
\sum_{\varepsilon \in\{0,1\}} \int_{0}^{t} d t_{0}\left\langle u, D_{3}(\varepsilon) G\left(t_{0}\right)\right\rangle
$$

where,

$$
\begin{aligned}
& D_{3}(\varepsilon):=\sum_{k=1}^{\infty} \sum_{\sigma \in\{0,1\} k} D_{\varepsilon} D_{\sigma(k)} \cdots D_{\sigma(1)} \int_{-\infty}^{0} d s_{1} \cdots \int_{-\infty}^{0} d s_{k}\left\langle g_{1-\varepsilon}, S_{s_{1}} g_{\sigma(1)}\right\rangle \\
& \left\langle g_{1-\sigma(1)}, S_{s 2} g_{\sigma(2)}\right\rangle \cdots\left\langle g_{1-\sigma(k-1)}, S_{s k} g_{1-\sigma(k)}\right\rangle \cdot \overline{\left\langle g_{\varepsilon}, S_{s_{k}+\cdots+s 1} g_{\sigma(k)}\right\rangle}
\end{aligned}
$$

Proof. With change of variable

$$
s / z^{2}=t_{0}
$$

one gets

$$
\int_{0}^{t} \operatorname{III}_{z}(\mathrm{~d}) d s=\frac{1}{z} \sum_{\varepsilon \in\{0,1\}}
$$

$$
\begin{gathered}
\left\langle D_{\varepsilon}^{+} u \otimes W\left(z \int_{S / z^{2}}^{T / z^{2}} S_{u} Q_{+} f d u\right) \Phi_{F} \otimes W\left(z^{2} \int_{S / z^{2}}^{T / z^{2}} Q-S_{u} f d u\right) \Phi_{F}^{\iota},\right. \\
\int_{0}^{t} d s\left[1 \otimes A\left(S_{S / z^{2}} Q_{+} g_{1-\varepsilon}\right) \otimes A\left(Q^{-} S_{S / z^{2}} g_{\varepsilon}\right), U_{S / z^{2}}\right]
\end{gathered}
$$

$\left.v \otimes W\left(z \int_{S^{\prime} / z^{2}}^{T^{\prime} / z^{2}} S_{u} Q_{+} f^{\prime} d u\right) \Phi_{F} \otimes W\left(z^{2} \int_{S^{\prime} / z^{2}}^{T^{\prime} / z^{2}} Q^{-} S_{u} f^{\prime} d u\right) \Phi_{F}^{\iota}\right\rangle$

$=\sum_{\varepsilon \in\{0,1\}}\left\langle D_{\varepsilon}^{+} u \otimes W\left(z \int_{S / Z^{2}}^{T / z^{2}} S_{u} Q+f d u\right) \Phi_{F} \otimes W\left(z_{2} \int_{S / z^{2}}^{T / z^{2}} Q^{-} S_{u} f d u\right) \Phi_{F}^{\epsilon}\right.$,

$$
\int_{0}^{t / z^{2}} d t_{0} z\left[1 \otimes A\left(S_{t 0} Q+g_{1-\varepsilon}\right) \otimes A\left(Q^{-} S_{t 0} g_{\varepsilon}\right), U_{t 0}\right]
$$

$\left.v \otimes W\left(z \int_{S^{\prime} / z^{2}}^{T^{\prime} / z^{2}} S_{u} Q_{+} f^{\prime} d u\right) \Phi_{F} \otimes W\left(z^{2} \int_{S^{\prime} / z^{2}}^{T^{\prime} / z^{2}} Q^{-} S_{u} f^{\prime} d u\right) \Phi_{F}^{\iota}\right\rangle$

$=\sum_{\varepsilon \in\{0,1\}} \sum_{n=0}^{\infty}\left\langle D_{\varepsilon}^{+} u \otimes W\left(z \int_{S / Z^{2}}^{T / z^{2}} S_{u} Q_{+} f d u\right) \Phi_{F} \otimes W\left(z \int_{S / z^{2}}^{T / z^{2}} Q^{-} S_{u} f d u\right) \Phi_{F}^{\iota}\right.$,

$$
(-i)^{n} z \int_{0}^{t / z^{2}} d t_{0} \int_{0}^{t_{0}} d t_{1} \cdots \int_{0}^{t_{n-1}} d t_{n}
$$

$$
\times\left[1 \otimes A\left(S_{t_{0}} Q+g_{1-\varepsilon}\right) \otimes A\left(Q-S_{t_{0}} g_{\varepsilon}\right), V\left(t_{1}\right) \cdots V\left(t_{n}\right)\right]
$$

$\left.v \otimes W\left(z \int_{S^{\prime} / z^{2}}^{T^{\prime} / z^{2}} S_{u} Q_{+} f^{\prime} d u\right) \Phi_{F} \otimes W\left(z^{2} \int_{S^{\prime} / z^{2}}^{T^{\prime} / z^{2}} Q^{-} S_{u} f^{\prime} d u\right) \Phi_{F}^{\iota}\right\rangle$.

Thus by the uniform estimate, we can exchange the order of the limit of $z \rightarrow 0$ and the sum over $n \in \mathbf{N}$.

Notice that for any operators $A, B, X$, 
(6.68)

$$
A B=B A \Longrightarrow[A B, X]=[A,[B, X]]
$$

therefore

$$
\begin{gathered}
{\left[1 \otimes A\left(S_{t 0} Q+g_{1-\varepsilon}\right) \otimes A\left[Q^{-} S_{t 0} g_{\varepsilon}\right), V\left(t_{1}\right) \cdots V\left(t_{n}\right)\right]} \\
=\left[1 \otimes A\left(S_{t_{0}} Q+g_{1-\varepsilon}\right) \otimes 1,\left[1 \otimes 1 \otimes A\left[Q^{-} S_{t o} g_{\varepsilon}\right), V\left(t_{1}\right) \cdots V\left(t_{n}\right)\right]\right] \\
=\sum_{k=1}^{n}\left[1 \otimes A\left(S_{t_{0}} Q+g_{1-\varepsilon}\right) \otimes 1, V\left(t_{1}\right) \cdots V\left(t_{k-1}\right)\right. \\
\left.\left[1 \otimes 1 \otimes A\left(Q^{-} S_{t_{0}} g_{\varepsilon}\right), V\left(t_{k}\right)\right] V\left(t_{k+1}\right) \cdots V\left(t_{n}\right)\right] \\
=\sum_{k=1}^{n} \sum_{\varepsilon(k) \in\{0,1\}}\left[1 \otimes A\left(S_{t_{0}} Q+g_{1-\varepsilon}\right) \otimes 1, V\left(t_{1}\right) \cdots V\left(t_{k-1}\right)\right. \\
{\left[1 \otimes 1 \otimes A\left(Q^{-} S_{t 0} g_{\varepsilon}\right), i\left(z A^{+}\left(S_{t k} Q+g_{1-\varepsilon(k)}\right) \otimes A^{+}\left(Q^{-} S_{t k} g_{\varepsilon(k)}\right)\right.\right.} \\
\left.\left.\left.+z^{2} 1 \otimes 1 \otimes A^{+}\left(Q^{-} S_{t_{k}} g_{1-\varepsilon(k)}\right) A\left(Q^{-} S_{t_{k}} g_{\varepsilon(k)}\right)\right)\right] V\left(t_{k+1}\right) \cdots V\left(t_{n}\right)\right] .
\end{gathered}
$$

Respectively, the right hand side of (6.67) becomes

(6.70) $\sum_{\varepsilon \in\{0,1\}} \sum_{n=0}^{\infty}\left\langle D_{\varepsilon}^{+} u \otimes W\left(z \int_{S / z^{2}}^{T / z^{2}} S_{u} Q_{+} f d u\right) \Phi_{F} \otimes W\left(z_{2} \int_{S / z^{2}}^{T / z^{2}} Q-S_{u} f d u\right) \Phi_{F}^{\iota}\right.$,

$$
(-i)^{n-1} z \int_{0}^{t / z^{2}} d t_{0} \int_{0}^{t_{0}} d t_{1} \cdots \int_{0}^{t_{n-1}} d t_{n} \sum_{k=1}^{n} \sum_{\varepsilon(k) \in\{0,1\}}\left\langle Q-S_{t 0} g_{\varepsilon}, Q-S_{t k} g_{\varepsilon(h)}\right\rangle_{\iota}
$$

$\left[1 \otimes A\left(S_{t_{0}} Q_{+} g_{1-\varepsilon}\right) \otimes 1, V\left(t_{1}\right) \cdots V\left(t_{k-1}\right) z \cdot 1 \otimes A^{+}\left(S_{t_{k}} Q_{+} g_{1-\varepsilon(k)}\right) \otimes 1\right]$

$\left.V\left(t_{k-1}\right) \cdots V\left(t_{n}\right) v \otimes W\left(z \int_{S^{\prime} / z^{2}}^{T^{\prime} / z^{2}} S_{u} Q_{+} f^{\prime} d u\right) \Phi_{F} \otimes W\left(z^{2} \int_{S^{\prime} / z}^{T^{\prime} / z^{2}} Q-S_{u} f^{\prime} d u\right) \Phi_{F}^{\iota}\right\rangle$

$+\sum_{\varepsilon \in\{0,1\}} \sum_{n=0}^{\infty}\left\langle D_{\varepsilon}^{+} u \otimes W\left(z \int_{S / z^{2}}^{T / z^{2}} S_{u} Q_{+} f d u\right) \Phi_{F} \otimes W\left(z_{2} \int_{S / z^{2}}^{T / z^{2}} Q^{-} S_{u} f d u\right) \Phi_{F}^{\iota}\right.$

$$
\begin{gathered}
(-i)^{n-1} z \int_{0}^{t / z^{2}} d t_{0} \int_{0}^{t_{0}} d t_{1} \cdots \int_{0}^{t_{n-1}} d t_{n} \\
\sum_{k=1}^{n} \sum_{\varepsilon(k) \in\{0,1\}}\left\langle\mathrm{Q}-S_{t 0} g_{\varepsilon}, Q-S_{t k} g_{\varepsilon(h)}\right\rangle_{\iota} V\left(t_{1}\right) \cdots V\left(t_{k-1}\right) z \cdot 1 \otimes
\end{gathered}
$$

$\otimes A^{+}\left(S_{t_{k}} Q_{+} g_{1-\varepsilon(k)}\right) \otimes 1$

$$
\left[1 \otimes A\left(S_{t_{0}} Q+g_{1-\varepsilon}\right) \otimes 1, V\left(t_{k-1}\right) \cdots V\left(t_{n}\right)\right]
$$

$\left.v \otimes W\left(z \int_{S^{\prime} / z^{2}}^{T^{\prime} / z^{2}} S_{u} Q+f^{\prime} d u\right) \Phi_{F} \otimes W\left(z^{2} \int_{S^{\prime} / z^{2}}^{T^{\prime} / z^{2}} Q-S_{u} f^{\prime} d u\right) \Phi_{F}^{c}\right\rangle$ 


$$
\begin{gathered}
+\sum_{\varepsilon \in\{0,1\}} \sum_{n=0}^{\infty}\left\langle D_{\varepsilon}^{+} u \otimes W\left(z \int_{S / z^{2}}^{T / z^{2}} S_{u} Q+f d u\right) \Phi_{F} \otimes W\left(z^{2} \int_{S / z^{2}}^{T / z^{2}} Q-S_{u} f d u\right) \Phi_{F}^{\iota},\right. \\
(-i)^{n-1} z \int_{0}^{t / z^{2}} d t_{0} \int_{0}^{t_{0}} d t_{1} \cdots \int_{0}^{t_{n-1}} d t_{n} \sum_{k=1}^{n} \sum_{\varepsilon(k) \in\{0.1\}}\left[1 \otimes A\left(S_{t_{0}} Q+g_{1-\varepsilon}\right) \otimes 1\right. \\
V\left(t_{1}\right) \cdots V\left(t_{k-1}\right)
\end{gathered}
$$

$\left[1 \otimes 1 \otimes A\left(Q^{-} S_{t 0} g_{\varepsilon}\right), z^{2} 1 \otimes 1 \otimes A^{+}\left(Q^{-} S_{t k} g_{1-\varepsilon(k)}\right) A\left(Q^{-} S_{t k} g_{\varepsilon(k)}\right)\right]$

$$
\begin{gathered}
\left.\times V\left(t_{k+1}\right) \cdots V\left(t_{n}\right)\right] \\
\left.v \otimes W\left(z \int_{S^{\prime} / z^{2}}^{T^{\prime} / z^{2}} S_{u} Q_{+} f^{\prime} d u\right) \Phi_{F} \otimes W\left(z^{2} \int_{S^{\prime} / z^{2}}^{T^{\prime} / z^{2}} Q^{-} S_{u} f^{\prime} d u\right) \Phi_{F}^{\iota}\right\rangle .
\end{gathered}
$$

Moreover, the third term of (6.70) is a type II term in the sense explained in Section $3\left(k^{\prime \prime}>0\right)$, so it goes to zero as $z \rightarrow 0$. The second term of $(6.70)$ corresponds to the situation in which there exists some $p_{h}$ or $p_{h}^{\prime}$ (here, it is 0 ) such that $q_{h}>p_{h}+1$ or $q_{h}^{\prime}>p_{1}^{\prime}+1$.

Therefore this term tends to zero as $z \rightarrow 0$.

Now let us consider the first term of (6.70). In the language of Theorem (4.8), $t_{0}$ is some $t_{i}$ and $t_{k}$ is some $t_{i}$, therefore we know that one needs only to consider the situation

$$
i_{1}<i_{1}^{\prime}<i_{2}<i_{2}^{\prime}<\cdots<i_{k}<i_{k}^{\prime}
$$

and the limit of the first term as $z \rightarrow 0$ is equal to

$$
\begin{gathered}
\sum_{n=1}^{\infty} \sum_{\varepsilon \in\{0,1\}} \lim _{z \rightarrow 0}\left\langle D_{\varepsilon}^{+} u \otimes W\left(z \int_{S / z^{2}}^{T / z^{2}} S_{u} Q+f d u\right) \Phi_{F} \otimes W\left(z^{2} \int_{S / z^{2}}^{T / z^{2}} Q-S_{u} f d u\right) \Phi_{F}^{\iota},\right. \\
(-i)^{n-1} z \int_{0}^{t / z^{2}} d t_{0} \int_{0}^{t_{0}} d t_{1} \cdots \int_{0}^{t n-1} d t_{n} \sum_{k=1}^{n} \sum_{\sigma(k) \in\{0,1\} k}\left\langle Q^{-} S_{t 0} g_{\varepsilon}, Q^{-} S_{t k} g_{\sigma(k)}\right\rangle_{\iota} \\
z D_{\sigma(1)} \cdots D_{\sigma(k)} \otimes\left[A\left(S_{t 0} Q_{+} g_{1-\varepsilon}\right), A^{+}\left(S_{t 1} Q+g_{\sigma(1)}\right) A\left(S_{t_{1}} Q+g_{1-\sigma(1)}\right) \cdots\right. \\
\left.A^{+}\left(S_{t_{k-1}} Q+g_{\sigma(k-1)}\right) A\left(S_{t k-1} Q+g_{1-\sigma(k-1)}\right) A^{+}\left(S_{t_{k}} Q+g_{1-\sigma(k)}\right)\right] \otimes 1 V\left(t_{k-1}\right) \cdots V\left(t_{n}\right) \\
\left.v \otimes W\left(z \int_{S^{\prime} / z^{2}}^{T^{\prime} / z^{2}} S_{u} Q+f^{\prime} d u\right) \Phi_{F} \otimes W\left(z^{2} \int_{S^{\prime} / z^{2}}^{T^{\prime} / z^{2}} Q-S_{u} f^{\prime} d u\right) \Phi_{F}^{\iota}\right\rangle .
\end{gathered}
$$

By Theorem (4.8), we know that one needs only to consider the case in which the operator

$$
\left[A\left(S_{t_{0}} Q+g_{1-\varepsilon}\right), A+\left(S_{t_{1}} Q+g_{\sigma(1)}\right) A\left(S_{t_{1}} Q+g_{1-\sigma(1)}\right) \cdots\right.
$$




$$
\left.A^{+}\left(S_{t_{k-1}} Q+g_{\sigma(k-1)}\right) A\left(S_{t_{k-1}} Q+g_{1-\sigma(k-1)}\right) A^{+}\left(S_{t_{k}} Q-g_{1-\sigma(k)}\right)\right]
$$

is replaced by its pure scalar product term

$$
\begin{gathered}
\left\langle S_{t_{0}} Q+g_{1-\varepsilon}, S_{t_{1}} Q+g_{\sigma(1)}\right\rangle \cdot\left\langle S_{t_{1}} Q+g_{1-\sigma(1)}, S_{t_{2}} Q_{+} g_{\sigma(2)}\right\rangle \cdots \\
\cdots\left\langle S_{t_{k-1}} Q+g_{1-\sigma(k-1)}, S_{t_{k}} Q+g_{1-\sigma(k)}\right\rangle
\end{gathered}
$$

therefore one has

$$
\lim _{z \rightarrow 0} \int_{0}^{t} \mathrm{III}_{z}(\mathrm{~d}) d s
$$

$=\sum_{n=0}^{\infty} \sum_{\varepsilon \in\{0,1\}} \lim _{z \rightarrow 0}\left\langle D_{\varepsilon}^{+} u \otimes W\left(z \int_{S / z^{2}}^{T / z^{2}} S_{u} Q_{+} f d u\right) \Phi_{F} \otimes W\left(z^{2} \int_{S / z^{2}}^{T / z^{2}} Q^{-} S_{u} f d u\right) \Phi_{F}^{\iota}\right.$,

$$
(-i)^{n-1} z^{2} \int_{0}^{t / z^{2}} d t_{0} \int_{0}^{t_{0}} d t_{1} \cdots \int_{0}^{t_{n-1}} d t_{n} \sum_{k=1}^{n} \sum_{\varepsilon(k) \in\{0,1\} k}\left\langle Q-S_{t 0} g_{\varepsilon}, Q-S_{t k} g_{\sigma(h)}\right\rangle_{\iota}
$$

$$
\begin{aligned}
D_{\sigma(1)} & \cdots D_{\sigma(k)} \otimes 1\left\langle S_{t_{0}} Q_{+} g_{1-\varepsilon}, S_{t_{1}} Q_{+} g_{\sigma(1)}\right\rangle \cdot \\
& \cdot\left\langle S_{t_{1}} Q_{+} g_{1-\sigma(1)}, S_{t_{2}} Q_{+} g_{\sigma(2)}\right\rangle \cdots \\
\cdots & \left\langle S_{t_{k-1}} Q_{+} g_{1-\sigma(k-1)}, S_{t_{k}} Q_{+} g_{1-\sigma(k)}\right\rangle
\end{aligned}
$$

$\left.V\left(t_{k+1}\right) \cdots V\left(t_{n}\right) v \otimes W\left(z \int_{S^{\prime} / z^{2}}^{T^{\prime} / z^{2}} S_{u} Q_{+} f^{\prime} d u\right) \Phi_{F} \otimes W\left(z^{2} \int_{S^{\prime} / z^{2}}^{T^{\prime} / z^{2}} Q^{-} S_{u} f^{\prime} d u\right) \Phi_{F}^{\iota}\right\rangle$

In the right hand side of (6.74), changing the order of sum for $n=1,2, \ldots$ and $k=$ $1, \ldots, n$ and with the change of variables

$$
z^{2} t_{h} \hookrightarrow t_{h}, h=0,1, \ldots, k
$$

we obtain that

$$
\lim _{z \rightarrow 0} \int_{0}^{t} \operatorname{III}_{z}(\mathrm{~d}) d s=\sum_{k=1}^{\infty} \sum_{\varepsilon \in\{0,1\}} \sum_{\sigma \in\{0,1\} k} \lim _{z \rightarrow 0}
$$

$$
\begin{gathered}
\left\langle D_{\sigma(k)}^{+} \cdots D_{\sigma(1)}^{+} D_{\varepsilon}^{+} u \otimes W\left(z \int_{S / z^{2}}^{T / z^{2}} S_{u} Q+f d u\right) \Phi_{F} \otimes W\left(z^{2} \int_{S / z^{2}}^{T / z^{2}} Q^{-} S_{u} f d u\right) \Phi_{F}^{\iota},\right. \\
z^{-2 k} \int_{0}^{t} d t_{0} \int_{0}^{t_{0}} d t_{1} \cdots \int_{0}^{t_{k-1}} d t_{k}\left\langle S_{t_{0} / z^{2}} Q+g_{1-\varepsilon}, S_{t_{1} / z^{2}} Q+g_{\sigma(1)}\right\rangle \\
\left\langle S_{t_{1} / z^{2}} Q+g_{1-\sigma(1)}, S_{t_{2} / z^{2}} Q+g_{\sigma(2)}\right\rangle \cdots\left\langle S_{t_{k-1} / z^{2}} Q+g_{1-\sigma(k-1)}, S_{t_{k} / z^{2}} Q_{+} g_{1-\sigma(k)}\right\rangle \\
\left\langle Q^{-} S_{t_{0}} g_{\varepsilon}, Q^{-} S_{t_{k}} g_{\sigma(k)}\right\rangle_{\iota} \\
\sum_{n=k}^{\infty}(-i)^{n-k} \int_{0}^{t_{k} / z^{2}} d t_{k+1} \cdots \int_{0}^{t_{n-1}} d t_{n} V\left(t_{k+1}\right) \cdots V\left(t_{n}\right)
\end{gathered}
$$




$$
\left.v \otimes W\left(z \int_{S^{\prime} / z^{2}}^{T^{\prime} / z^{2}} S_{u} Q_{+} f^{\prime} d u\right) \Phi_{F} \otimes W\left(z^{2} \int_{S^{\prime} / z^{2}}^{T^{\prime} / z^{2}} Q^{-} S_{u} f^{\prime} d u\right) \Phi_{F}^{c}\right\rangle
$$

In the right hand side of (6.76), with the change of variables

$$
s_{h}:=\left(t_{h}-t_{h-1}\right) / z^{2}, h=1, \ldots, k
$$

and applying Lemma (6.3) of [2], we find that

$$
\begin{gathered}
\lim _{z \rightarrow 0} \int_{0}^{t} \operatorname{III}_{z}(\mathrm{~d}) d s=\sum_{k=1}^{\infty} \sum_{\varepsilon \in\{0,1\}} \sum_{\sigma \varepsilon \in\{0,1\}^{k}} \int_{0}^{t} d t_{0} \\
\int_{-\infty}^{0} d s_{1} \cdots \int_{-\infty}^{0} d s_{k}\left\langle g_{1-\varepsilon}, S_{s_{1}} Q+g_{\sigma(1)}\right\rangle \\
\left\langle g_{1-\sigma(1)}, S_{s_{2}} Q+g_{\sigma(2)}\right\rangle \cdots\left\langle g_{1-\sigma(k-1)}, S_{s_{k}} Q+g_{1-\sigma(k)}\right\rangle \cdot \overline{\left\langle g_{\varepsilon}, S_{s_{k}+\cdots+s_{1}} g_{\sigma(k)}\right\rangle} \\
\lim _{z \rightarrow 0}\left\langle D_{\sigma(k)}^{+} \cdots D_{\sigma(1)}^{+} D_{\varepsilon}^{+} u \otimes W\left(z \int_{S / z^{2}}^{T / z^{2}} S_{u} Q+f d u\right) \Phi_{F} \otimes W\left(z^{2} \int_{S / z^{2}}^{T / z^{2}} Q^{-} S_{u} f d u\right) \Phi_{F}^{\iota},\right. \\
\left.U_{t_{0} / z^{2}} v \otimes W\left(z \int_{S^{\prime} / z^{2}}^{T^{\prime} / z^{2}} S_{u} Q+f^{\prime} d u\right) \Phi_{F} \otimes W\left(z^{2} \int_{S^{\prime} / z^{2}}^{T^{\prime} / z^{2}} Q^{-} S_{u} f^{\prime} d u\right) \Phi_{F}^{\iota}\right\rangle \\
=\sum_{\varepsilon \in\{0,1\}} \sum_{k=1}^{\infty} \sum_{\sigma \in\{0,1\} k} \int_{0}^{t} d t_{0}\left\langle D_{\sigma(k)}^{+} \cdots D_{\sigma(1)}^{+} D_{\varepsilon}^{+} u, G\left(t_{0}\right)\right\rangle \\
\int_{-\infty}^{0} d s_{1} \cdots \int_{-\infty}^{0} d s_{k}\left\langle g_{1-\varepsilon}, S_{s 1} g_{\sigma(1)}\right\rangle \\
\left\langle g_{1-\sigma(1)}, S_{s_{2}} Q+g_{\sigma(2)}\right\rangle \cdots\left\langle g_{1-\sigma(k-1)}, S_{s_{k}} Q+g_{1-\sigma(k)}\right\rangle \cdot \overline{\left\langle g_{\varepsilon}, S_{s_{k}+\cdots+s_{1}} g_{\sigma(k)}\right\rangle}
\end{gathered}
$$

this ends the proof of (6.64).

Finally, combining together (6.7), (6.9), (6.11), (6.12), (6.13), Lemma (6.2)-Lemma (6.10), we get the following

TheOREM (6.11). For any $t, g_{0}, g_{1}$ satisfied the condition (1.15),

$$
\begin{gathered}
\langle u, G(t)\rangle=\langle u, G(0)\rangle+\sum_{\varepsilon\{0,1\}} \int_{0}^{t} d s \\
\left(\chi_{[s, T]}(s) \cdot \chi_{\left[S^{\prime}, T^{\prime}\right]}(s) \cdot\left(g_{\varepsilon} \mid f^{\prime}\right) \cdot\left(f \mid g_{\varepsilon}\right)\left\langle D_{1}^{+}(\varepsilon) u, G(s)\right\rangle+\right. \\
+\chi_{[s, T]}(s) \cdot \chi_{\left[S^{\prime}, T^{\prime}\right]}(s) \cdot\left(f \mid g_{\varepsilon}\right) \cdot\left(g_{1-\varepsilon} \mid f^{\prime}\right) \cdot\left\langle D_{2}^{+}(\varepsilon) u, G(s)\right\rangle+ \\
\left.+\left\langle\left(D_{3}(\varepsilon)+D_{\varepsilon}\left\langle g_{\varepsilon}, e^{-\frac{1}{2} \beta H} g_{1-\varepsilon}\right\rangle\right)^{+} u, G(s)\right\rangle\right)
\end{gathered}
$$

where, $D_{3}(\varepsilon)$ defined by $(6.65)$,

$$
\begin{gathered}
D_{1}(\varepsilon):=D_{\varepsilon}+ \\
+\sum_{n=0}^{\infty} \sum_{\sigma \in\{0,1\}^{n}} D_{\varepsilon} D_{\sigma(1)} \cdots D_{\sigma(n)} D_{1-\varepsilon}
\end{gathered}
$$




$$
\left(g_{1-\varepsilon} \mid g_{\sigma(1)}\right)_{-} \cdot\left(g_{1-\sigma(1)} \mid g_{\sigma(2)}\right)_{-} \cdot \cdots \cdot\left(g_{1-\sigma(n)} \mid g_{1-\varepsilon}\right)_{-}
$$

and

$$
\begin{gathered}
D_{2}(\varepsilon):=\sum_{n=0}^{\infty} \sum_{\sigma \in\{0,1\}^{n}} D_{\varepsilon} D_{\sigma(1)} \cdots \cdot D_{\sigma(n)} D_{\varepsilon} \\
\left(g_{1-\varepsilon} \mid g_{\sigma(1)}\right)_{-} \cdot\left(g_{1-\sigma(1)} \mid g_{\sigma(2)}\right)_{-} \cdots \cdot\left(g_{1-\sigma(n)} \mid g_{\varepsilon}\right)_{-}
\end{gathered}
$$

where, $\sigma(0):=\varepsilon$.

\section{§ 7. The quantum stochastic differential equation}

In the section, we consider the quantum stochastic differential equation (q.s.d.e.)

$$
\begin{gathered}
U(t)=1+\sum_{\varepsilon \in\{0,1\}} \int_{0}^{t}\left[D_{1}(\varepsilon) \otimes d N_{s}\left(g_{\varepsilon}, g_{1-\varepsilon}\right)+\right. \\
\left.+D_{2}(\varepsilon) \otimes d N_{s}\left(g_{\varepsilon}, g_{\varepsilon}\right)+\left(D_{3}(\varepsilon)+D_{\varepsilon}\left\langle g_{1-\varepsilon}, e^{-\frac{1}{2} \beta H} g_{\varepsilon}\right\rangle\right) \otimes 1 d s\right] U(s)
\end{gathered}
$$

on $H_{0} \otimes \Gamma\left(L^{2}(\mathbf{R}) \otimes(\mathrm{K},(\cdot \mid \cdot))\right)$, where, $N$ is the gauge process and

$$
N_{s}\left(g, g^{\prime}\right):=N_{s}\left(\chi_{[0, s]} \otimes|g\rangle\left\langle g^{\prime}\right|\right) .
$$

$D_{1}(\varepsilon), D_{2}(\varepsilon)$ and $D_{3}(\varepsilon)$ are defined in $\S 6$. By [11], we know that the q.s.d.e. (7.1) has a unique solution $U(t)$ which can be obtained by iterative.

Proof of Theorem (1.1). Theorem (6.1) has shown the low density limit (1.13) exists. Now, we shall prove that it is equal to (1.14). Clearly, (1.14) is continuous for $u, v \in H_{0}$, so one can write it to

$$
\langle u, F(t)\rangle
$$

where, $F(t) \in H_{0}$. Hence one has

$$
\begin{aligned}
\langle u, F(0)\rangle=\langle u \otimes W & \left.\left(X_{[S, T]} \otimes f\right) \Psi, v \otimes W\left(\chi_{\left[S^{\prime}, T^{\prime}\right]} \otimes f^{\prime}\right) \Psi\right\rangle \\
& =\langle u, G(0)\rangle .
\end{aligned}
$$

Moreover, by (1.16)

$$
\begin{gathered}
\langle u, F(t)\rangle=\langle u, F(0)\rangle+\sum_{\varepsilon \in\{0,1\}} \int_{0}^{t}\left\langle u \otimes W\left(\chi_{[s, T]} \otimes f^{\prime}\right) \Psi,\right. \\
\left(D_{1}(\varepsilon) \otimes d N_{s}\left(g_{\varepsilon}, g_{1-\varepsilon}\right)+\right. \\
\left.+D_{2}(\varepsilon) \otimes d N_{s}\left(g_{\varepsilon}, g_{\varepsilon}\right)+\left(D_{3}(\varepsilon)+\left\langle g_{1-\varepsilon}, e^{-\frac{1}{2} \beta H} g_{\varepsilon}\right\rangle\right) \otimes 1 d s\right) \\
\left.\times U(s) v \otimes W\left(\chi_{\left[S^{\prime}, T^{\prime}\right]} \otimes f^{\prime}\right) \Psi\right\rangle .
\end{gathered}
$$


Apply the theorem (4.2) of [11] to (7.4), one obtains

$$
\begin{gathered}
\langle u, F(t)\rangle=\langle u, F(0)\rangle+\sum_{\varepsilon \in\{0,1\}} \int_{0}^{t} d s \\
\left(\chi_{[S, T]}(s) \cdot \chi_{\left[S^{\prime}, T^{\prime}\right]}(s) \cdot\left(g_{\varepsilon} \mid f^{\prime}\right) \cdot\left(f \mid g_{\varepsilon}\right)\left\langle D_{1}^{+}(\varepsilon) u, F(s)\right\rangle+\right. \\
+\chi_{[s, T]}(s) \cdot \chi_{\left[S^{\prime}, T^{\prime}\right]}(s) \cdot\left(f \mid g_{\varepsilon}\right)_{-} \cdot\left(g_{1-\varepsilon} \mid f^{\prime}\right) \cdot\left\langle D_{2}^{+}(\varepsilon) u, F(s)\right\rangle+ \\
\left.+\left\langle\left(D_{3}(\varepsilon)+D_{\varepsilon}\left\langle g_{1-\varepsilon}, e^{-\frac{1}{2} \beta H} g_{\varepsilon}\right\rangle\right)^{+} u, F(s)\right\rangle\right) .
\end{gathered}
$$

Since (7.5) has unique solution, so (7.8) has a unique solution. Therefore,

$$
\langle u, F(t)\rangle=\langle u, G(t)\rangle t \geq 0 .
$$

\section{REFERENCES}

[0] L. Accardi, Y. G. Lu, A. Frigerio and R. Alicki, An invitation to the weak coupling limit and the low density limit, to appear in: Quantum probability and related topics VI, World Scientific, 3-31.

[1] L. Accardi, Y. G. Lu, The number process as low density limit of Hamiltonian models, Commun. Math. Phys., 637 (1991), 1-31.

[2] L. Accardi, A. Frigerio, Y. G. Lu, The weak couplihg limit as a functional central limit theorem, Commun. Math. Phys, 131 (1990), 537-570.

[3] L. Accardi, Y. G. Lu, On the low density limit of Boson Models. Lect. Notes in Math, 1442 (1988), 17-53.

[4] - The weak coupling limit for nonlinear interactions, Stoch. Proc. Phys. Geom., World Scientific, 1988, 1-26.

[5] R. Dümcke, The low density limit for $n$-level systems, Lect. Notes in Math, 1136 (1985).

[6] R. Dümcke, The low density limit fot an $N$-level system interacting with a free Bose or fermi gas, Commun. Math. Phys., 97 (1985), 331-359.

[7] A. Frogerio, H. Maassen, Quantum Poisson processes and dilations of dynamical semigroups, Prob. Th. Rel. Fields., 83 (1989), 489-508.

[8] H. Grad, Principles of the knietic theory of Gases, Handbuch der Physik, vol. 12, Springer (1958).

[9] R. L. Hudson, K. R. Pathasarathy, Quantum Ito's formula and stochastic evolution, Commun. Math. Phys., 93 (1984), 301-323.

[10] P. F. Palmer; Thesis, Oxford University.

[11] D. Patz; An invitation to the $C^{*}$-algebra of the canonical commutation relation, Leuven Notes in Math. and Theor. Phys., eds. Fannes M. Verbeure A., Leuven Univ. Press 1990.

Centro Matematico V. Volterra

Dipartimento di Matematica

Universita' di Roma II

Italia 